\title{
THE COHOMOLOGY RINGS OF MODULI SPACES OF BUNDLES OVER RIEMANN SURFACES
}

\author{
FRANCES KIRWAN
}

The cohomology of the moduli space $\mathscr{M}(n, d)$ of semistable bundles of coprime rank $n$ and degree $d$ over a fixed Riemann surface $M$ of genus $g \geq 2$ has been much studied over the last two decades, and a great deal is known about it. It is known that the cohomology has no torsion [2], the Betti numbers have been computed using various different methods $[2,5,11]$, and a set of generators for the cohomology ring is known [2]. The purpose of this article is to provide a method of deciding when an expression in these generators is a relation, and in particular, when the rank $n$ is two, to prove two conjectures about the relations between the generators due to Mumford and to Newstead and Ramanan. Newstead and Ramanan conjectured that certain relations hold among the generators, whereas Mumford conjectured that a certain set of relations is a complete set. In fact the conjecture of Newstead and Ramanan has two parts, the first of which was proved by Gieseker in 1982 [4]. The second part of their conjecture, which is the part examined in this article, was given an independent proof recently by Thaddeus [19], who has a very different method of obtaining information about the relations in the cohomology ring.

The computation of the Betti numbers of the moduli spaces $\mathscr{M}(n, d)$ in [2] stimulated a general method of computing Betti numbers of quotients in the sense of Mumford's geometric invariant theory [14] of nonsingular complex projective varieties by complex reductive group actions (see $[8,9,10]$ ). Similarly the ideas applied in this article to finding generators and relations for the cohomology rings of the moduli spaces $\mathscr{M}(n, d)$ also lead to an analogous method of obtaining generators and relations for the rational cohomology rings of geometric invariant theoretic quotients.

The first section provides background material on the cohomology of the moduli spaces $\mathscr{M}(n, d)$. In the second section, Mumford's conjecture is proved when the rank $n$ is two. The method could, in principle, be used to decide whether the conjecture is true for rank $n>2$ but appears to become significantly more complicated in the general case. The third section gives a method for deciding when an expression in the generators is a relation, and the fourth

Received by the editors July 18, 1991.

1991 Mathematics Subject Classification. Primary 14D20, 32 G13. 
uses this method to prove the Newstead-Ramanan conjecture. The fifth section describes an alternative approach to proving the Newstead-Ramanan conjecture, which involves resolving the singularities of the closures of the strata used by Atiyah and Bott in their calculations of the abelian group structure of the cohomology of the moduli spaces. In the last section the problem of obtaining generators and relations for the cohomology rings of geometric invariant theoretic quotients is discussed, with the actions of $\operatorname{SL}(2 ; \mathbf{C})$ on the spaces of ordered and unordered $n$-tuples of points on the projective line used as examples. This section is independent of the material of earlier sections but is closely analogous to some of it. Many of the arguments exactly parallel those used for the moduli spaces $\mathscr{M}(n, d)$, though there are some crucial differences.

\section{THE COHOMOLOGY OF THE MOdULI SPACES $\mathscr{M}(n, d)$}

Let $\mathscr{M}(n, d)$ be the moduli space of semistable bundles of coprime rank $n$ and degree $d$ over a fixed compact Riemann surface $M$ of genus $g \geq 2$. In [2] Atiyah and Bott describe a construction of $\mathscr{M}(n, d)$ which is closely analogous to the construction of quotients in geometric invariant theory (cf. [14] or $\S 5$ ), but which involves a space $\mathscr{C}$ and a group $\mathscr{G}_{c}$ both of which are infinite dimensional ${ }^{1}$. In this section we shall recall how Atiyah and Bott use this construction to give generators for the cohomology ring of $\mathscr{M}(n, d)$. Our aim is to describe the relations between these generators. In fact it will be enough to describe the relations between generators over the rationals, because Atiyah and Bott show in [2] that the cohomology ring of $\mathscr{M}(n, d)$ is torsion-free.

First recall that a holomorphic vector bundle $E$ over $M$ is called semistable if every holomorphic subbundle $D$ of $E$ satisfies

$$
\mu(D) \leq \mu(E) \quad \text { where } \mu(D)=\frac{\operatorname{degree}(D)}{\operatorname{rank}(D)} .
$$

The bundle $E$ over $M$ is called stable if every proper holomorphic subbundle $D$ of $E$ satisfies

$$
\mu(D)<\mu(E)
$$

When the rank and degree of $E$ are coprime then $E$ is stable if and only if $E$ is semistable.

Let $\mathscr{E}$ be a fixed $C^{\infty}$ complex vector bundle of rank $n$ and degree $d$ over $M$. Then the space $\mathscr{C}$ of all holomorphic structures on $\mathscr{E}$ is an infinitedimensional complex affine space, and the group $\mathscr{G}_{\mathrm{c}}$ of all $C^{\infty}$ complex automorphisms of $\mathscr{E}$ is an infinite-dimensional complex Lie group which acts on $\mathscr{C}$. The moduli space $\mathscr{M}(n, d)$ can be identified with the quotient $\mathscr{C}^{\text {ss }} / \mathscr{G}_{\mathrm{c}}$ of the action of $\mathscr{G}_{\mathrm{c}}$ on the open subset $\mathscr{C}^{\text {ss }}$ of $\mathscr{C}$ consisting of all semistable holomorphic structures on $\mathscr{E}$. The group $\mathscr{G}_{\mathrm{c}}$ is the complexification of the gauge

\footnotetext{
${ }^{1}$ There are alternative constructions of the moduli spaces $\mathscr{M}(n, d)$ as genuine geometric invariant theoretic quotients, which are in some sense finite-dimensional approximations to the construction used by Atiyah and Bott (cf. [11]). All the arguments given in this article could be rephrased in terms of these finite-dimensional quotient constructions.
} 
group $\mathscr{G}$ consisting of all $C^{\infty}$ automorphisms of $\mathscr{E}$ which are unitary with respect to a fixed hermitian structure on $\mathscr{E}$, and there is a natural isomorphism

$$
H^{*}(\mathscr{M}(n, d) ; \mathbf{Z}) \cong H_{\mathscr{G}}^{*}\left(\mathscr{C}^{\text {ss }} ; \mathbf{Z}\right)
$$

where $H_{\mathscr{G}}^{*}$ denotes equivariant cohomology ${ }^{2}$ with respect to the quotient $\overline{\mathscr{G}}$ of $\mathscr{G}$ by its constant central $U(1)$-subgroup which acts trivially on $\mathscr{C}$.

Atiyah and Bott show (see [2, Theorem 7.14]) that the restriction map

$$
H_{\mathscr{G}}^{*}(\mathscr{C} ; \mathbf{Z}) \rightarrow H_{\mathscr{G}}^{*}\left(\mathscr{C}^{s s} ; \mathbf{Z}\right)
$$

is surjective, and since $\mathscr{C}$ is contractible,

$$
H_{\mathscr{G}}^{*}(\mathscr{C} ; \mathbf{Z}) \cong H^{*}(B \overline{\mathscr{G}} ; \mathbf{Z}) \text {. }
$$

Thus generators of the cohomology ring $H^{*}(B \overline{\mathscr{G}} ; \mathbf{Z})$ of the classifying space $B \overline{\mathscr{G}}$ give generators of the cohomology ring of the moduli space $\mathscr{M}(n, d)$. The cohomology rings of the classifying spaces $B \mathscr{G}$ and $B \overline{\mathscr{G}}$ are described in [2, $\S \S 2,9]$. The rational cohomology ring of $B \mathscr{G}$ is freely generated as a graded commutative algebra over the rationals by elements

$$
\begin{gathered}
a_{r} \in H^{2 r}(B \mathscr{G} ; \mathbf{Z}), \\
b_{r}^{j} \in H^{2 r-1}(B \mathscr{G} ; \mathbf{Z})
\end{gathered}
$$

for $1 \leq r \leq n$ and $1 \leq j \leq 2 g$, and

$$
f_{r} \in H^{2 r-2}(B \mathscr{G} ; \mathbf{Z})
$$

for $2 \leq r \leq n$, defined as follows.

The classifying space $B \mathscr{G}$ can be identified with the space $\operatorname{Map}_{d}(M, \mathrm{BU}(n))$ of all $C^{\infty}$ maps from the Riemann surface $M$ to the classifying space of the unitary group $\mathrm{U}(n)$ such that the pullback to $M$ of the classifying bundle on $\mathrm{BU}(n)$ has degree $d$ (see [2, Proposition 2.4]). If we pull back this classifying bundle via the evaluation map

$$
\operatorname{Map}_{d}(M, \mathrm{BU}(n)) \times M \rightarrow \mathrm{BU}(n)
$$

we get a vector bundle $\mathscr{V}$ of rank $n$ over $B \mathscr{G} \times M$. Since $M$ has no torsion, the Künneth formula for the cohomology of a product gives

$$
\begin{aligned}
H^{2 r}(B \mathscr{G} \times M ; \mathbf{Z}) \cong & H^{2 r}(B \mathscr{G} ; \mathbf{Z}) \otimes H^{0}(M ; \mathbf{Z}) \oplus H^{2 r-1}(B \mathscr{G} ; \mathbf{Z}) \\
& \otimes H^{1}(M ; \mathbf{Z}) \oplus H^{2 r-2}(B \mathscr{G} ; \mathbf{Z}) \otimes H^{2}(M ; \mathbf{Z}) .
\end{aligned}
$$

Using this decomposition the $r$ th Chern class of $\mathscr{V}$ can be written as

$$
c_{r}(\mathscr{V})=a_{r} \otimes 1+\sum_{1 \leq j \leq 2 g} b_{r}^{j} \otimes \alpha_{j}+f_{r} \otimes \omega
$$

\footnotetext{
${ }^{2}$ Recall that if a Lie group $G$ acts on a space $X$ then the equivariant cohomology $H_{G}^{*}(X ; \mathbf{Z})$ of $X$ with respect to the action of $G$ is the ordinary cohomology of $X \times{ }_{G} E G$, where $E G \rightarrow B G$ is the universal classifying bundle of $G$.
} 
where $\left\{\alpha_{1}, \ldots, \alpha_{2 g}\right\}$ is a fixed basis for $H^{1}(M ; \mathbf{Z})$ and $\omega$ is the standard generator of $H^{2}(M ; \mathbf{Z})$. This defines the elements $a_{r}, b_{r}^{j}$, and $f_{r}$ which give rational generators for the cohomology ring of $B \mathscr{G}$. The only relations among these generators are that the $a_{r}$ and $f_{r}$ commute with everything and the $b_{r}^{j}$ anticommute with each other.

We have an isomorphism

$$
H^{*}(B \mathscr{G} ; \mathbf{Z}) \cong H^{*}(B \overline{\mathscr{G}} ; \mathbf{Z}) \otimes H^{*}(\mathrm{BU}(1) ; \mathbf{Z})
$$

induced by the fibration $\mathrm{BU}(1) \rightarrow B \mathscr{G} \rightarrow B \overline{\mathscr{G}}$ (see [2, §9]). The rational generators $a_{r}, b_{r}^{j}$, and $f_{r}$ for the cohomology ring of $B \mathscr{G}$ can be pulled back via a section of this fibration to give rational generators of the cohomology ring of $B \overline{\mathscr{G}}$. If we wish we can omit $a_{1}$ since its image in $H^{*}(B \overline{\mathscr{G}} ; \mathbf{Q})$ can be expressed in terms of the other generators. The only relations among the other generators are as above, that the $a_{r}$ and $f_{r}$ commute with everything and the $b_{r}^{j}$ anticommute with each other.

Remark 1. To obtain a set of integral generators for the cohomology rings of $B \mathscr{G}$ and $B \overline{\mathscr{G}}$ we can replace the elements $f_{2}, \ldots, f_{n}$ by the Chern classes $c_{r}\left(\pi_{!}(\mathscr{V})\right)$ for $r \geq 1$ where $\pi: B \mathscr{G} \times M \rightarrow B \mathscr{G}$ is the projection onto the first component and $\pi_{!}$is the direct image map in $K$-theory (see [2, 2.20]).

The conclusion is that when identified with its image under the surjection

$$
H^{*}(B \overline{\mathscr{G}} ; \mathbf{Z}) \cong H_{\mathscr{G}}^{*}(\mathscr{C} ; \mathbf{Z}) \rightarrow H_{\bar{G}}^{*}\left(\mathscr{C}^{\text {ss }} ; \mathbf{Z}\right) \cong H^{*}(\mathscr{M}(n, d) ; \mathbf{Z}),
$$

the set

$$
\left\{a_{r} \mid 2 \leq r \leq n\right\} \cup\left\{b_{r}^{j} \mid 1 \leq r \leq n, 1 \leq j \leq 2 g\right\} \cup\left\{f_{r} \mid 2 \leq r \leq n\right\}
$$

is a set of rational generators for the cohomology ring of the moduli space $\mathscr{M}(n, d)$.

The pullback via the composition

$$
\mathscr{C}^{\text {ss }} \times_{\mathscr{G}} E \mathscr{G} \rightarrow \mathscr{C} \times_{\mathscr{G}} E \mathscr{G} \stackrel{\simeq}{\rightrightarrows} B \mathscr{G}
$$

of the vector bundle $\mathscr{V}$ over $B \mathscr{G} \times M$ induces a $\mathscr{G}$-equivariant holomorphic bundle on $\mathscr{C}^{\text {ss }} \times M$. Since $\overline{\mathscr{G}}$ acts freely on $\mathscr{C}^{\text {ss }}$ with quotient $\mathscr{M}(n, d)$ and the constant central U(1)-subgroup of $\mathscr{G}$ acts as scalar multiplication on the fibres of the pullback of $\mathscr{V}$, the associated projective bundle descends to a holomorphic projective bundle over $\mathscr{M}(n, d) \times M$. In fact there is a holomorphic vector bundle $V$ of rank $n$ and degree $d$ over $\mathscr{M}(n, d) \times M$ whose associated projective bundle is the projective bundle just described (see [2, p. 580]). This bundle is universal in the sense that its restriction to $[E] \times M$, where $[E]$ is the point of the moduli space $\mathscr{M}(n, d)$ represented by a semistable holomorphic bundle $E$ of rank $n$ and degree $d$ over $M$, is isomorphic to the bundle $E$. Generators $a_{r}, b_{r}^{j}$, and $f_{r}$ for the cohomology ring of $\mathscr{M}(n, d)$ can be obtained directly by decomposing the Chern classes of the universal bundle $V$ 
over $\mathscr{M}(n, d) \times M$ according to the Künneth decomposition. To obtain integral generators we take the Chern classes $c_{r}\left(\pi_{!}(V)\right)$, where $\pi$ now denotes the projection of $\mathscr{M}(n, d) \times M$ onto $\mathscr{M}(n, d)$.

The universal bundle $V$ is not unique. We can tensor it by the pullback to $\mathscr{M}(n, d) \times M$ of any holomorphic line bundle $L$ on $\mathscr{M}(n, d)$ and obtain another bundle with the same universal property. This process changes the generators for the cohomology ring of $\mathscr{M}(n, d)$; in particular, $(g-1) a_{1}-$ $c_{1}\left(\pi_{!}(V)\right)$ changes by $c_{1}(L)$. If we take $L=\operatorname{det}\left(\left.V\right|_{\mathscr{M}(n, d)}\right)^{g-1} \otimes\left(\operatorname{det} \pi_{!}(V)\right)^{-1}$ then $c_{1}(L)=(g-1) a_{1}-c_{1}\left(\pi_{!}(V)\right)$ and so $V \otimes \pi^{-1}\left(L^{-1}\right)$ is a new universal bundle such that

$$
(g-1) a_{1}=c_{1}\left(\pi_{!}(V)\right) .
$$

We replace the universal bundle $V$ by this normalised universal bundle (see [2, p. 582]). At the same time we can modify the bundle $\mathscr{V}$ over $B \mathscr{G} \times M$ by the same process and hence still assume that the generators $a_{r}, b_{r}^{j}$, and $f_{r}$ come from generators of $H^{*}(B \mathscr{G} ; \mathbf{Q})$ as above.

We are now in a position to describe the conjectures of Mumford and of Newstead and Ramanan.

Since tensoring by a fixed holomorphic line bundle of degree $e$ gives an isomorphism between the moduli spaces $\mathscr{M}(n, d)$ and $\mathscr{M}(n, d+n e)$, we may assume that

$$
(2 g-2) n<d<(2 g-1) n .
$$

This implies that $H^{1}(M, E)=0$ for any semistable bundle $E$ of rank $n$ and degree $d$ over $M$ and hence that $\pi_{1}(V)$ is the bundle over $\mathscr{M}(n, d)$ with fibre $H^{0}(M, E)$ at $[E]$. The Riemann-Roch theorem then tells us that the rank of this bundle is $d-n(g-1)$. In particular, the Chern classes $c_{r}\left(\pi_{!}(V)\right)$ are zero for $r>d-n(g-1)$. If we express the Chern classes $c_{r}\left(\pi_{!}(\mathscr{V})\right) \in H_{\mathscr{G}}^{*}(\mathscr{C} ; \mathbf{Q})$ in terms of the rational generators $a_{r}, b_{r}^{j}$, and $f_{r}$ of $H_{\mathscr{G}}^{*}(\mathscr{C} ; \mathbf{Q})$, then the fact that their images in $H^{*}(\mathscr{M}(n, d)$; $\mathbf{Q})$ are zero if $r>d-n(g-1)$ gives us relations between the corresponding generators for the cohomology ring of $\mathscr{M}(n, d)$. Mumford conjectured that these relations can be used to obtain a complete set; this conjecture will be stated more precisely and proved in the case when the rank $n$ is two in $\S 2$.

The other conjecture to be discussed in this paper is due to Newstead and Ramanan. They conjectured that when the rank $n$ is two and the degree $d$ is odd, the Pontryagin ring of the tangent bundle to the moduli space $\mathscr{M}(n, d)$ vanishes in dimensions greater than $4(g-1)$. Here the Pontryagin ring is the subring of $H^{*}(\mathscr{M}(n, d) ; \mathbf{Z})$ generated by all the Pontryagin classes of the tangent bundle. A second part of the conjecture is that the $r$ th Chern classes of the moduli space vanish for $r>2(g-1)$; this was proved by Gieseker in [4].

It was shown by Newstead ([16, $\S 4$, Corollary 2 to Theorem 2] or [2, p. 584]) that the Pontryagin ring is actually generated by the first Pontryagin class $p_{1}$. Moreover $p_{1}$ can be expressed in terms of the generators described above for 
the cohomology ring of the moduli space as

$$
p_{1}=2(g-1)\left(a_{1}^{2}-4 a_{2}\right) \text {. }
$$

Thus to prove the conjecture it suffices to show that

$$
\left(a_{1}^{2}-4 a_{2}\right)^{g}=0
$$

in $H^{*}(\mathscr{M}(n, d) ; \mathbf{Q})$. A proof of this will be given in $\S 4$.

The relations between the rational generators $a_{r}, b_{r}^{j}$, and $f_{r}$ are determined by the kernel of the map

$$
H^{*}(B \overline{\mathscr{G}} ; \mathbf{Q}) \cong H_{\mathscr{G}}^{*}(\mathscr{C} ; \mathbf{Q}) \rightarrow H_{\mathscr{G}}^{*}\left(\mathscr{C}^{\text {ss }} ; \mathbf{Q}\right) \cong H^{*}(\mathscr{M}(n, d) ; \mathbf{Q})
$$

which is in turn determined by the map

$$
\begin{aligned}
H_{\mathscr{G}}^{*}(\mathscr{C} ; \mathbf{Q}) & \cong H_{\mathscr{G}}^{*}(\mathscr{C} ; \mathbf{Q}) \otimes H^{*}(\mathrm{BU}(1) ; \mathbf{Q}) \rightarrow H_{\mathscr{G}}^{*}\left(\mathscr{C}^{\mathrm{ss}} ; \mathbf{Q}\right) \\
& \cong H_{\mathscr{G}}^{*}\left(\mathscr{C}^{\mathrm{ss}} ; \mathbf{Q}\right) \otimes H^{*}(\mathrm{BU}(1) ; \mathbf{Q})
\end{aligned}
$$

Our aim is to describe the kernel of this map in terms of the rational generators $a_{r}, b_{r}^{j}$, and $f_{r}$.

To describe this kernel we use the stratification $\left\{\mathscr{C}_{\mu} \mid \mu \in \mathscr{M}\right\}$ of $\mathscr{C}$ described in [2]. This is a stratification indexed by a partially ordered set $\mathscr{M}$ with the following properties.

(i) $\mathscr{C}$ is the disjoint union of the strata, and each stratum $\mathscr{C}_{\mu}$ is a locally closed $\mathscr{G}_{c}$-invariant complex submanifold of finite codimension $d(\mu)$ in $\mathscr{C}$ such that

$$
\overline{\mathscr{C}}_{\mu} \subseteq \bigcup_{\mu^{\prime} \geq \mu} \mathscr{C}_{\mu^{\prime}}
$$

(ii) Given any integer $D$ there are only finitely many $\mu \in \mathscr{M}$ satisfying $d(\mu) \leq D$.

(iii) $\mathscr{C}^{\text {ss }}$ coincides with an open stratum; i.e., there exists some $\mu_{0} \in \mathscr{M}$ such that $\mathscr{C}^{\text {ss }}=\mathscr{C}_{\mu_{0}}$.

(iv) The stratification is equivariantly perfect with respect to the action of the gauge group $\mathscr{G}$ and its quotient $\mathscr{G}_{\mathrm{c}}$, so that there is an isomorphism

$$
H_{\mathscr{G}}^{i}(\mathscr{C} ; \mathbf{Q}) \cong \bigoplus_{\mu \in \mathscr{M}} H_{\mathscr{G}}^{i-2 d(\mu)}\left(\mathscr{C}_{\mu} ; \mathbf{Q}\right)=H_{\mathscr{G}}^{i}\left(\mathscr{C}^{\mathrm{ss}} ; \mathbf{Q}\right) \oplus \bigoplus_{\mu \neq \mu_{0}} H_{\mathscr{G}}^{i-2 d(\mu)}\left(\mathscr{C}_{\mu} ; \mathbf{Q}\right)
$$

for all $i$, where $d(\mu)$ is the complex codimension of $\mathscr{C}_{\mu}$ in $\mathscr{C}$, and the restriction map $H_{\mathscr{G}}^{*}(\mathscr{C} ; \mathbf{Q}) \rightarrow H_{\mathscr{G}}^{*}\left(\mathscr{C}^{\text {ss }} ; \mathbf{Q}\right)$ corresponds under this isomorphism to the projection onto the summand $H_{\mathscr{G}}^{*}\left(\mathscr{C}^{\text {ss }} ; \mathbf{Q}\right)$. The isomorphism is one of vector spaces, not one of rings, and is not canonically determined by the stratification (this will be discussed below).

It follows from this last property of the stratification $\left\{\mathscr{C}_{\mu} \mid \mu \in \mathscr{M}\right\}$ that the kernel of the restriction map $H_{\mathscr{G}}^{*}(\mathscr{C} ; \mathbf{Q}) \rightarrow H_{\mathscr{G}}^{*}\left(\mathscr{C}^{\text {ss }} ; \mathbf{Q}\right)$ is isomorphic as a 
vector space to the direct sum

$$
\bigoplus_{\mu \neq \mu_{0}} H_{\mathscr{G}}^{i-2 d(\mu)}\left(\mathscr{C}_{\mu} ; \mathbf{Q}\right)
$$

(This gave Atiyah and Bott an inductive formula for computing the equivariant Betti numbers of $\mathscr{C}^{\text {ss }}$ and thus the Betti numbers of the moduli space.) However, our aim is to describe the kernel as a subset of $H_{\mathscr{G}}^{*}(\mathscr{C} ; \mathbf{Q})$. If $\mu \in \mathscr{M}$ then by property (i) of the stratification, the subset

$$
U_{\mu}=\mathscr{C}-\bigcup_{\nu>\mu} \mathscr{C}_{\nu}=\bigcup_{\nu \ngtr \mu} \mathscr{C}_{\nu}
$$

is an open subset of $\mathscr{C}$ containing $\mathscr{C}_{\mu}$ as a closed submanifold. Thus there is a long exact sequence (the Thom-Gysin sequence)

$$
\cdots \rightarrow H_{\mathscr{G}}^{j-2 d(\mu)}\left(\mathscr{C}_{\mu} ; \mathbf{Q}\right) \rightarrow H_{\mathscr{G}}^{j}\left(U_{\mu} ; \mathbf{Q}\right) \rightarrow H_{\mathscr{G}}^{j}\left(U_{\mu}-\mathscr{C}_{\mu} ; \mathbf{Q}\right) \rightarrow \cdots
$$

To say that the stratification is equivariantly perfect over the rationals ${ }^{3}$ means that all these long exact sequences break up into short exact sequences

$$
0 \rightarrow H_{\mathscr{G}}^{j-2 d(\mu)}\left(\mathscr{C}_{\mu} ; \mathbf{Q}\right) \rightarrow H_{\mathscr{G}}^{j}\left(U_{\mu} ; \mathbf{Q}\right) \rightarrow H_{\mathscr{G}}^{j}\left(U_{\mu}-\mathscr{C}_{\mu} ; \mathbf{Q}\right) \rightarrow 0 .
$$

The fact that the stratification is equivariantly perfect thus implies that the restriction maps $H_{\mathscr{G}}^{*}(\mathscr{C} ; \mathbf{Q}) \rightarrow H_{\mathscr{G}}^{*}\left(U_{\mu} ; \mathbf{Q}\right)$ are all surjective, and hence the Thom-Gysin maps $H_{\mathscr{G}}^{j-2 d(\mu)}\left(\mathscr{C}_{\mu} ; \mathbf{Q}\right) \rightarrow H_{\mathscr{G}}^{j}\left(U_{\mu} ; \mathbf{Q}\right)$ can be lifted to maps $H_{\mathscr{G}}^{j-2 d(\mu)}\left(\mathscr{C}_{\mu} ; \mathbf{Q}\right) \rightarrow H_{\mathscr{G}}^{j}(\mathscr{C} ; \mathbf{Q})$.

Lemma 1. The direct sum

$$
\bigoplus_{\mu \neq \mu_{0}} H_{\mathscr{G}}^{j-2 d(\mu)}\left(\mathscr{C}_{\mu} ; \mathbf{Q}\right) \rightarrow H_{\mathscr{G}}^{j}(\mathscr{C} ; \mathbf{Q})
$$

of any choice of lifts $H_{\mathscr{G}}^{j-2 d(\mu)}\left(\mathscr{C}_{\mu} ; \mathbf{Q}\right) \rightarrow H_{\mathscr{G}}^{j}(\mathscr{C} ; \mathbf{Q})$ of the Thom-Gysin maps $H_{\mathscr{G}}^{j-2 d(\mu)}\left(\mathscr{C}_{\mu} ; \mathbf{Q}\right) \rightarrow H_{\mathscr{G}}^{j}\left(U_{\mu} ; \mathbf{Q}\right)$ is an isomorphism onto its image, which is the kernel of the restriction map $H_{\mathscr{G}}^{j}(\mathscr{C} ; \mathbf{Q}) \rightarrow H_{\mathscr{G}}^{j}\left(\mathscr{C}^{\text {ss }} ; \mathbf{Q}\right)$.

The proof is immediate.

The stratification is defined as follows. Any holomorphic bundle $E$ over $M$ of rank $n$ and degree $d$ has a canonical filtration

$$
0=E_{0} \subset E_{1} \subset \cdots \subset E_{s}=E
$$

such that the quotient bundles $D_{j}=E_{j} / E_{j-1}$ are semistable, and their ranks $n_{j}$ and degree $d_{j}$ satisfy

$$
d_{j} / n_{j}>d_{j+1} / n_{j+1} \quad \text { if } 1 \leq j<s
$$

\footnotetext{
${ }^{3}$ In fact the stratification is equivariantly perfect over the integers, i.e., the Thom-Gysin sequences of integer cohomology break up into short exact sequences, but we shall not use this directly.
} 
The type $\mu$ of $E$ is defined to be the vector

$$
\mu=\left(d_{1} / n_{1}, \ldots, d_{s} / n_{s}\right) \in \mathbf{Q}^{n}
$$

in which each $d_{j} / n_{j}=\mu\left(D_{j}\right)$ occurs successively $n_{j}$ times in increasing order of $j$. The bundle $E$ is then semistable if and only if it is of type $\mu_{0}$ where

$$
\mu_{0}=(d / n, \ldots, d / n) .
$$

The indexing set $\mathscr{M}$ is the set of all possible types of holomorphic bundles of rank $n$ and degree $d$. If $\mu=\left(\mu_{1}, \ldots, \mu_{n}\right)$ and $\lambda=\left(\lambda_{1}, \ldots, \lambda_{n}\right)$ belong to $\mathscr{M}$ then we say $\lambda \geq \mu$ if $\sum_{j \leq i} \lambda_{j} \geq \sum_{j \leq i} \mu_{j}$ whenever $1 \leq j<n$; this defines a partial order on $\mathscr{M}$ (see $[2,7.7])$. The stratum $\mathscr{C}_{\mu}$ of $\mathscr{C}$ indexed by $\mu \in \mathscr{M}$ is the set of all holomorphic structures on the fixed $C^{\infty}$ bundle $\mathscr{E}$ of type $\mu$.

Atiyah and Bott show that the stratification is equivariantly perfect by showing first of all that if

$$
\mu=\left(d_{1} / n_{1}, \ldots, d_{s} / n_{s}\right) \in \mathscr{M}
$$

then

$$
H_{\mathscr{G}}^{*}\left(\mathscr{C}_{\mu} ; \mathbf{Q}\right) \cong \bigotimes_{1 \leq i \leq s} H_{\mathscr{G}\left(n_{i}, d_{i}\right)}^{*}\left(\mathscr{C}\left(n_{i}, d_{i}\right)^{\text {ss }} ; \mathbf{Q}\right)
$$

where $\mathscr{C}\left(n_{i}, d_{i}\right)$ is the space of all holomorphic structures on a fixed hermitian vector bundle of rank $n_{i}$ and degree $d_{i}$ over $M$, and $\mathscr{G}\left(n_{i}, d_{i}\right)$ is the gauge group of that bundle [2,7.12]. They then show that the image under this isomorphism of the equivariant Euler class of the normal bundle to the stratum $\mathscr{C}_{\mu}$ in $\mathscr{C}$ is not a zero divisor in $\bigotimes_{1 \leq i \leq s} H_{\mathscr{G}\left(n_{i}, d_{i}\right)}^{*}\left(\mathscr{C}\left(n_{i}, d_{i}\right)^{\text {ss }} ; \mathbf{Q}\right)$ [2, p. 569]. This implies that the composition of the Thom-Gysin map $H_{\mathscr{G}}^{j-2 d(\mu)}\left(\mathscr{C}_{\mu} ; \mathbf{Q}\right) \rightarrow$ $H_{\mathscr{G}}^{j}\left(U_{\mu} ; \mathbf{Q}\right)$ with the restriction map

$$
H_{\mathscr{G}}^{j}\left(U_{\mu} ; \mathbf{Q}\right) \rightarrow H_{\mathscr{G}}^{j}\left(\mathscr{C}_{\mu} ; \mathbf{Q}\right)
$$

is injective (the composition is just multiplication by the equivariant Euler class of the normal bundle), and hence that the Thom-Gysin maps themselves are injective.

We shall need to calculate the equivariant Euler class of the normal bundle to the stratum $\mathscr{C}_{\mu}$ in $\mathscr{C}$ in terms of the set of generators

$$
\bigcup_{1 \leq i \leq s}\left(\left\{a_{r}^{i} \mid 1 \leq r \leq n_{i}\right\} \cup\left\{b_{r}^{i, j} \mid 1 \leq r \leq n_{i}, 1 \leq j \leq 2 g\right\} \cup\left\{f_{r}^{i} \mid 2 \leq r \leq n_{i}\right\}\right)
$$

for $\bigotimes_{1 \leq i \leq s} H_{\mathscr{G}\left(n_{i}, d_{i}\right)}^{*}\left(\mathscr{C}\left(n_{i}, d_{i}\right)^{\text {ss }} ; \mathbf{Q}\right)$ corresponding to the generators for $H_{\mathscr{G}}^{*}(\mathscr{C} ; \mathbf{Q})$ defined as above. The important case for our purposes is when $n_{i}=1$ for each $i$.

Lemma 2. Suppose that $\mu=\left(d_{1}, \ldots, d_{n}\right) \in \mathscr{M}$ where $d_{1}>d_{2}>\cdots>d_{n}$. Then the equivariant Euler class

$$
e_{\mu} \in \bigotimes_{1 \leq i \leq n} H_{\mathscr{G}\left(1, d_{i}\right)}^{*}\left(\mathscr{C}\left(1, d_{i}\right)^{\text {ss }} ; \mathbf{Q}\right)
$$


of the normal bundle in $\mathscr{C}$ to the stratum $\mathscr{C}_{\mu}$ can be expressed in terms of the generators

$$
\begin{gathered}
\left\{a_{1}^{i} \mid 1 \leq i \leq n\right\} \cup\left\{b_{1}^{i, j} \mid 1 \leq i \leq n, 1 \leq j \leq 2 g\right\} \\
\text { of } \left.\otimes_{1 \leq i \leq n} H_{\mathscr{G}_{\left(1, d_{i}\right)}\left(\mathscr{C}\left(1, d_{i}\right)^{\mathrm{ss}}\right.} ; \mathbf{Q}\right) \text { as } \\
\prod_{j>i}\left(a_{1}^{j}-a_{1}^{i}\right)^{d_{i}-d_{j}-1}\left(\left(a_{1}^{j}-a_{1}^{i}\right)^{g}-\xi^{i, j}\left(a_{1}^{j}-a_{1}^{i}\right)^{g-1}\right. \\
\left.+\frac{\left(\xi^{i, j}\right)^{2}}{2 !}\left(a_{1}^{j}-a_{1}^{i}\right)^{g-2}+\cdots+\frac{\left(-\xi^{i, j}\right)^{g}}{g !}\right)
\end{gathered}
$$

where

$$
\xi^{i, j}=\sum_{1 \leq k \leq g}\left(b_{1}^{j, k}-b_{1}^{i, k}\right)\left(b_{1}^{j, k+g}-b_{1}^{i, k+g}\right) .
$$

Proof. The fibre at $E \in \mathscr{C}_{\mu}$ of the normal bundle to the stratum $\mathscr{C}_{\mu}$ in $\mathscr{C}$ can be identified in a natural way with

$$
H^{1}\left(M, \text { End }^{\prime \prime} E\right)
$$

where End $E$ is the bundle of holomorphic endomorphisms of $E$ and

$$
\text { End }^{\prime \prime} E=\text { End } E / \text { End }^{\prime} E
$$

is the quotient of End $E$ by its subbundle consisting of the endomorphisms which preserve the canonical filtration

$$
0=E_{0} \subset E_{1} \subset \cdots \subset E_{s}=E
$$

(see [2, p. 566]). The isomorphism

$$
\bigotimes_{1 \leq i \leq s} H_{\mathscr{G}\left(n_{i}, d_{i}\right)}^{*}\left(\mathscr{C}\left(n_{i}, d_{i}\right)^{\mathrm{ss}} ; \mathbf{Q}\right) \cong H_{\mathscr{G}}^{*}\left(\mathscr{C}_{\mu} ; \mathbf{Q}\right)
$$

used by Atiyah and Bott is induced by the map

$$
\prod_{1 \leq i \leq s} \mathscr{C}\left(n_{i}, d_{i}\right)^{\mathrm{ss}} \rightarrow \mathscr{C}_{\mu}
$$

that sends a sequence of semistable bundles $\left(D_{1}, \ldots, D_{s}\right)$ to the direct sum $D_{1} \oplus \cdots \oplus D_{s}$. If $E$ is of the form $D_{1} \oplus \cdots \oplus D_{s}$ then the bundle End" $E$ is $\bigoplus_{i<j} D_{i}^{*} \otimes D_{j}$, so

$$
H^{1}\left(M, \operatorname{End}^{\prime \prime} E\right)=\bigoplus_{i<j} H^{1}\left(M, D_{i}^{*} \otimes D_{j}\right) .
$$

Moreover, $H^{0}\left(M\right.$, End $\left.^{\prime \prime} E\right)=0$, so in terms of $K$-theory the normal bundle to the stratum $\mathscr{C}_{\mu}$ in $\mathscr{C}$ is

$$
-\pi_{!}\left(\bigoplus_{i<j} \mathscr{V}_{i}^{*} \otimes \mathscr{V}_{j}\right)
$$


where $\mathscr{V}_{i}$ is the universal bundle on $\mathscr{C}\left(n_{i}, d_{i}\right)$ and $\pi: \prod_{1 \leq i \leq s} \mathscr{C}\left(n_{i}, d_{i}\right)^{\mathrm{ss}} \times$ $M \rightarrow \prod_{1 \leq i \leq s} \mathscr{C}\left(n_{i}, d_{i}\right)^{\text {ss }}$ is the projection on the first component. In the case under consideration, $s=n$ and the universal bundles $\mathscr{V}_{1}, \ldots, \mathscr{V}_{n}$ are all line bundles.

We want to find the equivariant Euler class (i.e., the equivariant top Chern class) of $-\pi_{!}\left(\mathscr{V}_{i}^{*} \otimes \mathscr{V}_{j}\right)$ when $i<j$. If we let ch denote the equivariant Chern character then by the Riemann-Roch theorem

$$
\operatorname{ch}\left(\pi_{!}\left(\mathscr{V}_{i}^{*} \otimes \mathscr{V}_{j}\right)\right)=\pi^{*}\left\{\operatorname{ch}\left(\mathscr{V}_{i}^{*} \otimes \mathscr{V}_{j}\right)(1-\bar{g} \omega)\right\}
$$

where $\bar{g}=g-1$ and $\omega$ is the natural generator of $H^{2}(M, \mathbf{Z})$. In terms of the generators

$$
\left\{a_{1}^{i} \mid 1 \leq i \leq n\right\} \cup\left\{b_{1}^{i, j} \mid 1 \leq i \leq n, 1 \leq j \leq 2 g\right\}
$$

of $\bigotimes_{1 \leq i \leq n} H_{\mathscr{G}\left(1, d_{i}\right)}^{*}\left(\mathscr{C}\left(1, d_{i}\right)^{\text {ss }} ; \mathbf{Q}\right)$ we have

$$
\operatorname{ch}\left(\mathscr{V}_{i}\right)=e^{a_{1}^{i}+\sum_{k=1}^{2 g} b_{1}^{i, k} \otimes \alpha_{k}+d_{i} \omega} .
$$

Therefore

$$
\operatorname{ch}\left(\mathscr{V}_{i}^{*} \otimes \mathscr{V}_{j}\right)=e^{a_{1}^{j}-a_{1}^{i}}\left(1+\sum_{1 \leq k \leq 2 g}\left(b_{1}^{j, k}-b_{1}^{i, k}\right) \otimes \alpha_{k}+\left(\xi^{i, j}+d_{j}-d_{i}\right) \omega\right),
$$

so

$$
\pi^{*}\left(\operatorname{ch}\left(\mathscr{V}_{i}^{*} \otimes \mathscr{V}_{j}\right)(1-\bar{g} \omega)\right)=e^{a_{1}^{j}-a_{1}^{i}}\left(\xi^{i, j}+d_{j}-d_{i}-\bar{g}\right)
$$

Thus

$$
\operatorname{ch}\left(-\pi_{!}\left(\bigoplus_{j>i} \mathscr{V}_{i}^{*} \otimes \mathscr{V}_{j}\right)\right)=\sum_{j>i} e^{a_{1}^{j}-a_{1}^{i}}\left(d_{i}-d_{j}+\bar{g}-\xi^{i, j}\right) .
$$

Now let $\lambda_{1}, \ldots, \lambda_{g}$ be the roots of the equation

$$
x^{g}-x^{g-1}+\frac{1}{2} x^{g-2}-\cdots+\frac{(-1)^{j}}{j !} x^{g-j}+\cdots+\frac{(-1)^{g}}{g !}=0 .
$$

Let $s_{r}=\sum_{1 \leq k \leq g} \lambda_{k}^{r}$ and let $\sigma_{r}$ be the $r$ th elementary symmetric polynomial in $\lambda_{1}, \ldots, \lambda_{g}$. Then $\sigma_{r}=1 / r$ ! , and so from Newton's formula (see [13, p. 195])

$$
s_{r}=(-1)^{r+1} r \sigma_{r}+(-1)^{r} \sigma_{r-1} s_{1}+\cdots-\sigma_{2} s_{r-2}+\sigma_{1} s_{r-1}
$$

we obtain

$$
\begin{aligned}
& s_{1}=\sigma_{1}=1, \\
& s_{2}=1-1=0,
\end{aligned}
$$

and by induction

$$
s_{r}=\left((-1)^{r+1} r / r !\right)+\left((-1)^{r} /(r-1) !\right)=0
$$


for $2 \leq r \leq g$. Since $\left(\xi^{i, j}\right)^{g+1}=0$ for each $i$ and $j$, we have

$$
\sum_{1 \leq k \leq g} e^{-\lambda_{k} \xi^{i, j}}=g-\xi^{i, j}
$$

and hence

$$
\operatorname{ch}\left(-\pi_{!}\left(\bigoplus_{j>1} \mathscr{V}_{i}^{*} \otimes \mathscr{V}_{j}\right)\right)=\sum_{j>i}\left(\sum_{1 \leq k \leq g} e^{-\lambda_{k} \xi^{i, j}+a_{1}^{j}-a_{1}^{i}}\right)+\left(d_{i}-d_{j}-1\right) e^{a_{1}^{j}-a_{1}^{i}}
$$

Since $d_{i}>d_{j}$ when $j>i$, this means that the Euler class $e_{\mu}$ is given by

$$
e_{\mu}=\prod_{j>i}\left(a_{1}^{j}-a_{1}^{i}\right)^{d_{i}-d_{j}-1} \prod_{1 \leq k \leq g}\left(-\lambda_{k} \xi^{i, j}+a_{1}^{j}-a_{1}^{i}\right)
$$

or equivalently by

$$
e_{\mu}=\prod_{j>i}\left(a_{1}^{j}-a_{1}^{i}\right)^{d_{i}-d_{j}-1}\left(\left(a_{1}^{j}-a_{1}^{i}\right)^{g}-\xi^{i, j}\left(a_{1}^{j}-a_{1}^{i}\right)^{g-1}+\cdots+\frac{\left(-\xi^{i, j}\right)^{g}}{g !}\right)
$$

as required.

Remark 2. Now suppose more generally that $\mu=\left(d_{1} / n_{1}, \ldots, d_{s} / n_{s}\right)$ is an element of $\mathscr{M}$ not necessarily satisfying $n_{j}=1$ for all $j$, and let

$$
e_{\mu} \in H_{\mathscr{G}}^{*}\left(\mathscr{C}_{\mu} ; \mathbf{Q}\right) \cong \bigotimes_{1 \leq i \leq s} H_{\mathscr{G}\left(n_{i}, d_{i}\right)}^{*}\left(\mathscr{C}\left(n_{i}, d_{i}\right)^{\text {ss }} ; \mathbf{Q}\right)
$$

be the equivariant Euler class of the normal bundle to the stratum $\mathscr{C}_{\mu}$ in $\mathscr{C}$. It was shown in the proof of Lemma 2 that in terms of $K$-theory this normal bundle is

$$
-\pi_{!}\left(\bigoplus_{i<j} \mathscr{V}_{i}^{*} \otimes \mathscr{V}_{j}\right)
$$

where $\mathscr{V}_{i}$ is the universal bundle on $\mathscr{C}\left(n_{i}, d_{i}\right)$ and $\pi: \prod_{1 \leq i \leq s} \mathscr{C}\left(n_{i}, d_{i}\right)^{\mathrm{ss}} \times$ $M \rightarrow \prod_{1 \leq i \leq s} \mathscr{C}\left(n_{i}, d_{i}\right)^{\text {ss }}$ is the projection on the first component. In order to calculate the Euler class $e_{\mu}$ we formally write

$$
\operatorname{ch}\left(\mathscr{V}_{i}\right)=\sum_{1 \leq \ell \leq n_{i}} e^{a_{1}^{i, \ell}+\sum_{k=1}^{2 g} b_{1}^{i, \ell, k} \otimes \alpha_{k}+d^{i, \ell} \omega}
$$

where the $a_{1}^{i, \ell}, b_{1}^{i, \ell, k}$, and $d^{i, \ell}$ are indeterminates such that the $r$ th Chern class

$$
c_{r}\left(\mathscr{V}_{i}\right)=a_{r}^{i}+\sum_{1 \leq j \leq 2 g} b_{r}^{i, j} \otimes \alpha_{j}+f_{r} \omega
$$

of $\mathscr{V}_{i}$ is given by the $r$ th elementary symmetric polynomial in the expressions

$$
a_{1}^{i, \ell}+\sum_{k=1}^{2 g} b_{1}^{i, \ell, k} \otimes \alpha_{k}+d^{i, \ell} \omega
$$


when $1 \leq r \leq n_{i}$. Using the Riemann-Roch theorem as in the proof of Lemma 2 , it follows that the Chern character of the normal bundle to the stratum $\mathscr{C}_{\mu}$ in $\mathscr{C}$ is given by

$$
\sum_{j>i} \sum_{1 \leq \ell \leq n_{i}} \sum_{1 \leq m \leq n_{j}} e^{a_{1}^{j, m}-a_{1}^{i, \ell}}\left(d^{i, \ell}-c^{j, m}+\bar{g}-\xi_{\ell, m}^{i, j}\right)
$$

where

$$
\xi_{\ell, m}^{i, j}=\sum_{1 \leq k \leq g}\left(b_{1}^{j, m, k}-b_{1}^{i, \ell, k}\right)\left(b_{1}^{j, m, k+g}-b_{1}^{i, \ell, k+g}\right) .
$$

Formally we can write this Chern character as $\sum_{1 \leq q \leq d(\mu)} e^{h_{q}}$ where

$$
d(\mu)=\sum_{1 \leq i<j \leq s} d_{j} n_{i}-d_{i} n_{j}+n_{i} n_{j} \bar{g}
$$

is the rank of the normal bundle to the stratum $\mathscr{C}_{\mu}$ in $\mathscr{C}$ and

$$
\begin{aligned}
\sum_{1 \leq q \leq d(\mu)}\left(h_{q}\right)^{r}= & \sum_{j>i} \sum_{1 \leq \ell \leq n_{i}} \sum_{1 \leq m \leq n_{j}}\left(a_{1}^{j, m}-a_{1}^{i, \ell}\right)^{r}\left(d^{i, \ell}-c^{j, m}+\bar{g}-\xi_{\ell, m}^{i, j}\right) \\
& -r \xi_{\ell, m}^{i, j}\left(a_{1}^{j, m}-a_{1}^{i, \ell}\right)^{r-1}
\end{aligned}
$$

when $1 \leq r \leq d(\mu)$. The Euler class $e_{\mu}=\prod_{1 \leq q \leq d(\mu)} h_{q}$ can then be expressed, by using the general identities relating elementary symmetric polynomials and sums of powers given in the proof of Lemma 2 , in terms of the sums

$\sum_{j>i} \sum_{1 \leq \ell \leq n_{i}} \sum_{1 \leq m \leq n_{j}}\left(a_{1}^{j, m}-a_{1}^{i, \ell}\right)^{r}\left(d^{i, \ell}-c^{j, m}+\bar{g}+\xi_{\ell, m}^{i, j}\right)+r \xi_{\ell, m}^{i, j}\left(a_{1}^{j, m}-a_{1}^{i, \ell}\right)^{r-1}$,

which can in turn be expressed in terms of the generators $a_{r}^{i}, b_{r}^{i, j}$, and $f_{r}$ of $H_{\mathscr{G}\left(n_{i}, d_{i}\right)}^{*}\left(\mathscr{C}\left(n_{i}, d_{i}\right)^{s s} ; \mathbf{Q}\right)$.

\section{Proof of Mumford's conjecture}

In this section we shall prove Mumford's conjecture in the case when the rank $n$ is two. Mumford conjectured that a certain set of relations among the generators for the cohomology ring $H^{*}(\mathscr{M}(n, d)$; $\mathbf{Q})$ described in the last section is complete. First we must give a precise description of this set of relations.

We may assume as in the last section without any loss of generality that

$$
(2 g-2) n<d<(2 g-1) n .
$$

Since $d$ and $n$ are coprime and $n=2$ this forces the degree to be $d=4 g-3$. We noted that then $H^{1}(M, E)=0$ for any semistable bundle $E$ of rank 2 and degree $d$ over $M$. This means that if $V$ is the normalised universal bundle over $\mathscr{M}(2, d) \times M$ described in the last section and $\pi: \mathscr{M}(2, d) \times M \rightarrow$ $\mathscr{M}(2, d)$ is the projection onto the first component then $\pi_{!}(V)$ is a bundle 
over $\mathscr{M}(2, d)$ with fibre $H^{0}(M, E)$ at $[E]$. By the Riemann-Roch theorem the rank of this bundle is $d-2(g-1)=2 g-1$. In particular, the Chern classes $c_{r}\left(\pi_{!}(V)\right)$ are zero for $r \geq 2 g$. If we express the Chern classes $c_{r}\left(\pi_{1}(\mathscr{V})\right) \in H_{\mathscr{G}}^{*}(\mathscr{C} ; \mathbf{Q})$ in terms of the rational generators $a_{r}, b_{r}^{j}$, and $f_{r}$ of $H_{\mathscr{G}}^{*}(\mathscr{C} ; \mathbf{Q})$, then the fact that their images in $H^{*}(\mathscr{M}(n, d) ; \mathbf{Q})$ are zero if $r \geq 2 g$ gives us relations between the corresponding generators for the cohomology ring of $\mathscr{M}(n, d)$. But we can get more information if we recall from $[2, \S 9]$ that the cohomology ring $H^{*}(\mathscr{M}(2, d) ; \mathbf{Q})$ is isomorphic to the tensor product of the cohomology ring of the Jacobian of the Riemann surface $M$ with the cohomology ring $H^{*}\left(\mathscr{M}_{0}(2, d) ; \mathbf{Q}\right)$ of the moduli space $\mathscr{M}_{0}(2, d)$ of bundles of rank 2 and degree $d$ and fixed determinant line bundle. The cohomology ring $H_{\mathscr{G}}^{*}(\mathscr{C} ; \mathbf{Q})$ is freely generated as a graded commutative $\mathbf{Q}$ algebra by the elements $a_{r}, b_{r}^{j}$, and $f_{r}$. The cohomology ring of the Jacobian is an exterior algebra on $2 g$ generators, and the generators can be chosen so that under this isomorphism they correspond to the elements $b_{1}^{1}, \ldots, b_{1}^{2 g}$ of $H^{*}(\mathscr{M}(2, d) ; \mathbf{Q})$, while the elements $a_{1}, a_{2}, b_{2}^{j}$, and $f_{2}$ correspond to generators of $H^{*}\left(\mathscr{M}_{0}(2, d) ; \mathbf{Q}\right)$. Therefore we can obtain relations among the images in the cohomology ring $H^{*}(\mathscr{M}(2, d) ; \mathbf{Q})$ of the rational generators $a_{1}, a_{2}, b_{1}^{j}, b_{2}^{j}$, and $f_{2}$ of $H_{\mathscr{G}}^{*}(\mathscr{C} ; \mathbf{Q})$ if we express the Chern classes $c_{r}\left(\pi_{\dot{1}}(\mathscr{V})\right) \in H_{\mathscr{G}}^{*}(\mathscr{C} ; \mathbf{Q})$ for $r \geq 2 g$ in terms of these generators and then equate to zero the coefficients (in the obvious sense) of $\prod_{j \in S} b_{1}^{j}$ for any subset $S$ of $\{1, \ldots, 2 g\}$. Of course we could equivalently express the Chern classes $c_{r}\left(\pi_{!}(\mathscr{V})\right) \in H_{\mathscr{G}}^{*}(\mathscr{C} ; \mathbf{Q})$ for $r \geq 2 g$ in terms of $a_{1}, a_{2}, b_{1}^{j}$,

$$
2 b_{2}^{j}-a_{1} b_{1}^{j}, \quad \text { and } \quad 2 f_{2}-\sum_{j=1}^{g} b_{1}^{j} b_{1}^{j+g}
$$

and again equate to zero the coefficients of $\prod_{j \in S} b_{1}^{j}$ for any subset $S$ of $\{1, \ldots, 2 g\}$; this will be more convenient. The reason is that if $\delta \in \mathscr{M}-\left\{\mu_{0}\right\}$ the restriction map

$$
H_{\mathscr{G}}^{*}(\mathscr{C} ; \mathbf{Q}) \rightarrow H_{\mathscr{G}}^{*}\left(\mathscr{C}_{\delta} ; \mathbf{Q}\right)
$$

is given by

$$
\begin{gathered}
a_{1} \mapsto a_{1}^{1}+a_{1}^{2}, \quad a_{2} \mapsto a_{1}^{1} a_{1}^{2} \\
b_{1}^{j} \mapsto b_{1}^{1, j}+b_{1}^{2, j}, \quad b_{2}^{j} \mapsto a_{1}^{1} b_{1}^{2, j}+a_{1}^{2} b_{1}^{1, j},
\end{gathered}
$$

and

$$
f_{2} \mapsto \delta_{2} a_{1}^{1}+\delta_{1} a_{1}^{2}+\sum_{j=1}^{g} b_{1}^{1, j} b_{1}^{2, j+g}+b_{1}^{2, j} b_{1}^{1, j+g} .
$$

This means that the restrictions of $a_{1}, a_{2}, 2 b_{2}^{j}-a_{1} b_{1}^{j}$, and $2 f_{2}-\sum_{j=1}^{g} b_{1}^{j} b_{1}^{j+g}$ can all be expressed in terms of $a_{1}^{1}, a_{1}^{2}$, and $b_{1}^{1, j}-b_{1}^{2, j}$. Thus given the restriction $x_{\delta}$ to $H_{\mathscr{G}}^{*}\left(\mathscr{C}_{\delta} ; \mathbf{Q}\right)$ of $x \in H_{\mathscr{G}}^{*}(\mathscr{C} ; \mathbf{Q})$, it is easy to work out the restrictions of the coefficients of $\prod_{j \in S} b_{1}^{j}$ in $x$ for any subset $S$ of $\{1, \ldots, 2 g\}$; simply 
take the coefficients of $\prod_{j \in S}\left(b_{1}^{1, j}+b_{1}^{2, j}\right)$ in the unique expression for $x_{\delta}$ in terms of $a_{1}^{1}, a_{1}^{2}, b_{1}^{1, j}+b_{1}^{2, j}$, and $b_{1}^{1, j}-b_{1}^{2, j}$.

We shall prove Mumford's conjecture in the following form.

Theorem 1. The set $\mathscr{R}$ of relations obtained in this way, together with the relation $(g-1) a_{1}=c_{1}\left(\pi_{!}(\mathscr{V})\right)$ due to the normalisation of the universal bundle $V$, is a complete set of relations among the rational generators $a_{1}, a_{2}, b_{1}^{j}, b_{2}^{j}$, and $f_{2}$ of $H^{*}(\mathscr{M}(2, d) ; \mathbf{Q})$.

In fact we shall prove an equivalent statement, which is that the ideal of the equivariant cohomology ring

$$
H_{\mathscr{G}}^{*}(\mathscr{C} ; \mathbf{Q}) \cong H^{*}(B \mathscr{G} ; \mathbf{Q})
$$

generated by $\mathscr{R}$ is the kernel of the restriction map

$$
H_{\mathscr{G}}^{*}(\mathscr{C} ; \mathbf{Q}) \rightarrow H_{\mathscr{G}}^{*}\left(\mathscr{C}^{\mathrm{ss}} ; \mathbf{Q}\right) \text {. }
$$

We know that $\mathscr{R}$ is contained in the kernel; therefore, we shall be able to use the following proposition.

Recall that if $\mu \in \mathscr{M}$ then $e_{\mu} \in H_{\mathscr{G}}^{*}\left(\mathscr{C}_{\mu} ; \mathbf{Q}\right)$ is the equivariant Euler class of the normal bundle to the stratum $\mathscr{C}_{\mu}$ in $\mathscr{C}$. Recall also that the stratum indexed by $\mu_{0}=(d / n, \ldots, d / n)$ is the semistable stratum $\mathscr{C}^{\text {ss }}$.

Proposition 1. Let $\mathscr{R}$ be a subset of the kernel of the restriction map

$$
H_{\mathscr{G}}^{*}(\mathscr{C} ; \mathbf{Q}) \rightarrow H_{\mathscr{G}}^{*}\left(\mathscr{C}^{\mathrm{ss}} ; \mathbf{Q}\right) \text {. }
$$

Suppose that for each $\mu \in \mathscr{M}-\left\{\mu_{0}\right\}$ there is a subset $\mathscr{R}_{\mu}$ of $\mathscr{R}$ such that the image of the ideal generated by $\mathscr{R}_{\mu}$ under the restriction map

$$
H_{\mathscr{G}}^{*}(\mathscr{C} ; \mathbf{Q}) \rightarrow H_{\mathscr{G}}^{*}\left(\mathscr{C}_{\tilde{\mu}} ; \mathbf{Q}\right)
$$

is zero unless $\tilde{\mu} \geq \mu$ and is the ideal of $H_{\mathscr{G}}^{*}\left(\mathscr{C}_{\mu} ; \mathbf{Q}\right)$ generated by the Euler class $e_{\mu}$ if $\tilde{\mu}=\mu$. Then $\mathscr{R}$ generates the kernel of the restriction map

$$
H_{\mathscr{G}}^{*}(\mathscr{C} ; \mathbf{Q}) \rightarrow H_{\mathscr{G}}^{*}\left(\mathscr{C}^{\mathrm{ss}} ; \mathbf{Q}\right)
$$

as an ideal in $H_{\mathscr{G}}^{*}(\mathscr{C} ; \mathbf{Q})$.

Proof. Recall that if $\mu \in \mathscr{M}$ and $U_{\mu}=\mathscr{C}-\bigcup_{\nu>\mu} \mathscr{C}_{\nu}$ then the composition of the Thom-Gysin map

$$
H_{\mathscr{G}}^{*-2 d(\mu)}\left(\mathscr{C}_{\mu} ; \mathbf{Q}\right) \rightarrow H_{\mathscr{G}}^{*}\left(U_{\mu} ; \mathbf{Q}\right)
$$

with the restriction map

$$
H_{\mathscr{G}}^{*}\left(U_{\mu} ; \mathbf{Q}\right) \rightarrow H_{\mathscr{G}}^{*}\left(\mathscr{C}_{\mu} ; \mathbf{Q}\right)
$$

is given by multiplication by the Euler class $e_{\mu}$, which is not a zero-divisor in $H_{\mathscr{G}}^{*}\left(\mathscr{C}_{\mu} ; \mathbf{Q}\right)$. It then follows easily from the exactness of the Thom-Gysin 
sequence

$$
\cdots \rightarrow H_{\mathscr{G}}^{*-2 d(\mu)}\left(\mathscr{C}_{\mu} ; \mathbf{Q}\right) \rightarrow H_{\mathscr{G}}^{*}\left(U_{\mu} ; \mathbf{Q}\right) \rightarrow H_{\mathscr{G}}^{*}\left(U_{\mu}-\mathscr{C}_{\mu} ; \mathbf{Q}\right) \rightarrow \cdots
$$

that the direct sum of restriction maps

$$
H_{\mathscr{G}}^{*}\left(U_{\mu} ; \mathbf{Q}\right) \rightarrow H_{\mathscr{G}}^{*}\left(\mathscr{C}_{\mu} ; \mathbf{Q}\right) \oplus H_{\mathscr{G}}^{*}\left(U_{\mu}-\mathscr{C}_{\mu} ; \mathbf{Q}\right)
$$

is injective. In particular, since for given $i \geq 0$ there are only finitely many $\nu \in \mathscr{M}$ such that $2 d_{\nu} \leq i$, we can deduce that the direct sum of restriction maps

$$
H_{\mathscr{G}}^{*}\left(U_{\mu}-\mathscr{C}_{\mu} ; \mathbf{Q}\right) \rightarrow \bigoplus_{\nu \supseteq \mu} H_{\mathscr{G}}^{*}\left(\mathscr{C}_{\nu} ; \mathbf{Q}\right)
$$

is injective, and hence that the image of any element of the ideal generated by $\mathscr{R}_{\mu}$ under the restriction map

$$
H_{\mathscr{G}}^{*}(\mathscr{C} ; \mathbf{Q}) \rightarrow H_{\mathscr{G}}^{*}\left(U_{\mu}-\mathscr{C}_{\mu} ; \mathbf{Q}\right)
$$

is zero.

Suppose now, in order to obtain a contradiction, that $\eta \in H_{\mathscr{G}}^{*}(\mathscr{C} ; \mathbf{Q})$ belongs to the kernel of the restriction map

$$
H_{\mathscr{G}}^{*}(\mathscr{C} ; \mathbf{Q}) \rightarrow H_{\mathscr{G}}^{*}\left(\mathscr{C}^{\mathrm{ss}} ; \mathbf{Q}\right)
$$

but does not belong to the ideal in $H_{\mathscr{G}}^{*}(\mathscr{C} ; \mathbf{Q})$ generated by $\mathscr{R}$. Since for given $i \geq 0$ there are only finitely many $\mu \in \mathscr{M}$ such that $2 d_{\mu} \leq i$, we can choose $\mu \in \mathscr{M}$ such that the image of $\eta$ in $H_{\mathscr{G}}^{*}\left(U_{\mu} ; \mathbf{Q}\right)$ does not belong to the image of the ideal generated by $\mathscr{R}$ but such that the image of $\eta$ in $H_{\mathscr{G}}^{*}\left(U_{j l}-\mathscr{C}_{\mu} ; \mathbf{Q}\right)$ does belong to the image of the ideal generated by $\mathscr{R}$. Without loss of generality we can assume that the image of $\eta$ in $H_{\mathscr{G}}^{*}\left(U_{\mu}-\mathscr{C}_{\mu} ; \mathbf{Q}\right)$ is zero. Therefore by the exactness of the Thom-Gysin sequence there is an element $\zeta$ of $H_{\mathscr{G}}^{*-2 d(l)}\left(\mathscr{C}_{\mu} ; \mathbf{Q}\right)$ which is mapped to the image of $\eta$ in $H_{\mathscr{G}}^{*}\left(U_{\mu} ; \mathbf{Q}\right)$ by the Thom-Gysin map

$$
H_{\mathscr{G}}^{*-2 d(\mu)}\left(\mathscr{C}_{\mu} ; \mathbf{Q}\right) \rightarrow H_{\mathscr{G}}^{*}\left(U_{\mu} ; \mathbf{Q}\right) .
$$

This means that the image of $\eta$ under the restriction map

$$
H_{\mathscr{G}}^{*}(\mathscr{C} ; \mathbf{Q}) \rightarrow H_{\mathscr{G}}^{*}\left(\mathscr{C}_{\mu} ; \mathbf{Q}\right)
$$

is $e_{\mu} \zeta$. By hypothesis there is an element $\rho$ of the ideal generated by $\mathscr{R}_{\mu}$ whose image under the restriction map

$$
H_{\mathscr{G}}^{*}(\mathscr{C} ; \mathbf{Q}) \rightarrow H_{\mathscr{G}}^{*}\left(\mathscr{C}_{\mu} ; \mathbf{Q}\right)
$$

is also $e_{\mu} \zeta$. Since the images of $\rho$ and $\eta$ in $H_{\mathscr{G}}^{*}\left(U_{\mu}-\mathscr{C}_{\mu} ; \mathbf{Q}\right)$ are both zero and we have shown that the direct sum of restriction maps

$$
H_{\mathscr{G}}^{*}\left(U_{\mu} ; \mathbf{Q}\right) \rightarrow H_{\mathscr{G}}^{*}\left(\mathscr{C}_{\mu} ; \mathbf{Q}\right) \oplus H_{\mathscr{G}}^{*}\left(U_{\mu}-\mathscr{C}_{\mu} ; \mathbf{Q}\right)
$$


is injective, it follows that the images of $\rho$ and $\eta$ in $H_{\mathscr{G}}^{*}\left(U_{\mu} ; \mathbf{Q}\right)$ are the same. This contradicts the choice of $\mu$, and thus completes the proof of the proposition.

In the case when the rank $n$ is two the indexing set $\mathscr{M}$ of the stratification is simply the set of vectors $\mu=\left(d_{1}, d_{2}\right)$ where $d_{1}$ and $d_{2}$ are integers satisfying $d_{1}>d_{2}$ and $d_{1}+d_{2}=d$. The stratum $\mathscr{C}_{\mu}$ then has codimension $d_{1}-d_{2}+$ $g-1$ and its equivariant cohomology ring has generators $a_{1}^{1}$ and $a_{1}^{2}$, and $b_{1}^{1, k}$ and $b_{1}^{2, k}$ for $1 \leq k \leq 2 g$.

In order to be able to apply the last proposition we need the next result.

Proposition 2. The restriction to $H_{\mathscr{G}}^{*}\left(\mathscr{C}_{\mu} ; \mathbf{Q}\right)$ of the rth Chern class $c_{r}\left(\pi_{!}(\mathscr{V})\right)$ is the coefficient of $x^{r}$ in the power series expansion of

$$
\left(1+a_{1}^{1} x\right)^{d_{1}-\bar{g}}\left(1+a_{1}^{2} x\right)^{d_{2}-\bar{g}} \exp \left(\frac{\xi_{1} x}{1+a_{1}^{1} x}+\frac{\xi_{2} x}{1+a_{1}^{2} x}\right)
$$

where $\bar{g}=g-1$ and $\xi_{i}=\sum_{1 \leq k \leq g} b_{1}^{i, k} b_{1}^{i, k+g}$ for $i=1,2$.

Proof. First note that by the Riemann-Roch theorem

$$
\operatorname{ch}\left(\pi_{!}(V)\right)=\pi^{*}\{\operatorname{ch}(V)(1-\bar{g} \omega)\}
$$

where $\omega$ is the natural generator of $H^{2}(M, \mathbf{Z})$. The restriction to $H_{\mathscr{G}}^{*}\left(\mathscr{C}_{\mu} ; \mathbf{Q}\right)$ of the Chern character $\operatorname{ch}(V)$ is

$$
\sum_{1 \leq i \leq 2} e^{a_{1}^{i}+\sum_{k=1}^{2 g} b_{1}^{i, k} \otimes \alpha_{k}+d_{i} \omega}
$$

Therefore the restriction to $H_{\mathscr{G}}^{*}\left(\mathscr{C}_{\mu} ; \mathbf{Q}\right)$ of $\operatorname{ch}\left(\pi_{!}(V)\right)$ is

$$
e^{a_{1}^{1}}\left(\xi_{1}+d_{1}-\bar{g}\right)+e^{a_{1}^{2}}\left(\xi_{2}+d_{2}-\bar{g}\right)
$$

To obtain the Chern classes $c_{r}(E)$ of a bundle $E$ of rank : from its Chern character $\operatorname{ch}(E)$ we express the Chern character formalı; as

$$
\operatorname{ch}(E)=\sum_{k=1}^{m} e^{\gamma_{k}}
$$

where each $\gamma_{k}$ has degree two, and then $c_{r}(E)$ is the $r$ th elementary symmetric polynomial in $\gamma_{1}, \ldots, \gamma_{m}$. Equivalently we can write

$$
\sum_{r=1}^{m} c_{r}(E) x^{r}=\prod_{r=1}^{m}\left(1+\gamma_{r} x^{r}\right) \text {. }
$$

The Chern character is additive with respect to direct sums, whereas $\sum_{r=1}^{m} c_{r}(E) x^{r}$ satisfies the multiplicative property

$$
\sum_{r=1}^{m} c_{r}(E \oplus F) x^{r}=\left(\sum_{r=1}^{m} c_{r}(E) x^{r}\right)\left(\sum_{r=1}^{m} c_{r}(F) x^{r}\right) .
$$


Recall from the proof of Lemma 2 in $\S 1$ that since $\left(\xi_{1}\right)^{g+1}=0=\left(\xi_{2}\right)^{g+1}$, we can write

$$
g+\xi_{j}=\sum_{k=1}^{g} e^{\lambda_{k} \xi_{j}}
$$

for $j=1,2$, where the $r$ th elementary symmetric polynomial $\sigma_{r}$ in $\lambda_{1}, \ldots, \lambda_{g}$ is $\sigma_{r}=1 / r$ !. Since the restriction of the universal bundle $\mathscr{V}$ on $\mathscr{C} \times M$ to $\mathscr{C}_{\mu}$ (where $\mathscr{C}_{\mu}$ is identified with $\mathscr{C}_{\mu} \times\{m\}$ for some $m \in M$ ) has Chern character $e^{a_{1}^{1}}+e^{a_{1}^{2}}$, the direct sum of $k$ copies of this restriction with the restriction to $\mathscr{C}_{\mu}$ of $\pi_{!}(\mathscr{V})$ has Chern character

$$
e^{a_{1}^{1}}\left(k+d_{1}-\bar{g}+\xi_{1}\right)+e^{a_{1}^{2}}\left(k+d_{2}-\bar{g}+\xi_{2}\right)
$$

If we choose $k$ large enough that

$$
k+d_{1}-\bar{g} \geq k+d_{2}-\bar{g} \geq g
$$

then this is equal to

$$
e^{a_{1}^{1}}\left(g+\xi_{1}\right)+\left(k+d_{1}-2 g+1\right) e^{a_{1}^{1}}+\left(g+\xi_{2}\right) e^{a_{1}^{2}}+\left(k+d_{2}-2 g+1\right) e^{a_{1}^{2}},
$$

so the corresponding Chern classes are the coefficients of

$$
\left(1+a_{1}^{1} x\right)^{k+d_{1}-2 g+1}\left(1+a_{1}^{2} x\right)^{k+d_{2}-2 g+1} \prod_{i=1}^{g}\left(1+a_{1}^{1} x+\lambda_{i} \xi_{1} x\right) \prod_{j=1}^{g}\left(1+a_{1}^{2} x+\lambda_{j} \xi_{2} x\right),
$$

which equals

$$
\left(1+a_{1}^{1} x\right)^{k+d_{1}-\bar{g}}\left(1+a_{1}^{2} x\right)^{k+d_{2}-\bar{g}} \exp \left(\frac{\xi_{1} x}{1+a_{1}^{1} x}+\frac{\xi_{2} x}{1+a_{1}^{2} x}\right) .
$$

By the multiplicative property of $\sum_{r=1}^{m} c_{r}(E) x^{r}$ it follows that

$$
\sum_{r \geq 1} c_{r}\left(\pi_{!} \mathscr{V}\right) x^{r}=\left(1+a_{1}^{1} x\right)^{d_{1}-\bar{g}}\left(1+a_{1}^{2} x\right)^{d_{2}-\bar{g}} \exp \left(\frac{\xi_{1} x}{1+a_{1}^{1} x}+\frac{\xi_{2} x}{1+a_{1}^{2} x}\right)
$$

as required.

Consider the formal power series

$$
\Phi(x)=\sum_{r \geq 0} c_{r}\left(\pi_{!}(\mathscr{V})\right) x^{r}
$$

with coefficients in $H_{\mathscr{G}}^{*}(\mathscr{C} ; \mathbf{Q})$. The statement that the image of $c_{r}\left(\pi_{!}(\mathscr{V})\right)$ in the cohomology ring $H^{*}(\mathscr{M}(2, d)$; $\mathbf{Q})$ is zero if $r \geq 2 g$ is of course equivalent to the statement that the restriction of the coefficients of $\Phi(x)$ to $H^{*}(\mathscr{M}(2, d) ; \mathbf{Q})$ gives a polynomial of degree at most $2 g-1$ in $x$, and hence 
also to the statement that the restriction to $H^{*}(\mathscr{M}(2, d) ; \mathbf{Q})$ of

$$
\Psi(x)=x^{2 g-1} \Phi\left(x^{-1}\right)
$$

is a polynomial of degree at most $2 g-1$ in $x$. As $a_{1} / 2$ is an element of $H_{\mathscr{G}}^{*}(\mathscr{C} ; \mathbf{Q})$ this is also equivalent to the statement that the restriction to $H^{*}(\mathscr{M}(2, d) ; \mathbf{Q})$ of

$$
\Psi\left(x-a_{1} / 2\right) \in H_{\mathscr{G}}^{*}(\mathscr{C} ; \mathbf{Q})[[x]]
$$

is a polynomial of degree at most $2 g-1$ in $x$.

Recall that $d_{1}+d_{2}=d=4 g-3$ so that we may define a nonnegative integer $D$ and an element $\beta$ of $H_{\mathscr{G}}^{*}\left(\mathscr{C}_{\mu} ; \mathbf{Q}\right)$ by

$$
\beta=\left(a_{1}^{1}-a_{1}^{2}\right) / 2
$$

and

$$
2 D+1=d_{1}-d_{2}
$$

Then

$$
d_{1}=D+2 g-1
$$

and

$$
d_{2}=-D+2 g-2 \text {. }
$$

Corollary 1. The restriction of $\Psi\left(x-a_{1} / 2\right)$ to $H_{\mathscr{G}}^{*}\left(\mathscr{C}_{\mu} ; \mathbf{Q}\right)$ is

$$
(x+\beta)^{g+D}(x-\beta)^{\bar{g}-D} \exp \left(\frac{\xi_{1}}{x+\beta}+\frac{\xi_{2}}{x-\beta}\right) .
$$

Proof. This follows immediately from the last proposition and the fact that the restriction to $H_{\mathscr{G}}^{*}\left(\mathscr{C}_{\mu} ; \mathbf{Q}\right)$ of $a_{1}$ is $a_{1}^{1}+a_{1}^{2}$.

Next note that $\left(\xi_{1}\right)^{g+1}=0=\left(\xi_{2}\right)^{g+1}$ and we can express

$$
\begin{aligned}
(x+\beta)^{g+D}(x-\beta)^{\bar{g}-D} \exp \left(\frac{\xi_{1}}{x+\beta}+\frac{\xi_{2}}{x-\beta}\right) \\
=\sum_{k=0}^{2 g}\left(x^{2}-\beta^{2}\right)^{\bar{g}-D-k}(x+\beta)^{2 D+1} \frac{\left(\xi_{1}(x-\beta)+\xi_{2}(x+\beta)\right)^{k}}{k !}
\end{aligned}
$$

uniquely in the form

$$
\sum_{r=-\infty}^{g-1}\left(\sigma_{r}^{\mu}+\tau_{r}^{\mu} x\right)\left(x^{2}-\beta^{2}\right)^{r}
$$

for some $\sigma_{r}^{\mu}$ and $\tau_{r}^{\mu}$ in $H_{\mathscr{G}}^{*}\left(\mathscr{C}_{\mu} ; \mathbf{Q}\right)$. Moreover, as $\beta^{2}$ is the restriction to $H_{\mathscr{G}}^{*}\left(\mathscr{C}_{\mu} ; \mathbf{Q}\right)$ of $\left(\left(a_{1}\right)^{2}-4 a_{2}\right) / 2$ we can equate the formal Laurent series

$$
\Psi\left(x-a_{1} / 2\right)
$$


to a unique series of the form

$$
\sum_{r=-\infty}^{g-1}\left(\sigma_{r}+\tau_{r} x\right)\left(x^{2}+a_{2}-\left(a_{1}\right)^{2} / 4\right)^{r}
$$

for some $\sigma_{r}$ and $\tau_{r}$ in $H_{\mathscr{G}}^{*}(\mathscr{C} ; \mathbf{Q})$ whose restrictions to $H_{\mathscr{G}}^{*}\left(\mathscr{C}_{\mu} ; \mathbf{Q}\right)$ are $\sigma_{r}^{\mu}$ and $\tau_{r}^{\mu}$, respectively. We can go further and write $\sigma_{r}$ and $\tau_{r}$ uniquely in the form

$$
\sigma_{r}=\sum_{S \subseteq\{1, \ldots, 2 g\}} \sigma_{r, S} \prod_{j \in S} b_{1}^{j}
$$

and

$$
\tau_{r}=\sum_{S \subseteq\{1, \ldots, 2 g\}} \tau_{r, S} \prod_{j \in S} b_{1}^{j}
$$

where $\sigma_{r, S}$ and $\tau_{r, S}$ belong to the subring of $H_{\mathscr{G}}^{*}(\mathscr{C} ; \mathbf{Q})$ generated by $a_{1}, a_{2}$, $2 b_{2}^{j}-a_{1} b_{1}^{j}$, and $2 f_{2}-\sum_{j=1}^{g} b_{1}^{j} b_{1}^{j+g}$. The relations given by the statement that the restriction to $\mathscr{M}(2, d)$ of $\Psi\left(x-a_{1} / 2\right)$ is a polynomial of degree at most $2 g-1$ in $x$ with coefficients in $H^{*}(\mathscr{M}(2, d) ; \mathbf{Q})$ are equivalently given by the statement that the images in $H^{*}(\mathscr{M}(2, d) ; \mathbf{Q})$ of $\sigma_{r, s}$ and $\tau_{r, s}$ are zero for each $S \subseteq\{1, \ldots, 2 g\}$ if $r<0$.

We have already observed at the beginning of this section that the images $\sigma_{r, S}^{\mu}$ and $\tau_{r, S}^{\mu}$ of $\sigma_{r, S}$ and $\tau_{r, S}$ in $H_{\mathscr{G}}^{*}\left(\mathscr{C}_{\mu} ; \mathbf{Q}\right)$ are the coefficients of $\prod_{j \in S}\left(b_{1}^{1, j}+b_{1}^{2, j}\right)$ in $\sigma_{r}^{\mu}$ and $\tau_{r}^{\mu}$ expressed in terms of $a_{1}^{1}, a_{1}^{2}, b_{1}^{1, j}+b_{1}^{2, j}$, and $b_{1}^{1, j}-b_{1}^{2, j}$.

If $g \leq k \leq 2 g$ then, since $\left(\xi_{1}\right)^{g+1}=0=\left(\xi_{2}\right)^{g+1}$, there is a factor of

$$
\left(x^{2}-\beta^{2}\right)^{k-g}
$$

in $\left(\xi_{1}(x-\beta)+\xi_{2}(x+\beta)\right)^{k}$. Hence the lowest power of $\left(x^{2}-\beta^{2}\right)$ occurring in

$$
\left(x^{2}-\beta^{2}\right)^{\bar{g}-D-k}(x+\beta)^{2 D+1}\left(\xi_{1}(x-\beta)+\xi_{2}(x+\beta)\right)^{k}
$$

is at least

$$
\bar{g}-D-k+k-g=-D-1 \text {. }
$$

In other words

$$
\sigma_{r}^{\mu}=0=\tau_{r}^{\mu} \quad \text { if } r<-D-1 .
$$

In order to calculate $\sigma_{-D-1}^{\mu}$ and $\tau_{-D-1}^{\mu}$ we set

$$
(x+\beta)^{2 D+1+g-k+g}=\left(x^{2}-\beta^{2}\right) q(x)+\eta x+\zeta
$$

for some $q(x) \in H_{\mathscr{G}}^{*}\left(\mathscr{C}_{\mu} ; \mathbf{Q}\right)[x]$ and $\eta$ and $\zeta$ in $H_{\mathscr{G}}^{*}\left(\mathscr{C}_{\mu} ; \mathbf{Q}\right)$. A simple calculation shows that $\zeta=\eta \beta$ and

$$
(x+\beta)^{2 D+g-k+g}=(x-\beta) q(x)+\eta,
$$


so $\eta=(2 \beta)^{2 D+2 g-k}$. This means that if $g \leq k \leq 2 g$ then

$$
\left(x^{2}-\beta^{2}\right)^{\bar{g}-D-k}(x+\beta)^{2 D+1}\left(\xi_{1}(x-\beta)+\xi_{2}(x+\beta)\right)^{k}
$$

can be expressed as

$$
\begin{aligned}
& \left(x^{2}-\beta^{2}\right)^{-D-1}\left(\xi_{1}\right)^{k-g}\left(\xi_{2}\right)^{g}\left(\begin{array}{l}
k \\
g
\end{array}\right)(2 \beta)^{2 g-k+2 D}(x+\beta) \\
& + \text { higher powers of }\left(x^{2},-\beta^{2}\right) .
\end{aligned}
$$

Therefore

$$
\tau_{-D-1}^{\mu}=\sum_{k=g}^{2 g} \frac{\left(\xi_{1}\right)^{k-g}\left(\xi_{2}\right)^{g}}{(k-g) ! g !}(2 \beta)^{2 g-k+2 D}
$$

and

$$
\sigma_{-D-1}^{\mu}=\beta \tau_{-D-1}^{\mu}
$$

Recall that

$$
\xi_{i}=\sum_{1 \leq j \leq g} b_{1}^{i, j} b_{1}^{i, j+g}
$$

for $i=1,2$; in particular

$$
\left(\xi_{2}\right)^{g}=(-1)^{g(g-1) / 2} g ! \prod_{j=1}^{2 g} b_{1}^{2, j}
$$

Therefore as $\left(b_{1}^{2, j}\right)^{2}=0$ for all $j$ we have

$$
\left(\xi_{1}\right)^{k-g}\left(\xi_{2}\right)^{g}=\xi^{k-g}\left(\xi_{2}\right)^{g}
$$

where

$$
\xi=\sum_{1 \leq j \leq g}\left(b_{1}^{2, j}-b_{1}^{1, j}\right)\left(b_{1}^{2, j+g}-b_{1}^{1, j+g}\right) .
$$

Note that the restriction of $b_{1}^{j} \in H_{\mathscr{G}}^{*}(\mathscr{C} ; \mathbf{Q})$ to $H_{\mathscr{G}}^{*}\left(\mathscr{C}_{\mu} ; \mathbf{Q}\right)$ is $b_{1}^{1, j}+b_{1}^{2, j}$ and that $H_{\mathscr{G}}^{*}\left(\mathscr{C}_{\mu} ; \mathbf{Q}\right)$ is freely generated as a graded commutative algebra over the rationals by $a_{1}^{1}+a_{1}^{2}, a_{1}^{1}-a_{1}^{2}, b_{1}^{1, j}+b_{1}^{2, j}$, and $b_{1}^{1, j}-b_{1}^{2, j}$. We can write

$$
\left(\xi_{2}\right)^{g}=(-1)^{g(g-1) / 2} g ! 2^{-2 g} \prod_{j=1}^{2 g}\left(\left(b_{1}^{1, j}+b_{1}^{2, j}\right)+\left(b_{1}^{2, j}-b_{1}^{1, j}\right)\right)
$$

so substituting $2 \beta=a_{1}^{1}-a_{1}^{2}$ we get

$$
\begin{array}{r}
\tau_{-D-1}^{\mu}=(-1)^{g(g-1) / 2} 2^{-2 g} \sum_{k=g}^{2 g} \prod_{j=1}^{2 g}\left(\left(b_{1}^{1, j}+b_{1}^{2, j}\right)+\left(b_{1}^{2, j}-b_{1}^{1, j}\right)\right) \\
\times \frac{\xi^{k-g}}{(k-g) !}\left(a_{1}^{1}-a_{1}^{2}\right)^{2 g-k+2 D}
\end{array}
$$


The coefficient of $\prod_{j=1}^{2 g}\left(b_{1}^{1, j}+b_{1}^{2, j}\right)$ in this expression is

$$
(-1)^{g(g-1) / 2} 2^{-2 g}\left(a_{1}^{1}-a_{1}^{2}\right)^{2 D}\left(\left(a_{1}^{1}-a_{1}^{2}\right)^{g}+\xi\left(a_{1}^{1}-a_{1}^{2}\right)^{g-1}+\cdots+\xi^{g} / g !\right),
$$

and by Lemma 2 this is precisely

$$
(-1)^{g(g+1) / 2} 2^{-2 g} e_{\mu}
$$

Similarly for any subset $S$ of $\{1, \ldots, 2 g\}$ the coefficients of

$$
\prod_{j \in S}\left(b_{1}^{1, j}+b_{1}^{2, j}\right)
$$

in the expression given above for $\tau_{-D-1}^{\mu}$ and the corresponding expression for $\sigma_{-D-1}^{\mu}=\beta \tau_{-D-1}^{\mu}$ are, up to constants independent of $\mu$,

$$
e_{\mu} \prod_{j \notin S}\left(b_{1}^{1, j}-b_{1}^{2, j}\right) \text { and } e_{\mu}\left(a_{1}^{2}-a_{1}^{1}\right) \prod_{j \notin S}\left(b_{1}^{1, j}-b_{1}^{2, j}\right) .
$$

Since $H_{\mathscr{G}}^{*}\left(\mathscr{C}_{\mu} ; \mathbf{Q}\right)$ is generated by the elements $a_{1}^{2}-a_{1}^{1}$ and $b_{1}^{1, j}-b_{1}^{2, j}$ for $1 \leq j \leq 2 g$ together with the image of the restriction map

$$
H_{\mathscr{G}}^{*}(\mathscr{C} ; \mathbf{Q}) \rightarrow H_{\mathscr{G}}^{*}\left(\mathscr{C}_{\mu} ; \mathbf{Q}\right)
$$

which contains $\left(a_{1}^{2}-a_{1}^{1}\right)^{2}$, it follows that the conditions of Proposition 2 are satisfied, and hence the proof of Mumford's conjecture is complete.

Remark 3. This method for proving Mumford's conjecture when the rank $n$ is two could, in principle, be used to decide whether the conjecture is true for rank $n>2$, but the problem appears significantly more complicated in the general case.

\section{WHICH EXPRESSIONS IN THE GENERATORS ARE RELATIONS?}

This section contains a method for determining when a polynomial expression in the rational generators $a_{r}, b_{r}^{j}$, and $f_{r}$ for the cohomology ring of the moduli space $\mathscr{M}(n, d)$ is a relation ${ }^{4}$ when the rank $n$ is two. We shall start by discussing the general case when $n \geq 2$.

Consider the subset $\Delta$ of the indexing set $\mathscr{M}$ consisting of those elements $\mu=\left(\mu_{1}, \ldots, \mu_{n}\right)$ in $\mathscr{M}$ satisfying $\mu_{1}>\mu_{2}>\cdots>\mu_{n}$. Equivalently $\Delta$ is the set of all $\delta=\left(\delta_{1}, \ldots, \delta_{n}\right) \in \mathbf{Z}^{n}$ satisfying $\delta_{1}+\cdots+\delta_{n}=d$ and

\footnotetext{
${ }^{4}$ Of course Mumford's conjecture tells us that such an expression is a relation if and only if it belongs to the ideal generated by the set of relations $\mathscr{R}$ described in $\S 2$, but in practice it is difficult to decide whether or not a given expression belongs to this ideal.
} 
$\delta_{1}>\delta_{2}>\cdots>\delta_{n}$. We shall show firstly that the direct sum of restriction maps

$$
H_{\mathscr{G}}^{*}(\mathscr{C} ; \mathbf{Q}) \rightarrow \bigoplus_{\delta \in \Delta} H_{\mathscr{G}}^{*}\left(\mathscr{C}_{\delta} ; \mathbf{Q}\right)
$$

is injective, and secondly that at least when the rank $n$ is two there is a description, in terms of the rational generators $a_{1}^{j}, b_{1}^{j, k}$ for the cohomology rings $H_{\mathscr{G}}^{*}\left(\mathscr{C}_{\delta} ; \mathbf{Q}\right)$, of the image under this injection of the kernel of the restriction map $H_{\mathscr{G}}^{*}(\mathscr{C} ; \mathbf{Q}) \rightarrow H_{\mathscr{G}}^{*}\left(\mathscr{C}^{\text {ss }} ; \mathbf{Q}\right)$. Since the images under the restriction maps

$$
H_{\mathscr{G}}^{*}(\mathscr{C} ; \mathbf{Q}) \rightarrow H_{\mathscr{G}}^{*}\left(\mathscr{C}_{\delta} ; \mathbf{Q}\right)
$$

of the rational generators $a_{r}, b_{r}^{j}$, and $f_{r}$ for the cohomology ring $H_{\mathscr{G}}^{*}(\mathscr{C} ; \mathbf{Q})$ can be written down explicitly in terms of the generators $a_{1}^{j}, b_{1}^{j, k}$ (see the proof of Proposition 3), this gives us a way to determine when $n=2$ which polynomials in the generators lie in the kernel of the restriction map $H_{\mathscr{G}}^{*}(\mathscr{C} ; \mathbf{Q}) \rightarrow$ $H_{\mathscr{G}}^{*}\left(\mathscr{C}^{\text {ss }} ; \mathbf{Q}\right)$ and hence which polynomials are relations in $H^{*}(\mathscr{M}(n, d) ; \mathbf{Q})$.

Proposition 3. The direct sum of restriction maps

$$
H_{\mathscr{G}}^{*}(\mathscr{C} ; \mathbf{Q}) \rightarrow \bigoplus_{\delta \in \Delta} H_{\mathscr{G}}^{*}\left(\mathscr{C}_{\delta} ; \mathbf{Q}\right)
$$

is injective.

Proof. Since the only relations between the generators $a_{r}, b_{r}^{j}$, and $f_{r}$ for $H_{\mathscr{G}}^{*}(\mathscr{C} ; \mathbf{Q})$ are that the $a_{r}$ and $f_{r}$ commute with everything and the $b_{r}^{j}$ anticommute with each other, a general element $\tau$ of $H_{\mathscr{G}}^{*}(\mathscr{C} ; \mathbf{Q})$ can be expressed uniquely in the form

$$
\tau=\sum_{p \in \mathscr{P}, q \in \mathscr{Q}, s \in \mathscr{S}} m(p, q, s) \prod_{r=1}^{n}\left(a_{r}\right)^{p_{r}} \prod_{r=1}^{n} \prod_{j=1}^{2 g}\left(b_{r}^{j}\right)^{q_{r, j}} \prod_{r=2}^{n}\left(f_{r}\right)^{s_{r}}
$$

where $\mathscr{P}, \mathscr{Q}$, and $\mathscr{S}$ are the sets of sequences $p=\left(p_{1}, \ldots, p_{n}\right), q=$ $\left(q_{1,1}, \ldots, q_{n, 2 g}\right)$, and $s=\left(s_{2}, \ldots, s_{n}\right)$ of nonnegative integers such that $q_{r, j}=0$ or 1 for each $r$ and $j$, and where $m(p, q, s) \in \mathbf{Q}$ for each $p \in$ $\mathscr{P}, q \in \mathscr{Q}, s \in \mathscr{S}$. Let us assume that the restriction of $\tau$ to $H_{\mathscr{G}}^{*}\left(\mathscr{C}_{\delta} ; \mathbf{Q}\right)$ is zero for every $\delta \in \Delta$. We need to show that $\tau=0$.

In terms of the generators $a_{1}^{1}, \ldots, a_{1}^{n}$ and $b_{1}^{1,1}, \ldots, b_{1}^{n, 2 g}$ for $H_{\mathscr{G}}^{*}\left(\mathscr{C}_{\delta} ; \mathbf{Q}\right)$ the restriction map

$$
H_{\mathscr{G}}^{*}(\mathscr{C} ; \mathbf{Q}) \rightarrow H_{\mathscr{G}}^{*}\left(\mathscr{C}_{\delta} ; \mathbf{Q}\right)
$$

is determined by equating the coefficients of powers of $x$ in two polynomials, the first of which is

$$
\prod_{k=1}^{n}\left(1+\left(a_{1}^{k} \otimes 1+\sum_{j=1}^{2 g} b_{1}^{k, j} \otimes \alpha_{j}+\delta_{k} \omega\right) x\right)
$$


and the second of which is the restriction to $H_{\mathscr{G}}^{*}\left(\mathscr{C}_{\delta} \times M ; \mathbf{Q}\right)$ of

$$
1+\sum_{r=1}^{n}\left(a_{r} \otimes 1+\sum_{j=1}^{2 g} b_{r}^{j} \otimes \alpha_{j}+f_{r} \omega\right) x^{r}
$$

This means that if $\sigma_{r}^{n}\left(x_{j}\right)$ denotes the $r$ th elementary symmetric polynomial in $n$ variables $x_{1}, \ldots, x_{n}$ then the restriction of $\tau$ to $H_{\mathscr{G}}^{*}\left(\mathscr{C}_{\delta} ; \mathbf{Q}\right)$ is

$$
\begin{gathered}
\sum_{p \in \mathscr{P}, q \in \mathscr{Q}, s \in \mathscr{S}} m(p, q, s) \prod_{r=1}^{n} \sigma_{r}^{n}\left(a_{1}^{j}\right)^{p_{r}} \prod_{r=1}^{n} \prod_{j=1}^{2 g}\left(\sum_{k=1}^{n} b_{1}^{k, j} \sigma_{r-1}^{n-1}\left(a_{1}^{i} ; i \neq k\right)\right)^{q_{r, j}} \\
\times \prod_{r=2}^{n}\left(\sum_{k=1}^{n} \delta_{k} \sigma_{r-1}^{n-1}\left(a_{1}^{i} ; i \neq k\right)+\sum_{k \neq \ell} \sum_{j=1}^{2 g} b_{1}^{k, j} b_{1}^{\ell, g+j} \sigma_{r-2}^{n-2}\left(a_{1}^{i} ; i \neq k, \ell\right)\right)^{s_{r}} .
\end{gathered}
$$

We are assuming that this is zero for every $\delta \in \Delta$. Since the only relations between the generators $a_{1}^{1}, \ldots, a_{1}^{n}$ and $b_{1}^{1,1}, \ldots, b_{1}^{n, 2 g}$ for $H_{\mathscr{G}}^{*}\left(\mathscr{C}_{\delta} ; \mathbf{Q}\right)$ are that the $a_{1}^{k}$ commute with everything and the $b_{1}^{k, j}$ anticommute with each other, the expression above can be written uniquely as a linear combination of monomials of the form

$$
\left(a_{1}^{1}\right)^{u_{1}} \cdots\left(a_{1}^{n}\right)^{u_{n}}\left(b_{1}^{1,1}\right)^{v_{1,1}} \cdots\left(b_{1}^{n, 2 g}\right)^{v_{n, 2 g}}
$$

whose coefficients are rational polynomials in $\delta_{1}, \ldots, \delta_{n}$. Since the restriction of $\tau$ to $H_{\mathscr{G}}^{*}\left(\mathscr{C}_{\delta} ; \mathbf{Q}\right)$ is zero for every $\delta \in \Delta$, these polynomials vanish whenever $\left(\delta_{1}, \ldots, \delta_{n}\right) \in \Delta$. At this point we need a lemma whose proof will be deferred unti' later.

Lemma 3. Let $\phi\left(x_{1}, \ldots, x_{n}\right) \in \mathbf{C}\left[x_{1}, \ldots, x_{n}\right]$ be a polynomial such that

$$
\phi\left(\delta_{1}, \ldots, \delta_{n}\right)=0
$$

whenever $\left(\delta_{1}, \ldots, \delta_{n}\right) \in \mathbf{Z}^{n}$ satisfies

$$
\delta_{1}+\cdots+\delta_{n}=d \text { and } \delta_{1}>\delta_{2}>\cdots>\delta_{n} .
$$

Then $\phi\left(\delta_{1}, \ldots, \delta_{n}\right)=0$ whenever $\delta_{1}, \ldots, \delta_{n}$ are complex numbers satisfying $\delta_{1}+\cdots+\delta_{n}=d$.

Given this lemma it follows that we can formally substitute $\delta_{n}=0, a_{1}^{n}=0$, and $b_{1}^{n, j}=0$ for $1 \leq j \leq 2 g$ in the expression for the restriction of $\tau$ above and obtain zero whenever $\delta_{1}+\cdots+\delta_{n-1}=d$. This substitution gives us the same result as substituting $a_{n}=0, f_{n}=0$, and $b_{n}^{j}=0$ for $1 \leq j \leq 2 g$ in $\tau$ and replacing $n$ by $n-1$. By induction on $n$ it follows that this substitution in $\tau$ gives zero. Therefore since the only relations between the generators $a_{r}$, $b_{r}^{j}$, and $f_{r}$ for $H_{\mathscr{G}}^{*}(\mathscr{C} ; \mathbf{Q})$ are that the $a_{r}$ and $f_{r}$ commute with everything 
and the $b_{r}^{j}$ anticommute with each other, $\tau$ must be of the form

$$
\tau=a_{n} w+\sum_{j=1}^{2 g} b_{n}^{j} y_{j}+f_{n} z
$$

for some $w, y_{j}, z \in H_{\mathscr{G}}^{*}(\mathscr{C} ; \mathbf{Q})$. Now making the substitutions $a_{1}^{n}=0$ and $b_{1}^{n, j}=0$ for $1 \leq j \leq 2 g$ without the substitution $\delta_{n}=0$, we find that the result of these substitutions in the restriction of $z$ to $H_{\mathscr{G}}^{*}\left(\mathscr{C}_{\delta} ; \mathbf{Q}\right)$ is zero. Arguing as above we find that $z$ must be of the form

$$
z=a_{n} \hat{w}+\sum_{j=1}^{2 g} b_{n}^{j} \hat{y}_{j}+f_{n} \hat{z}
$$

for some $\hat{w}, \hat{y}_{j}, \hat{z} \in H_{\mathscr{G}}^{*}(\mathscr{C} ; \mathbf{Q})$. It follows that by modifying $w$ and the $y_{j}$ we can assume

$$
\tau=a_{n} w+\sum_{j=1}^{2 g} b_{n}^{j} y_{j}+\left(f_{n}\right)^{2} \hat{z},
$$

and continuing to repeat the argument we find that by modifying $w$ and the $y_{j}$ we can assume

$$
\tau=a_{n} w+\sum_{j=1}^{2 g} b_{n}^{j} y_{j}
$$

for some $w, y_{j} \in H_{\mathscr{G}}^{*}(\mathscr{C} ; \mathbf{Q})$. Next we make the substitutions $a_{1}^{n}=0$ and $b_{1}^{n, j}=0$ for $1 \leq j \leq 2 g-1$ without the substitutions $\delta_{n}=0$ and $b_{1}^{n, 2 g}=0$ and find that the result of making these substitutions in $y_{2 g}$ is zero, so that $b_{j}$ the argument above

$$
y_{2 g}=a_{n} \tilde{w}+\sum_{j=1}^{2 g} b_{n}^{j} \tilde{y}_{j}
$$

for some $\tilde{w}, \tilde{y}_{j} \in H_{\mathscr{G}}^{*}(\mathscr{C} ; \mathbf{Q})$. Thus by modifying $w$ ar $^{r}$ the $y_{j}$ for $1 \leq j<$ $2 \mathrm{~g}$ we can assume

$$
\tau=a_{n} w+\sum_{j=1}^{2 g-1} b_{n}^{j} y_{j}+\left(b_{n}^{2 g}\right)^{2} y_{2 g}
$$

and repetition of the argument enough times tells us that we can assume

$$
\tau=a_{n} w+\sum_{j=1}^{2 g-1} b_{n}^{j} y_{j} .
$$

Continuing this process we eventually find that $\tau$ is of the form $\tau=a_{n} w$ for some $w \in H_{\mathscr{G}}^{*}(\mathscr{C} ; \mathbf{Q})$. It then follows that the restriction of $w$ to $H_{\mathscr{G}}^{*}\left(\mathscr{C}_{\delta} ; \mathbf{Q}\right)$ is zero for every $\delta \in \Delta$, and hence by induction on the degree of $\tau$ that $w=0$. Therefore $\tau=0$ as required.

It remains to prove Lemma 3. 
Proof of Lemma 3. Define a polynomial $q$ in $n-1$ variables by

$$
q\left(X_{1}, \ldots, X_{n-1}\right)=p\left(X_{1}, \ldots, X_{n-1}, d-X_{1}-\cdots-X_{n-1}\right) .
$$

What we need to prove is that if $q\left(\delta_{1}, \ldots, \delta_{n-1}\right)$ vanishes whenever $\delta_{1}, \ldots$, $\delta_{n-1}$ are integers satisfying

$$
\delta_{1}>\delta_{2}>\cdots>\delta_{n-1}>d-\delta_{1}-\cdots-\delta_{n-1},
$$

then $q$ vanishes identically. The proof is by induction on $n$; when $n=1$ there is nothing to prove.

Suppose that $n \geq 2$. Choose integers $\delta_{2}, \ldots, \delta_{n-1}$ satisfying

$$
\delta_{2}>\delta_{3}>\cdots>\delta_{n-1}
$$

and consider the polynomial $q\left(X, \delta_{2}, \ldots, \delta_{n-1}\right)$ in $X$. If $n=2$ this vanishes at $X=\delta_{1}$ whenever $\delta_{1}$ is an integer satisfying $\delta_{1}>d-\delta_{1}$, and if $n>2$ it vanishes at $X=\delta_{1}$ whenever $\delta_{1}$ is an integer satisfying $\delta_{1}>\delta_{2}$ and $\delta_{n-1}>d-\delta_{1}-\delta_{2}-\cdots-\delta_{n-1}$, that is, $\delta_{1}>d-\delta_{2}-\cdots-\delta_{n-2}-2 \delta_{n-1}$. In each case there are infinitely many such integers $\delta_{1}$, so the polynomial $q\left(X, \delta_{2}, \ldots, \delta_{n-1}\right)$ must vanish identically. Therefore given any $\delta_{1} \in \mathbf{C}$, the polynomial

$$
q_{\delta_{1}}\left(X_{1}, \ldots, X_{n-2}\right)=q\left(\delta_{1}, X_{1}, \ldots, X_{n-2}\right)
$$

vanishes at $\left(\delta_{2}, \ldots, \delta_{n}\right)$ whenever $\delta_{2}, \ldots, \delta_{n}$ are integers satisfying $\delta_{2}>$ $\delta_{3}>\cdots>\delta_{n-1}$. It follows by induction that $q_{\delta_{1}}$ vanishes identically for any $\delta_{1} \in \mathbf{C}$. This completes the proof of Lemma 3 and hence also of Proposition 3.

Next note that it follows from Proposition 2 that the relation

$$
(g-1) a_{1}=c_{1}\left(\pi_{!}(V)\right)
$$

in $H^{*}(\mathscr{M}(n, d) ; \mathbf{Q})$ can be rewritten in terms of the generators $a_{r}, b_{r}^{j}$, and $f_{r}$ as

$$
(g-1) a_{1}=(d-g+1) a_{1}-f_{2}+\sum_{1 \leq k \leq g} b_{1}^{k} b_{1}^{k+g}
$$

or equivalently

$$
(d-2 g+2) a_{1}=f_{2}-\sum_{1 \leq k \leq g} b_{1}^{k} b_{1}^{k+g} .
$$

Recall that this means that a polynomial in the rational generators $a_{r}, b_{r}^{j}$, and $f_{r}$ for the cohomology ring $H_{\mathscr{G}}^{*}(\mathscr{C} ; \mathbf{Q})$ is a relation in $H^{*}(\mathscr{M}(n, d) ; \mathbf{Q})$ precisely when it lies in the sum of the ideal generated by

$$
(d-2 g+2) a_{1}-f_{2}+\sum_{1 \leq k \leq g} b_{1}^{k} b_{1}^{k+g}
$$


and the kernel of the restriction map $H_{\mathscr{G}}^{*}(\mathscr{C} ; \mathbf{Q}) \rightarrow H_{\mathscr{G}}^{*}\left(\mathscr{C}^{\text {ss }} ; \mathbf{Q}\right)$. It therefore follows from Proposition 3 that in order to determine whether a polynomial in the rational generators $a_{r}, b_{r}^{j}$, and $f_{r}$ for the cohomology ring $H_{\mathscr{G}}^{*}(\mathscr{C} ; \mathbf{Q})$ is a relation in $H^{*}(\mathscr{M}(n, d) ; \mathbf{Q})$, it suffices to know whether its image under the direct sum of restriction maps

$$
H_{\mathscr{G}}^{*}(\mathscr{C} ; \mathbf{Q}) \rightarrow \bigoplus_{\delta \in \Delta} H_{\mathscr{G}}^{*}\left(\mathscr{C}_{\delta} ; \mathbf{Q}\right)
$$

is contained in the image of the sum of the ideal generated by

$$
(d-2 g+2) a_{1}-f_{2}+\sum_{1 \leq k \leq g} b_{1}^{k} b_{1}^{k+g}
$$

and the kernel of the restriction map $H_{\mathscr{G}}^{*}(\mathscr{C} ; \mathbf{Q}) \rightarrow H_{\mathscr{G}}^{*}\left(\mathscr{C}^{\text {ss }} ; \mathbf{Q}\right)$. Moreover, we have already seen in the proof of Proposition 3 how to write down explicitly the image in $H_{\mathscr{G}}^{*}\left(\mathscr{C}_{\delta} ; \mathbf{Q}\right)$ of any such polynomial expression

$$
\sum_{p \in \mathscr{P}, q \in \mathscr{Q}, s \in \mathscr{S}} m(p, q, s) \prod_{r=1}^{n}\left(a_{r}\right)^{p_{r}} \prod_{r=1}^{n} \prod_{j=1}^{2 g}\left(b_{r}^{j}\right)^{q_{r, j}} \prod_{r=2}^{n}\left(f_{r}\right)^{s_{r}} .
$$

Thus it suffices to determine explicitly the image in $\bigoplus_{\delta \in \Delta} H_{\mathscr{G}}^{*}\left(\mathscr{C}_{\delta} ; \mathbf{Q}\right)$ of the kernel of the restriction map $H_{\mathscr{G}}^{*}(\mathscr{C} ; \mathbf{Q}) \rightarrow H_{\mathscr{G}}^{*}\left(\mathscr{C}^{\text {ss }} ; \mathbf{Q}\right)$.

Recall further that from Lemma 1 that the Thom-Gysin maps

$$
H_{\mathscr{G}}^{j-2 d(\mu)}\left(\mathscr{C}_{\mu} ; \mathbf{Q}\right) \rightarrow H_{\mathscr{G}}^{j}\left(U_{\mu} ; \mathbf{Q}\right)
$$

can be lifted to maps $H_{\mathscr{G}}^{j-2 d(\mu)}\left(\mathscr{C}_{\mu} ; \mathbf{Q}\right) \rightarrow H_{\mathscr{G}}^{j}(\mathscr{C} ; \mathbf{Q})$ and that the direct sum

$$
\bigoplus_{\mu \neq \mu_{0}} H_{\mathscr{G}}^{j-2 d(\mu)}\left(\mathscr{C}_{\mu} ; \mathbf{Q}\right) \rightarrow H_{\mathscr{G}}^{j}(\mathscr{C} ; \mathbf{Q})
$$

of any choice of such lifts is an isomorphism onto its image, which is the kernel of the restriction map $H_{\mathscr{G}}^{j}(\mathscr{C} ; \mathbf{Q}) \rightarrow H_{\mathscr{G}}^{j}\left(\mathscr{C}^{\text {ss }} ; \mathbf{Q}\right)$. This means that our task will be complete if we can describe explicitly the compositions

$$
H_{\mathscr{G}}^{j-2 d(\mu)}\left(\mathscr{C}_{\mu} ; \mathbf{Q}\right) \rightarrow H_{\mathscr{G}}^{j}\left(\mathscr{C}_{\delta} ; \mathbf{Q}\right)
$$

of some suitably chosen lifts $H_{\mathscr{G}}^{j-2 d(\mu)}\left(\mathscr{C}_{\mu} ; \mathbf{Q}\right) \rightarrow H_{\mathscr{G}}^{j}(\mathscr{C} ; \mathbf{Q})$ of the Thom-Gysin maps $H_{\mathscr{G}}^{j-2 d(\mu)}\left(\mathscr{C}_{\mu} ; \mathbf{Q}\right) \rightarrow H_{\mathscr{G}}^{j}\left(U_{\mu} ; \mathbf{Q}\right)$ with the restriction maps

$$
H_{\mathscr{G}}^{j}(\mathscr{C} ; \mathbf{Q}) \rightarrow H_{\mathscr{G}}^{j}\left(\mathscr{C}_{\delta} ; \mathbf{Q}\right)
$$

This is done in the case when the rank $n$ is two by the next three propositions.

Note also that when the rank $n$ is two then $\Delta=\mathscr{M}-\left\{\mu_{0}\right\}$. 
Proposition 4. If $\delta \nsupseteq \mu$ then the composition

$$
H_{\mathscr{G}}^{j-2 d(\mu)}\left(\mathscr{C}_{\mu} ; \mathbf{Q}\right) \rightarrow H_{\mathscr{G}}^{j}\left(\mathscr{C}_{\delta} ; \mathbf{Q}\right)
$$

of any choice of lift $H_{\mathscr{G}}^{j-2 d(\mu)}\left(\mathscr{C}_{\mu} ; \mathbf{Q}\right) \rightarrow H_{\mathscr{G}}^{j}(\mathscr{C} ; \mathbf{Q})$ of the Thom-Gysin map $H_{\mathscr{G}}^{j-2 d(\mu)}\left(\mathscr{C}_{\mu} ; \mathbf{Q}\right) \rightarrow H_{\mathscr{G}}^{j}\left(U_{\mu} ; \mathbf{Q}\right)$ with the restriction maps

$$
H_{\mathscr{G}}^{j}(\mathscr{C} ; \mathbf{Q}) \rightarrow H_{\mathscr{G}}^{j}\left(\mathscr{C}_{\delta} ; \mathbf{Q}\right)
$$

is zero.

Proof. Since $\mathscr{C}_{f}$ is contained in $U_{\mu}-\mathscr{C}_{\mu}$, the composition factors through the composition of two maps in a Thom-Gysin sequence. It is therefore zero because Thom-Gysin sequences are exact.

Proposition 5. If $\delta=\mu$ then the composition

$$
H_{\mathscr{G}}^{j-2 d(\mu)}\left(\mathscr{C}_{\mu} ; \mathbf{Q}\right) \rightarrow H_{\mathscr{G}}^{j}\left(\mathscr{C}_{\delta} ; \mathbf{Q}\right)
$$

of any choice of lift $H_{\mathscr{G}}^{j-2 d(\mu)}\left(\mathscr{C}_{\mu} ; \mathbf{Q}\right) \rightarrow H_{\mathscr{G}}^{j}(\mathscr{C} ; \mathbf{Q})$ of the Thom-Gysin map $H_{\mathscr{G}}^{j-2 d(\mu)}\left(\mathscr{C}_{\mu} ; \mathbf{Q}\right) \rightarrow H_{\mathscr{G}}^{j}\left(U_{\mu} ; \mathbf{Q}\right)$ with the restriction maps

$$
H_{\mathscr{G}}^{j}(\mathscr{C} ; \mathbf{Q}) \rightarrow H_{\mathscr{G}}^{j}\left(\mathscr{C}_{\delta} ; \mathbf{Q}\right)
$$

is multiplication by the equivariant Euler class $e_{\mu}$ of the normal bundle to $\mathscr{C}_{\mu}$ in $\mathscr{C}$.

Proof. The result comes from chasing through the definition of the Thom-Gysin map.

Remark 4. Recall from Lemma 2 of $\S 1$ that when the rank $n$ is two the equivariant Euler class $e_{\mu}$ is given by the expression

$$
\left(a_{1}^{2}-a_{1}^{1}\right)^{d_{1}-d_{2}-1}\left(\left(a_{1}^{2}-a_{1}^{1}\right)^{g}-\xi\left(a_{1}^{2}-a_{1}^{1}\right)^{g-1}+\frac{(\xi)^{2}}{2 !}\left(a_{1}^{2}-a_{1}^{1}\right)^{g-2}+\cdots+\frac{\left.(-\xi)^{g}\right)}{g !}\right.
$$

where

$$
\xi=\sum_{1 \leq k \leq g}\left(b_{1}^{2, k}-b_{1}^{1, k}\right)\left(b_{1}^{2, k+g}-b_{1}^{1, k+g}\right)
$$

Recall that the only relations satisfied by the generators $a_{1}^{i}$ and $b_{1}^{i, j}$ of the cohomology ring $H_{\mathscr{G}}^{*}\left(\mathscr{C}_{\mu} ; \mathbf{Q}\right)$ are that the $a_{1}^{i}$ commute with everything and the $b_{1}^{i, j}$ anticommute with each other. Note also that any symmetric polynomial in $a_{1}^{1}$ and $a_{1}^{2}$ can be expressed uniquely as a polynomial in the elementary symmetric polynomials $a_{1}^{1}+a_{1}^{2}$ and $a_{1}^{1} a_{1}^{2}$ and that any polynomial in $a_{1}^{1}$ and $a_{1}^{2}$ can be expressed uniquely as $p+\left(a_{1}^{1}-a_{1}^{2}\right) q$ where $p$ and $q$ are symmetric. 
Therefore any $c \in H_{\mathscr{G}}^{*}\left(\mathscr{C}_{\mu} ; \mathbf{Q}\right)$ can be expressed uniquely in the form

$$
\begin{aligned}
c= & \sum_{S \subseteq\{1, \ldots, 2 g\}}\left(p_{S}\left(a_{1}^{1}+a_{1}^{2}, a_{1}^{1} a_{1}^{2}, b_{1}^{1, j}+b_{1}^{2, j}\right)\right. \\
& \left.+\left(a_{1}^{1}-a_{1}^{2}\right) q_{S}\left(a_{1}^{1}+a_{1}^{2}, a_{1}^{1} a_{1}^{2}, b_{1}^{1, j}+b_{1}^{2, j}\right)\right) \prod_{j \notin S}\left(b_{1}^{1, j}-b_{1}^{2, j}\right) .
\end{aligned}
$$

Hence we can define a map

$$
\rho_{\mu, \delta}: H_{\mathscr{G}}^{*}\left(\mathscr{C}_{\mu} ; \mathbf{Q}\right) \rightarrow H_{\mathscr{G}}^{*}\left(\mathscr{C}_{\delta} ; \mathbf{Q}\right)
$$

which takes $c$ to

$$
\begin{aligned}
\sum_{S \subseteq\{1, \ldots, 2 g\}}\left(\tau_{-D-1, S}^{\delta} p_{S}\left(a_{1}^{1}+a_{1}^{2}, a_{1}^{1} a_{1}^{2}, b_{1}^{1, j}+b_{1}^{2, j}\right)\right. \\
\left.\quad+2 \sigma_{-D-1, S}^{\delta} q_{S}\left(a_{1}^{1}+a_{1}^{2}, a_{1}^{1} a_{1}^{2}, b_{1}^{1, j}+b_{1}^{2, j}\right)\right) \prod_{j \notin S}\left(b_{1}^{1, j}-b_{1}^{2, j}\right),
\end{aligned}
$$

where we use the same notation for the generators $a_{1}^{i}$ and $b_{1}^{i, j}$ of $H_{\mathscr{G}}^{*}\left(\mathscr{C}_{\mu} ; \mathbf{Q}\right)$ and $H_{\mathscr{G}}^{*}\left(\mathscr{C}_{\delta} ; \mathbf{Q}\right)$, and where $d_{1}-d_{2}=2 D+1$ and $\sigma_{r, S}^{\delta}$ and $\tau_{r, S}^{\delta}$ are defined as in the last section by expressing

$$
\begin{aligned}
(x+ & \left.\left(a_{1}^{1}-a_{1}^{2}\right) / 2\right)^{g+D}\left(x-\left(a_{1}^{1}-a_{1}^{2}\right) / 2\right)^{\bar{g}-D} \\
& \times \exp \left(\frac{\xi_{1}}{x+\left(a_{1}^{1}-a_{1}^{2}\right) / 2}+\frac{\xi_{2}}{x-\left(a_{1}^{1}-a_{1}^{2}\right) / 2}\right),
\end{aligned}
$$

uniquely in the form

$$
\sum_{r=-\infty}^{g-1} \sum_{S \subseteq\{1, \ldots, 2 g\}}\left(\sigma_{r, S}^{\mu}+\tau_{r, S}^{\mu} x\right)\left(x^{2}-\left(a_{1}^{1}-a_{1}^{2}\right)^{2} / 4\right)^{r} \prod_{j \notin S}\left(b_{1}^{1, j}-b_{1}^{2, j}\right) .
$$

Proposition 6. If the rank $n$ is two and $\mu \in \mathscr{M}-\left\{\mu_{0}\right\}=\Delta$ there exist lifts $H_{\mathscr{G}}^{j-2 d(\mu)}\left(\mathscr{C}_{\mu} ; \mathbf{Q}\right) \rightarrow H_{\mathscr{G}}^{j}(\mathscr{C} ; \mathbf{Q})$ of the Thom-Gysin maps $H_{\mathscr{G}}^{j-2 d(\mu)}\left(\mathscr{C}_{\mu} ; \mathbf{Q}\right) \rightarrow$ $H_{\mathscr{G}}^{j}\left(U_{\mu} ; \mathbf{Q}\right)$ whose compositions

$$
H_{\mathscr{G}}^{j-2 d(\mu)}\left(\mathscr{C}_{\mu} ; \mathbf{Q}\right) \rightarrow H_{\mathscr{G}}^{j}\left(\mathscr{C}_{\delta} ; \mathbf{Q}\right)
$$

with the restriction maps

$$
H_{\mathscr{G}}^{j}(\mathscr{C} ; \mathbf{Q}) \rightarrow H_{\mathscr{G}}^{j}\left(\mathscr{C}_{\delta} ; \mathbf{Q}\right)
$$

are given by the maps

$$
\rho_{\mu, \delta}: H_{\mathscr{G}}^{*}\left(\mathscr{C}_{\mu} ; \mathbf{Q}\right) \rightarrow H_{\mathscr{G}}^{*}\left(\mathscr{C}_{\delta} ; \mathbf{Q}\right)
$$

just defined if $\delta>\mu$.

Proof. Since the Thom-Gysin maps $H_{\mathscr{G}}^{j-2 d(\mu)}\left(\mathscr{C}_{\mu} ; \mathbf{Q}\right) \rightarrow H_{\mathscr{G}}^{j}\left(U_{\mu} ; \mathbf{Q}\right)$ respect the $H_{\mathscr{G}}^{*}(\mathscr{C} ; \mathbf{Q})$-module structure, it suffices to check that for any $S \subseteq\{1, \ldots, 2 g\}$ 
the images of

$$
\prod_{j \notin S}\left(b_{1}^{1, j}-b_{1}^{2, j}\right) \text { and }\left(a_{1}^{1}-a_{1}^{2}\right) \prod_{j \notin S}\left(b_{1}^{1, j}-b_{1}^{2, j}\right)
$$

under the Thom-Gysin maps are the same as the restrictions to $U_{\mu}$ of the elements $\tau_{-D-1, S}$ and $\sigma_{-D-1, S}$ of $H_{\mathscr{G}}^{*}(\mathscr{C} ; \mathbf{Q})$ defined in the last section. Since it was shown during the proof of Proposition 1 in $\S 1$ that the direct sum of restriction maps

$$
H_{\mathscr{G}}^{*}\left(U_{\mu} ; \mathbf{Q}\right) \rightarrow H_{\mathscr{G}}^{*}\left(\mathscr{C}^{\text {ss }} ; \mathbf{Q}\right) \oplus \bigoplus_{\delta \leq \mu} H_{\mathscr{G}}^{*}\left(\mathscr{C}_{\delta} ; \mathbf{Q}\right)
$$

is injective, this follows directly from the results of the last section together with the last two propositions.

\section{Proof of the Newstead-Ramanan conjecture}

The aim of this section is to prove the following theorem, which was conjectured by Newstead and Ramanan.

Theorem 2. The Pontryagin ring of the tangent bundle to the moduli space $\mathscr{M}(2, d)$ of semistable bundles of rank two and odd degree $d$ over a fixed Riemann surface $M$ of genus $g \geq 2$ vanishes in dimensions greater than $4(g-1)$.

Here the Pontryagin ring of the tangent bundle is the subring of the cohomology ring $H^{*}(\mathscr{M}(2, d) ; \mathbf{Q})$ generated by all the Pontryagin classes of the tangent bundle. As shown in $\S 4$ of [16, Corollary 2 to Theorem 2] and also $\S 9$ of $[2$, p. 584$]$ the Pontryagin ring is actually generated by the first Pontryagin class $p_{1}$. Thus to prove the theorem it suffices to show that $\left(p_{1}\right)^{g}=0$.

Recall from $\S 1$ that the rational cohomology ring of the moduli space $\mathscr{M}(2, d)$ is generated by elements

for $1 \leq j \leq 2 g$ and

$$
\begin{gathered}
a_{1} \in H^{2}(\mathscr{M}(2, d) ; \mathbf{Q}), \\
a_{2} \in H^{4}(\mathscr{M}(2, d) ; \mathbf{Q}), \\
b_{1}^{j} \in H^{1}(\mathscr{M}(2, d) ; \mathbf{Q}), \\
b_{2}^{j} \in H^{3}(\mathscr{M}(2, d) ; \mathbf{Q})
\end{gathered}
$$

$$
f_{2} \in H^{2}(\mathscr{M}(2, d) ; \mathbf{Q}) \text {. }
$$

These generators correspond to generators of the equivariant cohomology ring $H_{\mathscr{G}}^{*}(\mathscr{C} ; \mathbf{Q})$, which by an abuse of notation have been denoted by the same symbols. In terms of these generators we have

$$
p_{1}=2(g-1)\left(\left(a_{1}\right)^{2}-4 a_{2}\right)
$$

by $[16, \S 4$, Corollary 2 to Theorem 2$]$. Thus to prove the theorem it suffices to show that $\left(\left(a_{1}\right)^{2}-4 a_{2}\right)^{g}=0$ in $H^{*}(\mathscr{M}(2, d) ; \mathbf{Q})$. 
Recall also that if $\delta \in \Delta$ the restriction map

$$
H_{\mathscr{G}}^{*}(\mathscr{C} ; \mathbf{Q}) \rightarrow H_{\mathscr{G}}^{*}\left(\mathscr{C}_{\delta} ; \mathbf{Q}\right)
$$

is given by

$$
\begin{gathered}
a_{1} \mapsto a_{1}^{1}+a_{1}^{2}, \quad a_{2} \mapsto a_{1}^{1} a_{1}^{2}, \\
b_{1}^{j} \mapsto b_{1}^{1, j}+b_{1}^{2, j}, \quad b_{2} \mapsto a_{1}^{1} b_{1}^{2, j}+a_{1}^{2} b_{1}^{1, j},
\end{gathered}
$$

and

$$
f_{2} \mapsto \delta_{2} a_{1}^{1}+\delta_{1} a_{1}^{2}+\sum_{j=1}^{g} b_{1}^{1, j} b_{1}^{2, j+g}+b_{1}^{2, j} b_{1}^{1, j+g}
$$

Thus

$$
\left(\left(a_{1}\right)^{2}-4 a_{2}\right)^{g} \mapsto\left(a_{1}^{1}-a_{1}^{2}\right)^{2 g} .
$$

As before let $\beta$ denote the element $\left(a_{1}^{1}-a_{1}^{2}\right) / 2$ of $H_{\mathscr{G}}^{*}\left(\mathscr{C}_{\delta} ; \mathbf{Q}\right)$. Recall from the last section that in order to show $\left(\left(a_{1}\right)^{2}-4 a_{2}\right)^{g}$ is a relation in $H^{*}(\mathscr{M}(2, d) ; \mathbf{Q})$ it suffices to show that the image of $\left(\left(a_{1}\right)^{2}-4 a_{2}\right)^{g} \in H_{\mathscr{G}}^{*}(\mathscr{C} ; \mathbf{Q})$ under the direct sum of the restriction maps

$$
H_{\mathscr{G}}^{*}(\mathscr{C} ; \mathbf{Q}) \rightarrow \bigoplus_{\delta \in \Delta} H_{\mathscr{G}}^{*}\left(\mathscr{C}_{\delta} ; \mathbf{Q}\right)
$$

lies in the image in $\bigoplus_{\delta \in \Delta} H_{\mathscr{G}}^{*}\left(\mathscr{C}_{\delta} ; \mathbf{Q}\right)$ of the kernel of the restriction map

$$
H_{\mathscr{G}}^{*}(\mathscr{C} ; \mathbf{Q}) \rightarrow H_{\mathscr{G}}^{*}\left(\mathscr{C}^{\mathrm{ss}} ; \mathbf{Q}\right)
$$

Thus the Newstead-Ramanan conjecture follows from

Proposition 7. There is an element of the kernel of the restriction map

$$
H_{\mathscr{G}}^{*}(\mathscr{C} ; \mathbf{Q}) \rightarrow H_{\mathscr{G}}^{*}\left(\mathscr{C}^{\text {ss }} ; \mathbf{Q}\right)
$$

whose restriction to $H_{\mathscr{G}}^{*}\left(\mathscr{C}_{\delta} ; \mathbf{Q}\right)$ is $\beta^{2 g}$ for every $\delta \in \Delta$.

Proof. It was shown in $\S 2$ that there is a formal Laurent series $\Psi\left(x-a_{1}\right)$ in $x$ with coefficients in $H_{\mathscr{G}}^{*}(\mathscr{C} ; \mathbf{Q})$ such that if we express $\Psi\left(x-a_{1}\right)$ in the unique possible way as

$$
\sum_{S \subseteq\{1, \ldots, 2 g\}} \Psi_{S}(x) \prod_{j \in S} b_{1}^{j}
$$

where the coefficients of $\Psi_{S}(x)$ only involve $a_{1}, a_{2}, b_{2}^{j}-a_{1} b_{1}^{j} / 2$, and $f_{2}-$ $\sum_{1 \leq j \leq g} b_{1}^{j} b_{1}^{j+g}$, then the restriction to $H_{\mathscr{G}}^{*}\left(\mathscr{C}^{\text {ss }} ; \mathbf{Q}\right)$ of each $\Psi_{S}(x)$ is a polynomial in $x$. In particular, this implies that the restriction to $H_{\mathscr{G}}^{*}\left(\mathscr{C}^{\mathrm{ss}} ; \mathbf{Q}\right)$ 
of

$$
\Omega(x)=\Psi_{\{1, \ldots, 2 g\}}(x) \Psi_{\{1, \ldots, 2 g\}}(-x)
$$

is a polynomial ${ }^{5}$. This means that if we express the formal Laurent series $\Omega(x)$ in the form

$$
\sum_{r=-\infty}^{2 g-2}\left(\pi_{r} x+\rho_{r}\right)\left(x^{2}+a_{2}-\left(a_{1}\right)^{2} / 4\right)^{r}
$$

then the restrictions to $H_{\mathscr{G}}^{*}\left(\mathscr{C}^{\text {ss }} ; \mathbf{Q}\right)$ of $\pi_{r}$ and $\rho_{r}$ are zero when $r<0$.

Therefore, to complete the proof of the proposition it is enough to prove

Lemma 4. The restriction to $H_{\mathscr{G}}^{*}\left(\mathscr{C}_{\delta} ; \mathbf{Q}\right)$ of $\Omega(x)$ is

$$
(-1)^{g+1}(g !)^{-2} 2^{-2 g} x^{2 g} /\left(x^{2}-\beta^{2}\right)
$$

and the restriction of $(-1)^{g+1}(g !)^{2} 2^{2 g} \rho_{-1}$ is $\beta^{2 g}$ for every $\delta \in \Delta$.

Proof. It was shown in $\S 2$ that the restriction to $H_{\mathscr{G}}^{*}\left(\mathscr{C}_{\delta} ; \mathbf{Q}\right)$ of $\Psi\left(x-a_{1} / 2\right)$ is

$$
(x+\beta)^{g+D}(x-\beta)^{g-1-D} \exp \left(\frac{\xi_{1}}{x+\beta}+\frac{\xi_{2}}{x-\beta}\right)
$$

where $D=\left(\delta_{1}-\delta_{2}-1\right) / 2$ and $\xi_{i}=\sum_{1 \leq k \leq g} b_{1}^{i, k} b_{1}^{i, k+g}$ for $i=1,2$. Thus writing

$$
2 b_{1}^{1, k}=\left(b_{1}^{1, k}+b_{1}^{2, k}\right)+\left(b_{1}^{1, k}-b_{1}^{2, k}\right)
$$

and

we find that

$$
2 b_{1}^{2, k}=\left(b_{1}^{1, k}+b_{1}^{2, k}\right)-\left(b_{1}^{1, k}-b_{1}^{2, k}\right)
$$

$$
4 \xi_{1}=\xi+\hat{\xi}+\tilde{\xi}
$$

and

$$
4 \xi_{2}=\xi+\hat{\xi}-\tilde{\xi}
$$

where

$$
\xi=\sum_{1 \leq k \leq g}\left(b_{1}^{1, k}-b_{1}^{2, k}\right)\left(b_{1}^{1, k+g}-b_{1}^{2, k+g}\right)
$$

is defined as before and

$$
\hat{\xi}=\sum_{1 \leq k \leq g}\left(b_{1}^{1, k}+b_{1}^{2, k}\right)\left(b_{1}^{1, k+g}+b_{1}^{2, k+g}\right)
$$

and

$$
\tilde{\xi}=\sum_{1 \leq k \leq g}\left(b_{1}^{1, k}-b_{1}^{2, k}\right)\left(b_{1}^{1, k+g}+b_{1}^{2, k+g}\right)+\left(b_{1}^{1, k}+b_{1}^{2, k}\right)\left(b_{1}^{1, k+g}-b_{1}^{2, k+g}\right) .
$$

Therefore

$$
\frac{\xi_{1}}{x+\beta}+\frac{\xi_{2}}{x-\beta}=\frac{x(\xi+\hat{\xi})-\beta \tilde{\xi}}{2\left(x^{2}-\beta^{2}\right)}
$$

\footnotetext{
${ }^{5}$ Note that it makes sense to multiply the formal Laurent series $\Psi_{S}(x)$ together since the coefficients of $x^{j}$ with $j \geq 2 g$ in each $\Psi_{S}(x)$ are zero.
} 
Hence the restriction to $H_{\mathscr{G}}^{*}\left(\mathscr{C}_{\delta} ; \mathbf{Q}\right)$ of $\Psi\left(x-a_{1} / 2\right)$ is

$$
(x+\beta)^{g+D}(x-\beta)^{g-1-D} \exp \left(\frac{x \xi}{2\left(x^{2}-\beta^{2}\right)}\right) \exp \left(\frac{x \hat{\xi}}{2\left(x^{2}-\beta^{2}\right)}\right) \exp \left(\frac{-\beta \tilde{\xi}}{2\left(x^{2}-\beta^{2}\right)}\right) \text {. }
$$

The restriction to $H_{\mathscr{G}}^{*}\left(\mathscr{C}_{\delta} ; \mathbf{Q}\right)$ of the coefficient $\Psi_{S}(x)$ of $\prod_{j \in S} b_{1}^{j}$ in $\Psi\left(x-a_{1} / 2\right)$ for $S \subseteq\{1, \ldots, 2 g\}$ is obtained by expressing $\Psi\left(x-a_{1} / 2\right)$ in terms of $b_{1}^{1, j}+b_{1}^{2, j}, b_{1}^{1, j}-b_{1}^{2, j}$, and $\beta$, and then taking the coefficient of $\prod_{j \in S}\left(b_{1}^{1, j}+b_{1}^{2, j}\right)$ in this expression. Since the coefficient of $\prod_{j \in S}\left(b_{1}^{1, j}+b_{1}^{2, j}\right)$ in

$$
\exp \left(\frac{-\beta \tilde{\xi}}{2\left(x^{2}-\beta^{2}\right)}\right)
$$

is always zero if $S$ contains both $j$ and $j+g$ for any $j$, it follows that the restriction to $H_{\mathscr{G}}^{*}\left(\mathscr{C}_{\delta} ; \mathbf{Q}\right)$ of $\Psi_{\{1, \ldots, 2 g\}}(x)$ is

$$
(x+\beta)^{g+D}(x-\beta)^{g-1-D} \exp \left(\frac{x \xi}{2\left(x^{2}-\beta^{2}\right)}\right) \frac{x^{g}}{g ! 2^{g}\left(x^{2}-\beta^{2}\right)^{g}} .
$$

Thus the restriction of $\Omega(x)$ is

$$
(x+\beta)^{g+D}(-x+\beta)^{g+D}(x-\beta)^{g-1-D}(-x-\beta)^{g-1-D} \frac{x^{g}(-x)^{g}}{(g !)^{2} 2^{2 g}\left(x^{2}-\beta^{2}\right)^{2 g}},
$$

which simplifies to give

$$
(-1)^{g} \frac{x^{2 g}}{(g !)^{2} 2^{2 g}\left(x^{2}-\beta^{2}\right)}
$$

as required.

Finally to calculate the restriction to $H_{\mathscr{G}}^{*}\left(\mathscr{C}_{\delta} ; \mathbf{Q}\right)$ of $\rho_{-1}$ we write

$$
x^{2 g}=\left(x^{2}-\beta^{2}\right) q(x)+\eta x+\zeta
$$

and formally put $x=\beta$ and $x=-\beta$ to obtain $\eta=0$ and $\zeta=\beta^{2 g}$.

This completes the proof of the lemma and hence also of the proposition and the Newstead-Ramanan conjecture.

Remark 5. The conjecture of Newstead and Ramanan has been generalised to bundles of rank $n>2$ by Neeman [15], who conjectured that the Pontryagin ring of the tangent bundle to the moduli space $\mathscr{M}(2, d)$ should vanish in dimensions at least $2 g n^{2}-4 g(n-1)+2$ and proved that it does vanish in dimensions at least $5 g-3$ when $n=2$.

\section{RESOLVING SINGULARITIES}

In this section we shall describe a different approach to finding relations among the rational generators $a_{r}, b_{r}^{j}$, and $f_{r}$ for the cohomology ring of the moduli space $\mathscr{M}(n, d)$. This approach involves first resolving the singularities of the closure of the stratum $\mathscr{C}_{\mu}$ for each $\mu \in \mathscr{M}-\left\{\mu_{0}\right\}$. It is then possible 
to relate the Thom-Gysin map associated to the removal of $\mathscr{C}_{\mu}$ from the open subset $U_{\mu}$ of $\mathscr{C}$ to the Thom-Gysin map associated to the removal of the nonsingular closure $\tilde{\mathscr{C}}_{\mu}$ of $\mathscr{C}_{\mu}$ in an appropriate blow-up of $\mathscr{C}$.

This gives an approach to proving the Newstead-Ramanan conjecture that is possibly more intuitively appealing than the proof in the last section. A complete proof along these lines would be considerably more intricate than the one already given, so details will be omitted. However, it seems worthwhile to describe the main ideas, since these can be applied in more general circumstances as in $\S 6$ when no complete set of relations is known.

First we must consider what the closure of the stratum $\mathscr{C}_{\mu}$ looks like in a neighbourhood of $\mathscr{C}_{\delta}$ if $\delta>\mu$, i.e., if $\delta_{1}>d_{1}$ where $\delta=\left(\delta_{1}, \delta_{2}\right)$ and $\mu=\left(d_{1}, d_{2}\right)$.

Let us assume that $E$ is a bundle with canonical filtration $0 \subset L_{1} \subset E$ where $L_{1}$ and $L_{2}=E / L_{1}$ are line bundles of degrees $\delta_{1}>d_{1}$ and $\delta_{2}$. Then $E$ represents a point in $\mathscr{C}_{\delta}$. By deformation theory (cf. [2, p. 566] or [18, Chapter $2, \S 5])$ the vector space $H^{1}\left(M, L_{1}^{*} \otimes L_{2}\right)$ represents holomorphic deformations of $E$ modulo the deformations which respect the canonical filtration, and hence can be naturally identified with the normal space to $\mathscr{C}_{\delta}$ in $\mathscr{C}$ at this point.

Let $\Sigma^{j}(M)$ denote the $j$ th symmetric power of $M$ (i.e., the space of effective divisors $D$ of degree $j$ on $M$ ), which is nonsingular since $M$ has complex dimension one. For each $D \in \Sigma^{\delta_{1}-d_{1}}(M)$ let $L_{2}^{D}$ denote the sheaf which is the quotient of $E$ by the image of the map $L_{1} \otimes \mathscr{O}(-D) \rightarrow L_{1}$ defined by $D$. Then by deformation theory again the vector space

$$
H^{1}\left(M, L_{1}^{*} \otimes \mathscr{O}(D) \otimes L_{2}^{D}\right)
$$

represents holomorphic deformations of $E$ modulo the deformations which respect the image of the map $L_{1} \otimes \mathscr{O}(-D) \rightarrow L_{1}$. Now we have an exact sequence of sheaves

$$
0 \rightarrow \mathscr{O}_{D} \rightarrow L_{2}^{D} \rightarrow L_{2} \rightarrow 0
$$

where $\mathscr{O}_{D}$ is the skyscraper sheaf, which gives us an exact sequence

$$
0 \rightarrow \mathscr{O}_{D} \rightarrow L_{1}^{*} \otimes \mathscr{O}(D) \otimes L_{2}^{D} \rightarrow L_{1}^{*} \otimes \mathscr{O}(D) \otimes L_{2} \rightarrow 0
$$

and this induces an isomorphism

$$
H^{1}\left(M, L_{1}^{*} \otimes \mathscr{O}(D) \otimes L_{2}^{D}\right) \rightarrow H^{1}\left(M, L_{1}^{*} \otimes \mathscr{O}(D) \otimes L_{2}\right)
$$

Moreover, since the degree of $L_{1}^{*} \otimes \mathscr{O}(D) \otimes L_{2}$ is negative, the exact sequence

$$
0 \rightarrow L_{1}^{*} \otimes L_{2} \rightarrow L_{1}^{*} \otimes \mathscr{O}(D) \otimes L_{2} \rightarrow \mathscr{O}_{D} \rightarrow 0
$$

induces an exact sequence

$$
0 \rightarrow H^{0}\left(M, \mathscr{O}_{D}\right) \rightarrow H^{1}\left(M, L_{1}^{*} \otimes L_{2}\right) \rightarrow H^{1}\left(M, L_{1}^{*} \otimes \mathscr{O}(D) \otimes L_{2}\right) \rightarrow 0 .
$$

Hence there is a subbundle $\mathscr{H}$ of rank $\delta_{1}-d_{1}$ of the pullback to $\mathscr{C}_{\delta} \times$ $\Sigma^{\delta_{1}-d_{1}}(M)$ of the normal bundle to $\mathscr{C}_{\delta}$ in $\mathscr{C}$, whose fibre at a point 
represented by $E \in \mathscr{C}_{\delta}$ and $D \in \Sigma^{\delta_{1}-d_{1}}(M)$ is naturally isomorphic to $H^{0}\left(M, \mathscr{O}_{D}\right)$ embedded in $H^{1}\left(M, L_{1}^{*} \otimes L_{2}\right)$ as in this exact sequence.

If we identify the compact Riemann surface $M$ with a nonsingular complex projective curve we have the following results about how bundles degenerate from $\S 4$ of [17] (particularly Proposition 9); note that the definition of a vector bundle used by Shatz is more general than the one used in this paper, so his results have to be translated.

Let $S=\operatorname{Spec}(A)$ where $A$ is a discrete valuation ring, and let $\mathscr{E}$ be an algebraic vector bundle of rank two over $S \times M$. Thus $\mathscr{E}$ defines an algebraic family $\left\{\mathscr{E}_{x}: x \in S\right\}$ of vector bundles over $S$ by $\mathscr{E}_{x}=\left.\mathscr{E}\right|_{\{x\} \times M}$. Let $s_{0}$ and $S$ denote the special and generic points of $S$, and suppose that $E=\mathscr{E}_{S_{0}}$ is a bundle over $M$ with canonical filtration $0 \subset L_{1} \subset E$ where $L_{1}$ and $L_{2}=E / L_{1}$ are line bundles of degrees $\delta_{1}$ and $\delta_{2}$. Suppose also that $L$ is a line subbundle of the bundle $\mathscr{E}_{s}$ of degree $d_{1}$, so that $0 \subset L \subset \mathscr{E}_{s}$ is the canonical filtration of $\mathscr{E}_{s}$. Then this line subbundle extends canonically to a subsheaf $\mathscr{L}$ of $\mathscr{E}$ such that there is a nonempty open subset of $S \times M$ containing all points of codimension at most one on which the subsheaf $\mathscr{L}$ and the quotient $\mathscr{E} / \mathscr{L}$ are locally free (i.e., the restriction of $\mathscr{L}$ to this open subset is a subbundle in our sense) and such that the restriction of $\mathscr{L}$ to $\left\{s_{0}\right\} \times M$ has the same degree and rank as $\mathscr{L}_{s}=L$ for each $j$. Since $d_{1}>d / 2>\delta_{2}$ and there are no nonzero holomorphic maps from line bundles of degree strictly greater than $\delta_{2}$ to $L_{2}$, it follows that the restriction of $\mathscr{L}$ to $\left\{s_{0}\right\} \times M$ is the image of the map of line bundles $L_{1} \otimes \mathscr{O}(-D) \rightarrow L_{1}$ defined by some effective divisor $D$ of degree $\delta_{1}-d_{1}$ on $M \times\left\{s_{0}\right\}$. Note that the divisor $D$ is uniquely determined by the family $\mathscr{E}$ of bundles. Moreover, $\mathscr{E}_{s}$ determines a deformation of $E$ which respects the map $L_{1} \otimes \mathscr{O}(-D) \rightarrow E$ but not the map $L_{1} \rightarrow E$, and hence determines an element of the projective space associated to the kernel of the map

$$
H^{1}\left(M, L_{1}^{*} \otimes L_{2}\right) \rightarrow H^{1}\left(M, L_{1}^{*} \otimes \mathscr{O}(D) \otimes L_{2}\right)
$$

or, equivalently, a point of the projective bundle $\mathbf{P}(\mathscr{H})$ over $\mathscr{C}_{\delta} \times \Sigma^{\delta_{1}-d_{1}}(M)$.

Remark 6. We can see explicitly, using transition functions, how bundles of type $\mu=\left(d_{1}, d_{2}\right)$ can degenerate to bundles of type $\delta=\left(\delta_{1}, \delta_{2}\right)$ when $\delta>\mu$. Let us suppose that $L_{1} \oplus L_{2}$ is a holomorphic bundle of type $\delta$ (a similar argument applies if we replace $L_{1} \oplus L_{2}$ by a general bundle of type $\delta$, not necessarily a sum of two line bundles) and let $D=m_{1} x_{1}+\cdots+m_{k} x_{k}$ be an effective divisor of degree $\delta_{1}-d_{1}$ on $M$. Choose an open cover $\left\{U_{1}, \ldots, U_{\ell}\right\}$ of $M$ with $\ell \geq$ $k$ such that $U_{1}, \ldots, U_{k}$ are disjoint open neighbourhoods of $x_{1}, \ldots, x_{k}$ and the line bundles $L_{1}$ and $L_{2}$ can be trivialised over the open sets $U_{1}, \ldots, U_{\ell}$ with transition functions $\alpha_{i, j}$ and $\beta_{i, j}$ on $U_{i} \cap U_{j}$. Choose holomorphic local coordinates vanishing at $x_{1}, \ldots, x_{k}$ on neighbourhoods $V_{1}, \ldots, V_{k}$ of 
$x_{1}, \ldots, x_{k}$ such that $\bar{V}_{j} \subseteq U_{j}$. Let

$$
W_{j}= \begin{cases}U_{j}-\left\{x_{j}\right\} & \text { if } 1 \leq j \leq k \\ U_{j}-\left(\overline{V_{1}} \cup \cdots \cup \overline{V_{k}}\right) & \text { if } k<j \leq \ell .\end{cases}
$$

Then $\left\{V_{1}, \ldots, V_{k}, W_{1}, \ldots, W_{\ell}\right\}$ is an open cover of $M$ such that $V_{i} \cap W_{j}=\emptyset$ if $i \neq j$ and $V_{i} \cap V_{j}=\emptyset=W_{i} \cap W_{j}$ if $1 \leq i<j \leq k$.

Now let $\mathscr{E}$ be the holomorphic bundle of rank two over $\mathbf{C} \times M$ defined with respect to the open cover $\left\{\mathbf{C} \times V_{1}, \ldots, \mathbf{C} \times V_{k}, \mathbf{C} \times W_{1}, \ldots, \mathbf{C} \times W_{\ell}\right\}$ by transition functions

$$
\left(\begin{array}{cc}
\alpha_{i, j} & 0 \\
0 & \beta_{i, j}
\end{array}\right)
$$

on $\mathbf{C} \times\left(W_{i} \cap W_{j}\right)$ if $i<j$ and $j>k$ and

$$
\left(\begin{array}{cc}
1 & t z^{-m_{j}} \\
0 & 1
\end{array}\right)
$$

on the product of $\mathbf{C}$ with $V_{j} \cap W_{j}=V_{j}-\left\{x_{j}\right\}$, where $z$ is the chosen local coordinate on $V_{j}$ vanishing at $x_{j}$ and $t$ parametrises $\mathbf{C}$. Then $\left\{\mathscr{E}_{t}=\left.\mathscr{E}\right|_{\{t\} \times M}\right.$ : $t \in \mathbf{C}\}$ is a family of holomorphic bundles of rank two and degree $d$ over $M$ such that $\mathscr{E}_{0}=L_{1} \oplus L_{2}$.

The line bundle $L_{1} \otimes \mathscr{O}(-D)$ can be trivialised over the same cover

$$
\left\{V_{1}, \ldots, V_{k}, W_{1}, \ldots, W_{\ell}\right\}
$$

of $M$ with transition functions $\alpha_{i, j}$ on $W_{i} \cap W_{j}$ if $i<j$ and $j>k$ and $z^{-m_{j}}$ on $V_{j} \cap W_{j}$. If $t \neq 0$ there is an embedding of $L_{1} \otimes \mathscr{O}(-D)$ into $\mathscr{E}_{t}$ defined with respect to this trivialisation by the $1 \times 2$ matrices $\left(z^{m_{j}},-t\right)$ over $V_{j}$ and $(1,0)$ over $W_{j}$. Since the degree of $L_{1} \otimes \mathscr{O}(-D)$ is $d_{1}$, this implies that $\mathscr{E}_{t}$ is of type $\mu$ when $t \neq 0$.

Recall that the fibre at $\left(L_{1} \oplus L_{2}, D\right)$ of the projective bundle $\mathbf{P}(\mathscr{H})$ can be identified with $\mathbf{P}\left(H^{0}\left(M, \mathscr{O}_{D}\right)\right)$. The point of this projective space determined by the family of bundles $\mathscr{E}$ as above depends on the choice of holomorphic local coordinates $z$ near the support of $D$. We can obtain different families by replacing each term $z^{-m_{j}}$ by the principal part

$$
a_{-1} z^{-1}+\cdots+a_{-m_{j}} z^{-m_{j}}
$$

of $\phi(z)^{-m_{j}}$ where $\phi(z)$ is any other local coordinate near $x_{j}$ vanishing at $x_{j}$, and then the associated points of $\mathbf{P}\left(H^{0}\left(M, \mathscr{O}_{D}\right)\right)$ are all those points which do not belong to $\mathbf{P}\left(H^{0}\left(M, \mathscr{O}_{D^{\prime}}\right)\right)$ for any effective divisor $D^{\prime}<D$.

Now consider the result of blowing up $U_{\delta}=\mathscr{C}-\bigcup_{\gamma>\delta} \mathscr{C}_{\gamma}$ along $\mathscr{C}_{\delta}$. This is possible since $\mathscr{C}_{\delta}$ is a closed complex submanifold of $U_{\delta}$ whose codimension is finite. 
Lemma 5. The intersection of the exceptional divisor with the closure of the stratum $\mathscr{C}_{\mu}$ in this blow-up of $U_{\delta}$ can be identified with the image of the projective bundle $\mathbf{P}(\mathscr{H})$ over $\mathscr{C}_{\delta} \times \Sigma^{\delta_{1}-d_{1}}(M)$ in the projective normal bundle to $\mathscr{C}_{\delta}$. If $\delta_{1}=d_{1}+1$ then the closure of the stratum $\mathscr{C}_{\mu}$ is nonsingular and its intersection with the exceptional divisor can be identified with $\mathscr{C}_{\delta} \times M$.

Proof. Recall that $\mathscr{H}$ is a subbundle of rank $\delta_{1}-d_{1}$ of the pullback to $\mathscr{C}_{\delta} \times$ $\Sigma^{\delta_{1}-d_{1}}(M)$ of the normal bundle to $\mathscr{C}_{\delta}$ in $\mathscr{C}$, whose fibre at a point represented by $E \in \mathscr{C}_{\delta}$ and $D \in \Sigma^{\delta_{1}-d_{1}}(M)$ is naturally isomorphic to $H^{0}\left(M, \mathscr{O}_{D}\right)$ embedded in $H^{1}\left(M, L_{1}^{*} \otimes L_{2}\right)$ as in the exact sequence

$$
0 \rightarrow H^{0}\left(M, \mathscr{O}_{D}\right) \rightarrow H^{1}\left(M, L_{1}^{*} \otimes L_{2}\right) \rightarrow H^{1}\left(M, L_{1}^{*} \otimes \mathscr{O}(D) \otimes L_{2}\right) \rightarrow 0 .
$$

Thus there is a natural map from $\mathscr{H}$ to the normal bundle to $\mathscr{C}_{\delta}$ in $\mathscr{C}$, which collapses the zero section over $\mathscr{C}_{\delta} \times \Sigma^{\delta_{1}-d_{1}}(M)$ to the zero section over $\mathscr{C}_{\delta}$. This map induces a map from $\mathbf{P}(\mathscr{H})$ to the exceptional divisor when the exceptional divisor is identified in the natural way with the projective normal bundle to $\mathscr{C}_{\delta}$ in $\mathscr{C}$. From the discussion above of families of bundles over discrete valuation rings, together with property (i) of the stratification from $\S 1$ and Grothendieck's valuative criterion for properness (see [6, Chapter II, Theorem 4.7 and Exercise 4.11]), it follows that the union of the image of this map with $\bigcup_{\mu \leq \gamma<\delta} \mathscr{C}_{\gamma}$ is closed in the blow-up of $U_{\delta}$. Conversely the last remark shows that any element of the image of $\mathbf{P}(\mathscr{H})$ belongs to the closure of $\mathscr{C}_{\mu}$ in the blow-up. Locally, near the complement in the exceptional divisor of the closure of $\bigcup_{\mu<\gamma<\delta} \mathscr{C}_{\gamma}$, the closure of $\mathscr{C}_{\mu}$ in the blow-up looks like the tautological line bundle over $\mathbf{P}(\mathscr{H})$ and hence is nonsingular.

When $\delta_{1}=d_{1}+1$ then $\Sigma^{\delta_{1}-d_{1}}(M)=M$ and $\mathscr{H}$ is a line bundle, so the projective bundle $\mathrm{P}(\mathscr{H})$ is just $\mathscr{C}_{\delta} \times M$ and the result follows.

Next consider the result of blowing up $U_{\delta}=\mathscr{C}-\bigcup_{\gamma>\delta} \mathscr{C}_{\gamma}$ along first $\mathscr{C}_{\delta}$ and then successively along the closures of the strata $\mathscr{C}_{\gamma}$ with $\mu<\gamma<\delta$. In fact, because the stratification of $\mathscr{C}$ is locally finite, we can apply the same process to the whole of $\mathscr{C}$ to obtain a blow-up $\pi: \widetilde{\mathscr{C}} \rightarrow \mathscr{C}$ of $\mathscr{C}$ successively along all the strata $\mathscr{C}_{\gamma}$ with $\gamma>\mu$. (Note that $\widetilde{\mathscr{C}}$ depends on $\mu$.)

Let $\widetilde{\mathscr{C}}_{\mu}$ denote the closure of $\mathscr{C}_{\mu}$ in $\widetilde{\mathscr{C}}$, and let $\mathscr{E}_{\delta}$ denote the inverse image of $\mathscr{C}_{\delta}$ in $\tilde{\mathscr{C}}$ if $\delta>\mu$.

Lemma 6. The closure $\tilde{\mathscr{C}}_{\mu}$ in $\widetilde{\mathscr{C}}$ of the stratum $\mathscr{C}_{\mu}$ is nonsingular. Its intersection with the inverse image of $\mathscr{C}_{\delta}$ can be identified with a blow-up of the projective bundle $\mathbf{P}(\mathscr{H})$ over $\mathscr{C}_{\delta} \times \Sigma^{\delta_{1}-d_{1}}(M)$.

Proof. This follows from the proof of the previous lemma. 
$\tilde{\mathscr{C}}_{\mu}$ is the disjoint union of $\mathscr{C}_{\mu}$ together with all the subsets

$$
\mathscr{E}_{\left[\gamma_{1}, \ldots, \gamma_{k}\right]}=\widetilde{\mathscr{C}}_{\mu} \cap\left(\mathscr{E}_{\gamma_{1}} \cap \cdots \cap \mathscr{E}_{\gamma_{k}}-\bigcup_{\mu<\gamma \notin\left\{\gamma_{1}, \ldots, \gamma_{k}\right\}} \mathscr{E}_{\gamma}\right)
$$

for $\gamma_{1}, \ldots, \gamma_{k} \in \mathscr{M}$ satisfying $\mu<\gamma_{1}<\cdots<\gamma_{k}$. Moreover, if we set $\gamma_{0}=\mu$ and write $\gamma_{j}=\left(\gamma_{1}^{j}, \gamma_{2}^{j}\right)$ when $0 \leq j \leq k$ and $\ell_{j}=\gamma_{1}^{j}-\gamma_{1}^{j-1}$ when $1 \leq j \leq k$, then $\mathscr{E}_{\left[\gamma_{1}, \ldots, \gamma_{k}\right]}$ can be identified with a product

$$
Q_{1} \times \cdots \times Q_{k} \times \mathscr{C}_{\gamma_{k}}
$$

where $Q_{j}$ fibres over the symmetric power $\Sigma^{\ell_{j}}(M)$ of $M$ with the fibre over $D \in \Sigma^{\ell_{j}}(M)$ given by

$$
\mathbf{P}\left(H^{0}\left(M, \mathscr{O}_{D}\right)\right)-\bigcup_{D^{\prime} \in \Sigma^{\ell_{j}-1}(M), D^{\prime}<D} \mathbf{P}\left(H^{0}\left(M, \mathscr{O}_{D^{\prime}}\right)\right)
$$

With respect to this identification the restriction to $\mathscr{E}_{\left[\gamma_{1}, \ldots, \gamma_{k}\right]}$ of $\pi: \widetilde{\mathscr{C}} \rightarrow \mathscr{C}$ is projection onto $\mathscr{C}_{\gamma_{k}}$.

Recall that the generators $a_{1}^{i}$ and $b_{1}^{i, j}$ of $H_{\mathscr{G}}^{*}\left(\mathscr{C}_{\mu} ; \mathbf{Q}\right)$ are obtained by applying the Kunneth decomposition to the equivariant Chern classes of the universal line bundles $\mathscr{V}_{1}$ and $\mathscr{V}_{2}$ on $\mathscr{C}_{\mu} \times M$ whose restrictions to $\{p\} \times M$ are canonically isomorphic to the line bundles $L_{1}$ and $L_{2}$, where $p \in \mathscr{C}_{\mu}$ represents a bundle $E$ of type $\mu$ over $M$ with canonical filtration $0 \subset L_{1} \subset E$ and $L_{2}=E / L_{1}$. It can be shown, using the description of how bundles of type $\delta$ degenerate to bundles of type $\mu$ given above, that these universal line bundles extend to line bundles $\tilde{\mathscr{V}}_{1}$ and $\tilde{\mathscr{V}}_{2}$ on $\tilde{\mathscr{C}}_{\mu} \times M$ such that $\tilde{\mathscr{V}}_{1} \otimes \tilde{\mathscr{V}}_{2}$ is the pullback of the determinant of the universal bundle on $\mathscr{C} \times M$ and such that the fibre of the normal bundle to $\widetilde{\mathscr{C}}_{\mu}$ in $\widetilde{\mathscr{C}}$ at a point $x$ is naturally isomorphic to

$$
H^{1}\left(M,\left.\tilde{\mathscr{V}}_{1}^{*} \otimes \tilde{\mathscr{V}}_{2}\right|_{\{x\} \times M}\right) \text {. }
$$

By decomposing the first Chern class of $\tilde{\mathscr{V}}_{1}$ and $f \widetilde{\mathscr{V}}_{2}$ according to the Künneth decomposition of $H^{2}\left(\tilde{\mathscr{C}}_{\mu} \times M ; \mathbf{Q}\right)$ we find that the generators $a_{1}^{i}$ and $b_{1}^{i, j}$ for $1 \leq i \leq 2,1 \leq j \leq 2 g$ of $H_{\mathscr{G}}^{*}\left(\mathscr{C}_{\mu} ; \mathbf{Q}\right)$ lift to elements $\tilde{a}_{1}^{i}$ and $\tilde{b}_{1}^{i, j}$, say, of $H_{\mathscr{G}}^{*}\left(\tilde{\mathscr{C}}_{\mu} ; \mathbf{Q}\right)$. It then follows by a trivial modification of the proof of Lemma 2 in $\S 1$ that the equivariant Euler class of the normal bundle to $\tilde{\mathscr{C}}_{\mu}$ in $\tilde{\mathscr{C}}$ is

$$
\left(\tilde{a}_{1}^{2}-\tilde{a}_{1}^{1}\right)^{d_{1}-d_{2}-1}\left(\left(\tilde{a}_{1}^{2}-\tilde{a}_{1}^{1}\right)^{g}-\tilde{\xi}\left(\tilde{a}_{1}^{2}-\tilde{a}_{1}^{1}\right)^{g-1}+\ldots+(-\tilde{\xi})^{g} / g !\right)
$$

where

$$
\tilde{\xi}=\sum_{1 \leq j \leq g}\left(b_{1}^{1, j}-b_{1}^{2, j}\right)\left(b_{1}^{1, j+g}-b_{1}^{2, j+g}\right) .
$$


Moreover, $(\tilde{\xi})^{g+1}=0$ so we can also write this as

$$
\left(\tilde{a}_{1}^{2}-\tilde{a}_{1}^{1}\right)^{g+d_{1}-d_{2}-1} \exp \left(-\tilde{\xi} /\left(\tilde{a}_{1}^{2}-\tilde{a}_{1}^{1}\right) .\right.
$$

This means that if as before $\mu_{1}=((d+1) / 2,(d-1) / 2)$ then the restriction to $\tilde{\mathscr{C}}_{\mu_{1}}$ of the image under the Thom-Gysin map

$$
H_{\mathscr{G}}^{*-2 d\left(\mu_{1}\right)}\left(\tilde{\mathscr{C}}_{\mu_{1}} ; \mathbf{Q}\right) \rightarrow H_{\mathscr{G}}^{*}(\tilde{\mathscr{C}} ; \mathbf{Q})
$$

of the lift

$$
\left(\tilde{a}_{1}^{2}-\tilde{a}_{1}^{1}\right)^{g} \exp \left(\tilde{\xi} /\left(\tilde{a}_{1}^{2}-\tilde{a}_{1}^{1}\right)\right)
$$

of the element

$$
\left(a_{1}^{2}-a_{1}^{1}\right)^{g} \exp \left(\xi /\left(a_{1}^{2}-a_{1}^{1}\right)\right)
$$

of $H_{\mathscr{G}}^{*}\left(\mathscr{C}_{\mu_{1}} ; \mathbf{Q}\right)$ is $\left(\tilde{a}_{1}^{2}-\tilde{a}_{1}^{1}\right)^{2 g}$. If we compare the Thom-Gysin sequences

$$
\cdots \rightarrow H_{\mathscr{G}}^{*-2 d(\mu)}\left(\mathscr{C}_{\mu} ; \mathbf{Q}\right) \rightarrow H_{\mathscr{G}}^{*}\left(U_{\mu} ; \mathbf{Q}\right) \rightarrow H_{\mathscr{G}}^{*}\left(U_{\mu}-\mathscr{C}_{\mu} ; \mathbf{Q}\right) \rightarrow \cdots
$$

and

$$
\cdots \rightarrow H_{\mathscr{G}}^{*-2 d(\mu)}\left(\tilde{\mathscr{C}}_{\mu} ; \mathbf{Q}\right) \rightarrow H_{\mathscr{G}}^{*}(\tilde{\mathscr{C}} ; \mathbf{Q}) \rightarrow H_{\mathscr{G}}^{*}\left(\tilde{\mathscr{C}}-\tilde{\mathscr{C}}_{\mu} ; \mathbf{Q}\right) \rightarrow \cdots
$$

it is possible (but not very straightforward) to deduce from this that there is a lift

$$
H_{\mathscr{G}}^{*-2 d\left(\mu_{1}\right)}\left(\mathscr{C}_{\mu_{1}} ; \mathbf{Q}\right) \rightarrow H_{\mathscr{G}}^{*}(\mathscr{C} ; \mathbf{Q})
$$

of the Thom-Gysin map

$$
H_{\mathscr{G}}^{*-2 d\left(\mu_{1}\right)}\left(\mathscr{C}_{\mu_{1}} ; \mathbf{Q}\right) \rightarrow H_{\mathscr{G}}^{*}\left(U_{\mu_{1}} ; \mathbf{Q}\right)
$$

whose composition with the restriction map

$$
H_{\mathscr{G}}^{*}(\mathscr{C} ; \mathbf{Q}) \rightarrow H_{\mathscr{G}}^{*}\left(\mathscr{C}_{\delta} ; \mathbf{Q}\right)
$$

takes $\left(a_{1}^{2}-a_{1}^{1}\right)^{g} \exp \left(\xi /\left(a_{1}^{2}-a_{1}^{1}\right)\right)$ to $\left(a_{1}^{2}-a_{1}^{1}\right)^{2 g}$. Since the image of any such lift of this Thom-Gysin map is contained in the kernel of the restriction map

$$
H_{\mathscr{G}}^{*}(\mathscr{C} ; \mathbf{Q}) \rightarrow H_{\mathscr{G}}^{*}\left(\mathscr{C}^{\text {ss }} ; \mathbf{Q}\right)
$$

this would give an alternative proof of the proposition in $\S 4$ and hence of the Newstead-Ramanan conjecture.

\section{Generators and Relations}

FOR GEOMETRIC INVARIANT THEORETIC QUOTIENTS

Let $X$ be a nonsingular complex projective variety and let $G$ be a complex reductive group acting on $X$. Let us assume that $X$ is embedded in the complex projective space $\mathbf{P}_{n}$ and that the action of $G$ on $X$ extends to a linear action on $\mathbf{P}_{n}$ defined by a homomorphism $\rho: G \rightarrow \mathrm{GL}(n+1 ; \mathbf{C})$. Since $G$ is reductive, it is the complexification of a maximal compact subgroup $K$. Without loss of generality we may suppose that $K$ acts unitarily on $\mathbf{P}_{n}$ and thus preserves the Fubini-Study metric. 
The quotient of $X$ by this linear action of $G$ in the sense of geometric invariant theory [14] will be denoted by $X / / G$ to distinguish it from the ordinary topological quotient $X / G$. Let $X^{\mathrm{s}}$ and $X^{\text {ss }}$ be the open subsets of $X$ consisting of the stable ${ }^{6}$ and semistable points of $X$ under the action. Then there is a surjective $G$-invariant morphism $\phi: X^{\text {ss }} \rightarrow X / / G$ and any fibre of $\phi$ which meets $X^{\mathrm{s}}$ is a single $G$-orbit of dimension equal to that of $G$.

We shall assume that $X$ is connected and

$$
X^{\text {ss }}=X^{\mathrm{s}} \neq \emptyset \text {. }
$$

Then the stabiliser in $G$ of every $x \in X^{\text {ss }}$ is finite, $X / / G$ is topologically the ordinary quotient $X^{\mathrm{ss}} / G$, and there is a natural isomorphism

$$
H^{*}(X / / G ; \mathbf{Q}) \cong H_{G}^{*}\left(X^{\text {ss }} ; \mathbf{Q}\right)
$$

(see $[8,5.6$ and 7.5$])$. Moreover, by $[8,5.4$ and 8.10$]$ the restriction map

$$
H_{G}^{*}(X ; \mathbf{Q}) \rightarrow H_{G}^{*}\left(X^{\mathrm{ss}} ; \mathbf{Q}\right)
$$

is surjective, and by $[8,5.8]$ the spectral sequence associated to the fibration

$$
X \times{ }_{G} E G \rightarrow B G
$$

with fibre $X$ degenerates, so that

$$
H_{G}^{*}(X ; \mathbf{Q}) \cong H^{*}(X ; \mathbf{Q}) \otimes H^{*}(B G ; \mathbf{Q}) .
$$

Note that this isomorphism is an isomorphism of vector spaces, not necessarily of rings, but that nonetheless generators of the cohomology rings $H^{*}(X ; \mathbf{Q})$ and $H^{*}(B G ; \mathbf{Q})$ induce generators of $H_{G}^{*}(X ; \mathbf{Q})$ and thus of $H_{G}^{*}\left(X^{\text {ss }} ; \mathbf{Q}\right)$ or, equivalently, of the rational cohomology $H^{*}(X / / G ; \mathbf{Q})$ of the quotient $X / / G$.

The aim of this section is to describe the relations among these generators of $H^{*}(X / / G ; \mathbf{Q})$ in such a way that they can be determined in explicit examples. This problem amounts to determining the kernel of the restriction map $H_{G}^{*}(X ; \mathbf{Q}) \rightarrow H_{G}^{*}\left(X^{\mathrm{ss}} ; \mathbf{Q}\right)$. This will be done by first showing that if $T$ is a maximal torus of $K$ and $X^{T}$ is its fixed point set in $X$ then the restriction map $H_{G}^{*}(X ; \mathbf{Q}) \rightarrow H_{T}^{*}\left(X^{T} ; \mathbf{Q}\right)$ is injective, and then by describing the image of the kernel under this map (see Proposition 8 and Corollaries 4 and 5).

In [8] there is defined a stratification $\left\{S_{\beta} \mid \beta \in \mathscr{B}\right\}$ of $X$, indexed by a finite partially ordered set $\mathscr{B}$, which has the following useful properties:

(i) $X$ is the disjoint union of the strata $S_{\beta}$, and each stratum $S_{\beta}$ is a $G$ invariant, locally closed, nonsingular subvariety of $X$ such that $\overline{S_{\beta}} \subseteq \bigcup_{\gamma \geq \beta} S_{\gamma}$ where $\geq$ is the partial order on $\mathscr{B}$.

(ii) $X^{\text {ss }}$ coincides with the open stratum $S_{0}$.

\footnotetext{
${ }^{6}$ We shall follow the usual convention nowadays that the term stable corresponds to the term properly stable in [14].
} 
(iii) If $\beta \neq 0$ there is a proper nonsingular subvariety $Z_{\beta}$ of $X$ acted on by a reductive subgroup $\operatorname{Stab} \beta$ of $G$ such that

$$
H_{G}^{*}\left(S_{\beta} ; \mathbf{Q}\right) \cong H_{\text {Stab } \beta}^{*}\left(Z_{\beta}^{\text {ss }} ; \mathbf{Q}\right)
$$

where $Z_{\beta}^{\text {ss }}$ is the set of semistable points in $Z_{\beta}$ with respect to the action of $\operatorname{Stab} \beta$ appropriately linearised.

(iv) The stratification $\left\{S_{\beta} \mid \beta \in \mathscr{B}\right\}$ is equivariantly perfect over the rationals so that

$$
\begin{aligned}
H_{G}^{i}(X ; \mathbf{Q}) & \cong \bigoplus_{\beta \in \mathscr{B}} H_{G}^{i-2 \lambda(\beta)}\left(S_{\beta} ; \mathbf{Q}\right) \\
& =H_{G}^{i}\left(X^{\mathrm{ss}} ; \mathbf{Q}\right) \oplus \bigoplus_{\beta \neq 0} H_{G}^{i-2 \lambda(\beta)}\left(S_{\beta} ; \mathbf{Q}\right)
\end{aligned}
$$

for all $i$, where $\lambda(\beta)$ is the complex codimension of $S_{\beta}$ in $X$, and the restriction map $H_{G}^{*}(X ; \mathbf{Q}) \rightarrow H_{G}^{*}\left(X^{\mathrm{ss}} ; \mathbf{Q}\right)$ corresponds under this isomorphism to the projection onto the summand $H_{G}^{*}\left(X^{\mathrm{ss}} ; \mathbf{Q}\right)$. The isomorphism is one of vector spaces, not one of rings. Note that if a stratum $S_{\beta}$ is not connected then its codimension $\lambda(\beta)$ may not be well defined. In that case, $H_{G}^{i-2 \lambda(\beta)}\left(S_{\beta} ; \mathbf{Q}\right)$ must be interpreted as

$$
\bigoplus_{1 \leq j \leq k} H_{G}^{i-2 \lambda(\beta, j)}\left(S_{\beta, j} ; \mathbf{Q}\right)
$$

where $S_{\beta, 1}, \ldots, S_{\beta, k}$ are the connected components of $S_{\beta}$ and $\lambda(\beta, j)$ is the complex codimension of $S_{\beta, j}$.

The stratification $\left\{S_{\beta} \mid \beta \in \mathscr{B}\right\}$ of $X$ can be defined as the Morse stratification associated to a function $f: X \rightarrow \mathbf{R}$, in the sense that the set of critical points for $f$ in $X$ is a disjoint union of closed subsets $\left\{C_{\beta} \mid \beta \in \mathscr{B}\right\}$ of $X$ and a point $x \in X$ lies in the stratum $S_{\beta}$ if and only if its path of steepest descent under $f$ with respect to the Fubini-Study metric has a limit point in $C_{\beta}$. However, $f$ is not a Morse function in the classical sense, and the subsets $C_{\beta}$ of $X$ are not necessarily submanifolds (see [8]).

The function $f$ is defined as the norm-square $f=\|\mu\|^{2}$, taken with respect to a fixed invariant inner product on the Lie algebra $\mathbf{k}$ of the maximal compact subgroup $K$ of $G$, of the momentum map $\mu: X \rightarrow \mathbf{k}^{*}$ defined in homogeneous coordinates by

for all $a \in \mathbf{k}$ (see $[8,2.5]$ ).

$$
\mu(x) \cdot a=\frac{x a \bar{x}^{t}}{2 \pi i\|x\|^{2}}
$$

The indexing set $\mathscr{B}$ is a finite set of points in a positive Weyl chamber $\mathbf{t}_{+}$ in the Lie algebra $\mathbf{t}$ of a maximal torus $T$ of $K$. Let $\alpha_{0}, \ldots, \alpha_{n} \in \mathbf{t}^{*}$ be the weights of the representation of $T$ defining its action on $X$, and identify $\mathbf{t}^{*}$ with $\mathbf{t}$ using the fixed inner product. Then $\beta \in \mathbf{t}_{+}$belongs to $\mathscr{B}$ if and only if $\beta$ is the closest point to 0 of the convex hull in $\mathbf{t}$ of some nonempty subset of 
$\left\{\alpha_{0}, \ldots, \alpha_{n}\right\}$. If $\beta \in \mathscr{B}$ then $\operatorname{Stab} \beta$ is the stabiliser of $\beta$ under the adjoint action of $G$ and $Z_{\beta}$ is the intersection of $X$ with the linear subspace

$$
\left\{\left(x_{0} ; \ldots ; x_{n}\right) \mid x_{i}=0 \text { if } \alpha_{i} \cdot \beta \neq\|\beta\|^{2}\right\}
$$

of $\mathbf{P}_{n}$. It is a union of connected components of the fixed point set of the subtorus $T_{\beta}$ of $T$ generated by $\beta$. Also

$$
S_{\beta}=G Y_{\beta}=G \overline{Y_{\beta}}-\bigcup_{\|\gamma\|>\|\beta\|} G \overline{Y_{\gamma}}
$$

where

$$
\overline{Y_{\beta}}=\left\{\left(x_{0} ; \ldots ; x_{n}\right) \mid x_{i}=0 \text { if } \alpha_{i} \cdot \beta<\|\beta\|^{2}\right\}
$$

and

$$
Y_{\beta}=\left\{\left(x_{0} ; \ldots ; x_{n}\right) \in \overline{Y_{\beta}} \mid x_{i} \neq 0 \text { for some } i \text { such that } \alpha_{i} . \beta=\|\beta\|^{2}\right\} .
$$

It follows from property (iv) of the stratification $\left\{S_{\beta} \mid \beta \in \mathscr{B}\right\}$ that the kernel of the restriction map $H_{G}^{*}(X ; \mathbf{Q}) \rightarrow H_{G}^{*}\left(X^{\text {ss }} ; \mathbf{Q}\right)$ is isomorphic as a vector space to the direct sum

$$
\bigoplus_{\beta \neq 0} H_{G}^{i-2 \lambda(\beta)}\left(S_{\beta} ; \mathbf{Q}\right)
$$

(This gives one an inductive formula for computing the equivariant Betti numbers of $X^{\text {ss }}$ and thus the Betti numbers of the quotient $X / / G$; see [8, 8.12]). However, our aim is to describe the kernel as a subset of $H_{G}^{*}(X ; \mathbf{Q})$. If $\beta \in \mathscr{B}$ then by property (i) of the stratification, the subset

$$
U_{\beta}=X-\bigcup_{\gamma>\beta} S_{\gamma}=\bigcup_{\gamma \ngtr \beta} S_{\gamma}
$$

is an open subset of $X$ containing $S_{\beta}$ as a closed submanifold. Thus there is a long exact sequence (the Thom-Gysin sequence)

$$
\cdots \rightarrow H_{G}^{j-2 \lambda(\beta)}\left(S_{\beta} ; \mathbf{Q}\right) \rightarrow H_{G}^{j}\left(U_{\beta} ; \mathbf{Q}\right) \rightarrow H_{G}^{j}\left(U_{\beta}-S_{\beta} ; \mathbf{Q}\right) \rightarrow \cdots .
$$

To say that the stratification is equivariantly perfect over the rationals means that all these long exact sequences break up into short exact sequences

$$
0 \rightarrow H_{G}^{j-2 \lambda(\beta)}\left(S_{\beta} ; \mathbf{Q}\right) \rightarrow H_{G}^{j}\left(U_{\beta} ; \mathbf{Q}\right) \rightarrow H_{G}^{j}\left(U_{\beta}-S_{\beta} ; \mathbf{Q}\right) \rightarrow 0 .
$$

In particular, this implies that the restriction maps $H_{G}^{*}(X ; \mathbf{Q}) \rightarrow H_{G}^{*}\left(U_{\beta} ; \mathbf{Q}\right)$ are all surjective, and hence the Thom-Gysin maps $H_{G}^{j-2 \lambda(\beta)}\left(S_{\beta} ; \mathbf{Q}\right) \rightarrow$ $H_{G}^{j}\left(U_{\beta} ; \mathbf{Q}\right)$ can be lifted to maps $H_{G}^{j-2 \lambda(\beta)}\left(S_{\beta} ; \mathbf{Q}\right) \rightarrow H_{G}^{j}(X ; \mathbf{Q})$. 
Lemma 7. The direct sum

$$
\bigoplus_{\beta \neq 0} H_{G}^{j-2 \lambda(\beta)}\left(S_{\beta} ; \mathbf{Q}\right) \rightarrow H_{G}^{j}(X ; \mathbf{Q})
$$

of any choice of lifts $H_{G}^{j-2 \lambda(\beta)}\left(S_{\beta} ; \mathbf{Q}\right) \rightarrow H_{G}^{j}(X ; \mathbf{Q})$ of the Thom-Gysin maps $H_{G}^{j-2 \lambda(\beta)}\left(S_{\beta} ; \mathbf{Q}\right) \rightarrow H_{G}^{j}\left(U_{\beta} ; \mathbf{Q}\right)$ is an isomorphism onto its image, which is the kernel of the restriction map $H_{G}^{*}(X ; \mathbf{Q}) \rightarrow H_{G}^{*}\left(X^{\text {ss }} ; \mathbf{Q}\right)$.

Proof. The proof is immediate.

Proposition 8. The restriction map $H_{G}^{*}(X ; \mathbf{Q}) \rightarrow H_{T}^{*}\left(X_{T} ; \mathbf{Q}\right)$ is injective. Its image is contained in the invariant part $\left[H_{T}^{*}\left(X_{T} ; \mathbf{Q}\right)\right]^{W}$ of $H_{T}^{*}\left(X_{T} ; \mathbf{Q}\right)$ under the action of the Weyl group $W$ of $K$.

Proof. Choose an element $\gamma$ of the Lie algebra $\mathbf{t}$ of $T$ such that $\exp \mathbf{R} \gamma$ is dense in $T$. Consider the function $\mu_{\gamma}: X \rightarrow \mathbf{R}$ defined by

$$
\mu_{\gamma}(x)=\mu(x) \cdot \gamma
$$

where $\mu$ is the momentum map defined above. Then $\mu_{\gamma}$ is a nondegenerate Morse function in the sense of Bott [3], and the set of its critical points is $X^{T}$ (see [1] or [8, 3.9]). Let $\left\{F_{i} \mid 1 \leq i \leq s\right\}$ be the connected components of $X^{T}$. Then there is a stratification $\left\{\Sigma_{i} \mid 1 \leq i \leq s\right\}$ of $X$ such that a point $x \in X$ lies in the stratum $\Sigma_{i}$ if its path of steepest descent under $\mu_{\gamma}$ with respect to the Fubini-Study metric has a limit point in $F_{i}$. Each $\Sigma_{i}$ is a locally closed submanifold of $X$ that retracts onto $F_{i}$ (see [3] or [8, 4.6]). We may assume that the indices are chosen in such a way that $\bar{\Sigma}_{i} \subseteq \bigcup_{j \geq i} \Sigma_{j}$ for each $i$, so that $U_{i}=\bigcup_{j \leq i} \Sigma_{j}$ is open in $X$ and contains $\Sigma_{i}$ as a closed submanifold. Since $U_{i}-\Sigma_{i}=U_{i-1}$, there is a long exact sequence (the Thom-Gysin sequence)

$$
\cdots \rightarrow H_{T}^{j-2 \ell(i)}\left(\Sigma_{i} ; \mathbf{Q}\right) \rightarrow H_{T}^{j}\left(U_{i} ; \mathbf{Q}\right) \rightarrow H_{T}^{j}\left(U_{i-1} ; \mathbf{Q}\right) \rightarrow \cdots
$$

where $\ell(i)$ is the complex codimension of $\Sigma_{i}$ in $X$. The composition of the Thom-Gysin map

$$
H_{T}^{j-2 \ell(i)}\left(\Sigma_{i} ; \mathbf{Q}\right) \rightarrow H_{T}^{j}\left(U_{i} ; \mathbf{Q}\right)
$$

with the restriction map

$$
H_{T}^{j}\left(U_{i} ; \mathbf{Q}\right) \rightarrow H_{T}^{j}\left(\Sigma_{i} ; \mathbf{Q}\right)
$$

is given by multiplication by the equivariant Euler class of the normal bundle to $\Sigma_{i}$ in $X$ (cf. [2, p. 535]). Under the isomorphism

$$
H_{T}^{j}\left(\Sigma_{i} ; \mathbf{Q}\right) \rightarrow H_{T}^{j}\left(F_{i} ; \mathbf{Q}\right)
$$

induced by the retraction, this Euler class is identified with the equivariant Euler class of the normal bundle to $\Sigma_{i}$ restricted to $F_{i}$. Since $F_{i}$ is a connected component of the fixed point set of $T$, application of the criterion of Atiyah and 
Bott $([2,13.4])$ shows that this Euler class is not a zero-divisor in $H_{T}^{*}\left(F_{i} ; \mathbf{Q}\right)$. (Note that this means that the Thom-Gysin map

$$
H_{T}^{j-2 \ell(i)}\left(\Sigma_{i} ; \mathbf{Q}\right) \rightarrow H_{T}^{j}\left(U_{i} ; \mathbf{Q}\right)
$$

is injective, so that the Thom-Gysin sequences break up into short exact sequences, i.e., the stratification is $T$-equivariantly perfect.)

Now suppose that $\eta \in H_{T}^{j}(X ; \mathbf{Q})$ is such that its image in $H_{T}^{*}\left(X^{T} ; \mathbf{Q}\right)$ under the restriction map is zero. Since $\Sigma_{i}$ retracts onto $F_{i}$ this means that the image of $\eta$ in $H_{T}^{*}\left(\Sigma_{i} ; \mathbf{Q}\right)$ is zero for each $i$. In particular, the restriction of $\eta$ to $U_{1}=\Sigma_{1}$ is zero. Let $i_{0}$ be the maximal value of $i$ such that the restriction of $\eta$ to $U_{i}$ is zero. We wish to show that $i_{0}=s$. If $i_{0}<s$ let $\zeta \in H^{j}\left(U_{i_{0}+1} ; \mathbf{Q}\right)$ be the image of $\eta$ under restriction and consider the exact sequence

$$
\cdots \rightarrow H_{T}^{j-2 \ell\left(i_{0}+1\right)}\left(\Sigma_{i_{0}+1} ; \mathbf{Q}\right) \stackrel{\sigma}{\rightarrow} H_{T}^{j}\left(U_{i_{0}+1} ; \mathbf{Q}\right) \stackrel{\tau}{\rightarrow} H_{T}^{j}\left(U_{i_{0}} ; \mathbf{Q}\right) \rightarrow \cdots .
$$

By the choice of $i_{0}$ we have $\tau(\zeta)=0$, so

$$
\zeta=\sigma(\xi) \text { for some } \xi \in H_{T}^{j-2 \ell\left(i_{0}+1\right)}\left(\Sigma_{i_{0}+1}\right) .
$$

Therefore if $\psi: H_{T}^{j}\left(U_{i_{0}+1}\right) \rightarrow H_{T}^{j}\left(\Sigma_{i_{0}+1}\right)$ is the restriction map then $\psi(\sigma(\xi))=$ $\psi(\zeta)=0$ by the assumption on $\eta$. But we have just seen that the composition $\psi \circ \sigma$ is injective, so $\xi=0$ and hence $\zeta=0$. This is impossible by the choice of $i_{0}$. Thus $i_{0}=s$ so $U_{i_{0}}=X$ and $\eta=0$.

This proves that the restriction map

$$
H_{T}^{*}(X ; \mathbf{Q}) \rightarrow H_{T}^{*}\left(X^{T} ; \mathbf{Q}\right)
$$

is injective. Since this map respects the action of the Weyl group $W$ and $H_{G}^{*}(X ; \mathbf{Q})$ is naturally isomorphic to $\left[H_{T}^{*}(X ; \mathbf{Q})\right]^{W}$, it restricts to an injection

$$
H_{G}^{*}(X ; \mathbf{Q}) \rightarrow\left[H_{T}^{*}\left(X^{T} ; \mathbf{Q}\right)\right]^{W},
$$

so the result is proved.

Remark 7. The proof of the last proposition shows that the component $\mu_{\gamma}$ : $X \rightarrow \mathbf{R}$ of the momentum map $\mu$ in the direction of $\gamma \in \mathbf{t}$ is a $T_{\gamma}$-equivariantly perfect Morse function, where $T_{\gamma}$ is the subtorus of $T$ generated by $\gamma$, i.e., the closure in $T$ of $\exp \mathbf{R} \gamma$. The critical points of this function are the fixed points of the action of $T_{\gamma}$. Since

$$
H_{T_{\gamma}}^{*}(X ; \mathbf{Q}) \cong H^{*}(X ; \mathbf{Q}) \otimes H^{*}\left(B T_{\gamma} ; \mathbf{Q}\right)
$$

and

$$
H_{T_{\gamma}}^{*}(F ; \mathbf{Q}) \cong H^{*}(F ; \mathbf{Q}) \otimes H^{*}\left(B T_{\gamma} ; \mathbf{Q}\right)
$$

for every connected component $F$ of the set of fixed points of the action of $T_{\gamma}$, it follows that $\mu_{\gamma}$ is a perfect Morse function as well as an equivariantly perfect one. 
Remark 8. The composition $\mu_{T}: X \rightarrow \mathbf{t}^{*}$ of the momentum map $\mu$ with the dual of the inclusion $\mathbf{t} \rightarrow \mathbf{k}$ is a momentum map for the action of $T$ on $K$. The image of any connected component $F$ of the fixed point set $X^{T}$ under $\mu_{T}$ is a single point of $\mathbf{t}^{*}$; in fact, it is a weight, $\alpha(F)$ say, of the representation of $T$ that defines its action on $X$ (see [8,3.5]). Since $\mu_{T}$ is a $T$-invariant map and $T$ acts trivially on $X^{T}$, it is easy to check that there are natural ring isomorphisms

$$
\begin{aligned}
{\left[H_{T}^{*}\left(X^{T} ; \mathbf{Q}\right)\right]^{W} } & \cong\left[\bigoplus_{F \in \mathscr{F}} H^{*}(F ; \mathbf{Q}) \otimes H^{*}(B T ; \mathbf{Q})\right]^{W} \\
& \cong \bigoplus_{F \in \mathscr{F}_{+}} H^{*}(F ; \mathbf{Q}) \otimes H^{*}(B \operatorname{Stab} \alpha(F) ; \mathbf{Q}) \\
& \cong \bigoplus_{F \in \mathscr{F}_{+}} H_{\mathrm{Stab}(\alpha(F)}^{*}(F ; \mathbf{Q})
\end{aligned}
$$

where $\mathscr{F}$ is the set of connected components of $X^{T}$ and $\mathscr{F}_{+}$consists of those $F \in \mathscr{F}$ such that $\alpha(F)$ belongs to the positive Weyl chamber $\mathbf{t}_{+}^{*}$.

It follows from the results just proved in this section that to describe the kernel of the restriction map $H_{G}^{*}(X ; \mathbf{Q}) \rightarrow H_{G}^{*}\left(X^{\mathrm{ss}} ; \mathbf{Q}\right)$ it is enough to choose lifts $H_{G}^{j-2 \lambda(\beta)}\left(S_{\beta} ; \mathbf{Q}\right) \rightarrow H_{G}^{j}(X ; \mathbf{Q})$ of the Thom-Gysin maps $H_{G}^{j-2 \lambda(\beta)}\left(S_{\beta} ; \mathbf{Q}\right) \rightarrow$ $H_{G}^{j}\left(U_{\beta} ; \mathbf{Q}\right)$ and determine their compositions with the restriction maps

$$
H_{G}^{*}(X ; \mathbf{Q}) \rightarrow H_{\mathrm{Stab}(\alpha(F)}^{*}(F ; \mathbf{Q})
$$

for each $F \in \mathscr{F}_{+}$.

Proposition 9. Let $F$ be a connected component of the fixed point set $X^{T}$ of $T$ in $X$ and let $\alpha(F)$ be its image under $\mu_{T}$. Let $\beta$ be an element of the indexing set of the stratification $\left\{S_{\beta} \mid \beta \in \mathscr{B}\right\}$ of $X$. If there is an element $w$ of the Weyl group $W$ such that $w(\alpha(F)) . \beta \geq\|\beta\|^{2}$ then $F \subseteq \overline{S_{\beta}}$; otherwise $F \cap \overline{S_{\beta}}=\emptyset$.

Proof. If there is $w \in W$ such that $w(\alpha(F)) . \beta \geq\|\beta\|^{2}$ then $w(F) \subseteq \overline{Y_{\beta}} \subseteq$ $\overline{S_{\beta}}$ so $F \subseteq \overline{S_{\beta}}$. Otherwise every element of the set $\{w(\alpha(F) \mid w \in W\}$ lies in the open half-plane $\left\{\xi \in \mathbf{t} \mid \xi . \beta<\|\beta\|^{2}\right\}$ and hence so does its convex hull. By [1] this convex hull is the projection onto $\mathbf{t}$ of the adjoint orbit $K \alpha(F)$ of $\alpha(F)$ in $\mathbf{k}$, so if $x \in F$ and $k \in K$ then

$$
\mu(k x) \cdot \beta=k \mu(x) \cdot \beta=k(\alpha(F)) \cdot \beta<\|\beta\|^{2},
$$

which means that $k x \notin \overline{Y_{\beta}}$. Therefore $F \cap K \overline{Y_{\beta}}=\emptyset$. But $\overline{Y_{\beta}}$ is invariant under the parabolic subgroup $P_{\beta}$, which the product of $\operatorname{Stab} \beta$ and the Borel subgroup $B$ of $G$ associated to $\mathbf{t}_{+}$. Since $G=K B$, it follows that $S_{\beta} \subseteq G \overline{Y_{\beta}}=K \overline{Y_{\beta}}$, 
which is closed, so $\overline{S_{\beta}} \subseteq K \overline{Y_{\beta}}$. Thus

$$
F \cap \overline{S_{\beta}}=\emptyset \text {. }
$$

Proposition 10. If $\beta \in \mathscr{B}$ and $F$ is a connected component of $X^{T}$ such that $F \cap \overline{S_{\beta}}=\emptyset$ then the composition of any lift $H_{G}^{j-2 \lambda(\beta)}\left(S_{\beta} ; \mathbf{Q}\right) \rightarrow H_{G}^{j}(X ; \mathbf{Q})$ of the Thom-Gysin map $H_{G}^{j-2 \lambda(\beta)}\left(S_{\beta} ; \mathbf{Q}\right) \rightarrow H_{G}^{j}\left(U_{\beta} ; \mathbf{Q}\right)$ with the restriction map

$$
H_{G}^{*}(X ; \mathbf{Q}) \rightarrow H_{\mathrm{Stab}(\alpha(F)}^{*}(F ; \mathbf{Q})
$$

is zero.

Proof. Since $F \subseteq U_{\beta}-S_{\beta}$ and $\operatorname{Stab} \alpha(F) \subseteq G$, the composition factors through the composition of two maps in the Thom-Gysin sequence. It is therefore zero because this sequence is exact.

Proposition 11. If $\beta \in \mathscr{B}$ and $F$ is a connected component of $X^{T}$ such that $F \subseteq \overline{S_{\beta}}$ then the image of the canonical generator 1 of $H_{G}^{0}\left(S_{\beta} ; \mathbf{Q}\right)$ under the composition of a suitable lift $H_{G}^{0}\left(S_{\beta} ; \mathbf{Q}\right) \rightarrow H_{G}^{2 \lambda(\beta)}(X ; \mathbf{Q})$ of the Thom-Gysin map $H_{G}^{0}\left(S_{\beta} ; \mathbf{Q}\right) \rightarrow H_{G}^{2 \lambda(\beta)}\left(U_{\beta} ; \mathbf{Q}\right)$ with the restriction map

$$
H_{G}^{*}(X ; \mathbf{Q}) \rightarrow H_{\operatorname{Stab}(\alpha(F)}^{*}(F ; \mathbf{Q})
$$

is the restriction $\left.\left[\overline{S_{\beta}}\right]\right|_{F}$ of the equivariant cocycle in $H_{G}^{2 \lambda(\beta)}(X ; \mathbf{Q})$ defined by $\overline{S_{\beta}}$ via Poincaré duality. If, moreover, $F$ does not contain any singular points of $\overline{S_{\beta}}$ (in particular, if $F \subseteq S_{\beta}$ ) then $\left.\left[\overline{S_{\beta}}\right]\right|_{F}$ is the equivariant Euler class of the restriction to $F$ of the normal bundle to $\overline{S_{\beta}}$.

Proof. Note that if we modify the partial order on the indexing set $\mathscr{B}$ so that the codimension $\lambda(\gamma)$ of $S_{\gamma}$ is strictly greater than the codimension $\lambda(\beta)$ of $S_{\beta}$ whenever $\gamma>\beta$ then the restriction map $H_{G}^{j}(X ; \mathbf{Q}) \rightarrow H_{G}^{j}\left(U_{\beta} ; \mathbf{Q}\right)$ is an isomorphism when $j \leq 2 \lambda(\beta)$, so there is no choice in the lift $H_{G}^{0}\left(S_{\beta} ; \mathbf{Q}\right) \rightarrow$ $H_{G}^{2 \lambda(\beta)}(X ; \mathbf{Q})$ of the Thom-Gysin map $H_{G}^{0}\left(S_{\beta} ; \mathbf{Q}\right) \rightarrow H_{G}^{2 \lambda(\beta)}\left(U_{\beta} ; \mathbf{Q}\right)$. The result now follows by chasing through the definition of the Thom-Gysin map.

Corollary 2. If $\beta \in \mathscr{B}$ and $F$ is a connected component of $X^{T}$ such that $F \subseteq S_{\beta}$ then there is a lift

$$
H_{G}^{*}\left(S_{\beta} ; \mathbf{Q}\right) \rightarrow H_{G}^{*+2 \lambda(\beta)}(X ; \mathbf{Q})
$$

of the Thom-Gysin map $H_{G}^{*}\left(S_{\beta} ; \mathbf{Q}\right) \rightarrow H_{G}^{*+2 \lambda(\beta)}\left(U_{\beta} ; \mathbf{Q}\right)$ whose composition with the restriction map

$$
H_{G}^{*}(X ; \mathbf{Q}) \rightarrow H_{\mathrm{Stab}(\alpha(F)}^{*}(F ; \mathbf{Q})
$$

sends any $\xi \in H_{G}^{*}\left(S_{\beta} ; \mathbf{Q}\right)$ to the product of $\left.\xi\right|_{F}$ with the equivariant Euler class of the restriction to $F$ of the normal bundle to $S_{\beta}$ in $X$. 
Proof. This follows by chasing through the definition of the Thom-Gysin map.

Corollary 3. If $\beta \in \mathscr{B}$ and $F$ is a connected component of $X^{T}$ such that $F \subseteq \overline{S_{\beta}}$ and the restriction map $H_{G}^{*}(X ; \mathbf{Q}) \rightarrow H_{G}^{*}\left(S_{\beta} ; \mathbf{Q}\right)$ is surjective, then given any right inverse

$$
\rho: H_{G}^{*}\left(S_{\beta} ; \mathbf{Q}\right) \rightarrow H_{G}^{*}(X ; \mathbf{Q})
$$

of this map there is a lift

$$
H_{G}^{*}\left(S_{\beta} ; \mathbf{Q}\right) \rightarrow H_{G}^{*+2 \lambda(\beta)}(X ; \mathbf{Q})
$$

of the Thom-Gysin map $H_{G}^{*}\left(S_{\beta} ; \mathbf{Q}\right) \rightarrow H_{G}^{*+2 \lambda(\beta)}\left(U_{\beta} ; \mathbf{Q}\right)$ whose composition with the restriction map

$$
H_{G}^{*}(X ; \mathbf{Q}) \rightarrow H_{\mathrm{Stab}(\alpha(F)}^{*}(F ; \mathbf{Q})
$$

sends any $\xi \in H_{G}^{*}\left(S_{\beta} ; \mathbf{Q}\right)$ to $\left.\left.\rho(\xi)\right|_{F}\left[\overline{S_{\beta}}\right]\right|_{F}$.

Proof. This follows since the Thom-Gysin map is a homomorphism of $H_{G}^{*}(X ; \mathbf{Q})$-modules.

We have two similar corollaries.

Corollary 4. If $\beta \in \mathscr{B}$ and $F$ is a connected component of $X^{T}$ such that $F \subseteq \overline{S_{\beta}}$ and the restriction map $H_{G}^{*}\left(\overline{S_{\beta}} ; \mathbf{Q}\right) \rightarrow H_{G}^{*}\left(S_{\beta} ; \mathbf{Q}\right)$ is surjective, then given any right inverse

$$
\hat{\rho}: H_{G}^{*}\left(S_{\beta} ; \mathbf{Q}\right) \rightarrow H_{G}^{*}\left(\overline{S_{\beta}} ; \mathbf{Q}\right)
$$

of this map there is a lift

$$
H_{G}^{*}\left(S_{\beta} ; \mathbf{Q}\right) \rightarrow H_{G}^{*+2 \lambda(\beta)}(X ; \mathbf{Q})
$$

of the Thom-Gysin map $H_{G}^{*}\left(S_{\beta} ; \mathbf{Q}\right) \rightarrow H_{G}^{*+2 \lambda(\beta)}\left(U_{\beta} ; \mathbf{Q}\right)$ whose composition with the restriction map

$$
H_{G}^{*}(X ; \mathbf{Q}) \rightarrow H_{\operatorname{Stab}(\alpha(F)}^{*}(F ; \mathbf{Q})
$$

sends any $\xi \in H_{G}^{*}\left(S_{\beta} ; \mathbf{Q}\right)$ to $\left.\left.\hat{\rho}(\xi)\right|_{F}\left[\overline{S_{\beta}}\right]\right|_{F}$.

Corollary 5. If $\beta \in \mathscr{B}$ and $w \in W$ and $F$ is a connected component of $X^{T}$ such that $w(F) \subseteq \overline{Y_{\beta}}$ and the restriction map

$$
H_{\mathrm{Stab} \beta}^{*}\left(\overline{Y_{\beta}} ; \mathbf{Q}\right) \rightarrow H_{\mathrm{Stab} \beta}^{*}\left(Z_{\beta}^{\mathrm{ss}} ; \mathbf{Q}\right)
$$

is surjective, then given any right inverse

$$
\tilde{\rho}: H_{\mathrm{Stab} \beta}^{*}\left(Z_{\beta}^{\mathrm{ss}} ; \mathbf{Q}\right) \rightarrow H_{\mathrm{Stab} \beta}^{*}\left(\overline{Y_{\beta}} ; \mathbf{Q}\right)
$$

of this map there is a lift

$$
H_{G}^{*}\left(S_{\beta} ; \mathbf{Q}\right) \rightarrow H_{G}^{*+2 \lambda(\beta)}(X ; \mathbf{Q})
$$


of the Thom-Gysin map $H_{G}^{*}\left(S_{\beta} ; \mathbf{Q}\right) \rightarrow H_{G}^{*+2 \lambda(\beta)}\left(U_{\beta} ; \mathbf{Q}\right)$ whose composition with the restriction map

$$
H_{G}^{*}(X ; \mathbf{Q}) \rightarrow H_{\mathrm{Stab}(\alpha(F)}^{*}(F ; \mathbf{Q})
$$

sends any $\xi \in H_{\mathrm{Stab} \beta}^{*}\left(Z_{\beta}^{\mathrm{ss}} ; \mathbf{Q}\right) \cong H_{G}^{*}\left(S_{\beta} ; \mathbf{Q}\right)$ to $\left.\left.w \tilde{\rho}(\xi)\right|_{F}\left[\overline{S_{\beta}}\right]\right|_{F}$.

These corollaries can be made to apply more generally than the preceding one because of the following lemma.

Lemma 8. (i) If $\overline{S_{\beta}}$ is nonsingular then the restriction map

$$
H_{G}^{*}\left(\overline{S_{\beta}} ; \mathbf{Q}\right) \rightarrow H_{G}^{*}\left(S_{\beta} ; \mathbf{Q}\right)
$$

is surjective.

(ii) If $\overline{Y_{\beta}}$ is nonsingular then the restriction map

$$
H_{\mathrm{Stab} \beta}^{*}\left(\overline{Y_{\beta}} ; \mathbf{Q}\right) \rightarrow H_{\mathrm{Stab} \beta}^{*}\left(Z_{\beta}^{\mathrm{ss}} ; \mathbf{Q}\right)
$$

is surjective.

Proof. (i) The stratification of $\overline{S_{\beta}}$ defined by the function $\|\mu\|^{2}$ is equivariantly perfect over $\mathbf{Q}$ (by property (iv) of the stratification applied to the action of $G$ on $\overline{S_{\beta}}$ ) and it has $S_{\beta}$ as an open stratum. Therefore the restriction map

$$
H_{G}^{*}\left(\overline{S_{\beta}} ; \mathbf{Q}\right) \rightarrow H_{G}^{*}\left(S_{\beta} ; \mathbf{Q}\right)
$$

is surjective.

(ii) The restriction map

$$
H_{\mathrm{Stab} \beta}^{*}\left(Z_{\beta} ; \mathbf{Q}\right) \rightarrow H_{\mathrm{Stab} \beta}^{*}\left(Z_{\beta}^{\mathrm{ss}} ; \mathbf{Q}\right)
$$

is surjective by property (iv) of the stratification applied to the action of $\operatorname{Stab} \beta$ on the nonsingular projective subvariety $Z_{\beta}$ of $X$.

The restriction to $\overline{Y_{\beta}}$ of the momentum map $\mu_{T}: X \rightarrow \mathbf{t}^{*}$ is a momentum map for the action of $T$ on $\overline{Y_{\beta}}$. By the first remark of this section its component $\mu_{\beta}$ in the direction of $\beta$ is a perfect Morse function (over the rationals) on $\overline{Y_{\beta}}$, and hence the restriction map from the cohomology of $\overline{Y_{\beta}}$ to the cohomology of the subset where $\mu_{\beta}$ attains its minimum value is surjective. Since $\mu_{\beta}(x) \geq\|\beta\|^{2}$ for all $x \in \overline{Y_{\beta}}$ and, moreover, $\mu_{\beta}(x)=\|\beta\|^{2}$ if and only if $x \in Z_{\beta}$, this function attains its minimum value precisely on $Z_{\beta}$. Since the $\operatorname{Stab} \beta$-equivariant cohomology of $Z_{\beta}$ and $\overline{Y_{\beta}}$ is the tensor product of ordinary cohomology with the cohomology of the classifying space $B \operatorname{Stab} \beta$ the result follows.

If $\overline{Y_{\beta}}$ or $\overline{S_{\beta}}$ is nonsingular for each $\beta \in \mathscr{B}$ then we can describe the kernel of the restriction map $H_{G}^{*}(X ; \mathbf{Q}) \rightarrow H_{G}^{*}\left(X^{\text {ss }} ; \mathbf{Q}\right)$ by using Corollaries 4 and 5 to choose lifts $H_{G}^{j-2 \lambda(\beta)}\left(S_{\beta} ; \mathbf{Q}\right) \rightarrow H_{G}^{j}(X ; \mathbf{Q})$ of the Thom-Gysin maps $H_{G}^{j-2 \lambda(\beta)}\left(S_{\beta} ; \mathbf{Q}\right) \rightarrow H_{G}^{j}\left(U_{\beta} ; \mathbf{Q}\right)$ and to determine their compositions with the 
restriction maps

$$
H_{G}^{*}(X ; \mathbf{Q}) \rightarrow H_{\mathrm{Stab}(\alpha(F)}^{*}(F ; \mathbf{Q})
$$

for each $F \in \mathscr{F}_{+}$.

It is not in general the case that $\overline{Y_{\beta}}$ or $\overline{S_{\beta}}$ is nonsingular for each $\beta \in \mathscr{B}$. Nonetheless, for any $\beta \in \mathscr{B}$ we can resolve the singularities of $\overline{S_{\beta}}$ in $X$ by a succession of blow-ups along nonsingular $G$-invariant subvarieties ${ }^{7}$, giving a projective variety with an action of $G$ which can be appropriately linearised (cf. [10]). So let us consider the following situation.

Let $C$ be a nonsingular $G$-invariant projective subvariety of $X$ contained in the union of those strata $S_{\gamma}$ with $\gamma>\beta$, and let $\pi: \widetilde{X} \rightarrow X$ be the result of blowing up $X$ along $C$. Let $E$ be the exceptional divisor and let $\widetilde{S}_{\beta}$ and $\tilde{Y}_{\beta}$ be the proper transforms of $\overline{S_{\beta}}$ and $\overline{Y_{\beta}}$ in $\tilde{X}$. The images of the natural injection

$$
\pi^{*}: H_{G}^{*}(X ; \mathbf{Q}) \rightarrow H_{G}^{*}(\tilde{X} ; \mathbf{Q})
$$

and the Thom-Gysin map associated to $E$

$$
\tau_{E}: H_{G}^{*-2}(E ; \mathbf{Q}) \rightarrow H_{G}^{*}(\tilde{X} ; \mathbf{Q})
$$

span $H_{G}^{*}(\widetilde{X} ; \mathbf{Q})$, and their intersection is the image under $\pi^{*}$ of the image of the Thom-Gysin map

$$
\tau_{C}: H_{G}^{*-2 c}(C ; \mathbf{Q}) \rightarrow H_{G}^{*}(X ; \mathbf{Q})
$$

where $c$ is the complex codimension of $C$ in $X$. The image of this ThomGysin map is of course equal to the kernel of the restriction map

$$
H_{G}^{*}(X ; \mathbf{Q}) \rightarrow H_{G}^{*}(X-C ; \mathbf{Q}),
$$

which is contained in the kernel of the restriction map

$$
H_{G}^{*}(X ; \mathbf{Q}) \rightarrow H_{G}^{*}\left(X-\bigcup_{\gamma>\beta} S_{\gamma} ; \mathbf{Q}\right)
$$

Choose any complement to the image of $H_{G}^{*-2 c}(C ; \mathbf{Q})$ in $H_{G}^{*-2}(E ; \mathbf{Q})$ and thus decompose $H_{G}^{*}(\tilde{X} ; \mathbf{Q})$ as

$$
\pi^{*} H_{G}^{*}(X ; \mathbf{Q}) \oplus \tau_{E}\left(H_{G}^{*-2}(E ; \mathbf{Q}) / H_{G}^{*-2 c}(C ; \mathbf{Q})\right),
$$

where

$$
H_{G}^{*}(E ; \mathbf{Q}) \cong H_{G}^{*}(C ; \mathbf{Q}) \otimes H^{*}\left(\mathbf{P}_{c-1} ; \mathbf{Q}\right)
$$

Note that in dimensions less than $2 c$ the image of $H_{G}^{*-2 c}(C ; \mathbf{Q})$ in $H_{G}^{*}(X ; \mathbf{Q})$ is zero, so this decomposition does not depend on any choice. In particular,

\footnotetext{
${ }^{7}$ This depends on Hironaka's equivariant resolution of singularities theorem, which was announced in [7] 9, Remark 8, but which does not seem to have a complete published proof.
} 
this is true in dimension $2 \operatorname{dim} S_{\beta}$. Let

$$
\theta: H_{G}^{*}(\widetilde{X} ; \mathbf{Q}) \rightarrow H_{G}^{*}(X ; \mathbf{Q})
$$

be the projection with respect to this decomposition. Then if

$$
H_{G}^{j-2 \lambda(\beta)}\left(S_{\beta} ; \mathbf{Q}\right) \rightarrow H_{G}^{j}(\widetilde{X} ; \mathbf{Q})
$$

is a lift of the Thom-Gysin map $H_{G}^{j-2 \lambda(\beta)}\left(S_{\beta} ; \mathbf{Q}\right) \rightarrow H_{G}^{j}\left(U_{\beta} ; \mathbf{Q}\right)$, its composition with the projection $\theta$ is a lift to $H_{G}^{j}(X ; \mathbf{Q})$ of the Thom-Gysin map $H_{G}^{j-2 \lambda(\beta)}\left(S_{\beta} ; \mathbf{Q}\right) \rightarrow H_{G}^{j}\left(U_{\beta} ; \mathbf{Q}\right)$. In particular, $\theta\left[\widetilde{S}_{\beta}\right]=\left[\overline{S_{\beta}}\right]$.

If $\widetilde{F}$ is a connected component of the fixed point set $\widetilde{X}^{T}$ then its image $\pi(\widetilde{F})$ in $X$ is contained in a connected component of $X^{T}$. If $F$ does not meet the centre $C$ of the blow-up, then $\pi: \widetilde{F} \rightarrow F$ is an isomorphism. If $F \subseteq C$ then $\pi: \widetilde{F} \rightarrow F$ is a fibration with a projective space, $\mathbf{P}_{k}$ say, as fibre, and

$$
H_{T}^{*}(\widetilde{F} ; \mathbf{Q}) \cong H_{T}^{*}(F ; \mathbf{Q}) \otimes H^{*}\left(\mathbf{P}_{k} ; \mathbf{Q}\right) .
$$

Finally if $F$ meets $C$ but is not contained in it then either $\widetilde{F}$ is the blowup of $F$ along $F \cap C$, which is nonsingular since it is a union of connected components of $C^{T}$, or $\pi: \widetilde{F} \rightarrow F \cap C$ is a fibration as above with a projective space, $\mathbf{P}_{k}$ say, as fibre, and

$$
H_{T}^{*}(\widetilde{F} ; \mathbf{Q}) \cong H_{T}^{*}(F \cap C ; \mathbf{Q}) \otimes H^{*}\left(\mathbf{P}_{k} ; \mathbf{Q}\right) .
$$

Using this one obtains an explicit description of the embedding

$$
\pi^{*}: H_{T}^{*}\left(X^{T} ; \mathbf{Q}\right) \rightarrow H_{T}^{*}\left(\widetilde{X}^{T} ; \mathbf{Q}\right) .
$$

One can then extend the projection $\theta: H_{G}^{*}(\tilde{X} ; \mathbf{Q}) \rightarrow H_{G}^{*}(X ; \mathbf{Q})$ to a map $\theta^{T}: H_{T}^{*}\left(\widetilde{X}^{T} ; \mathbf{Q}\right) \rightarrow H_{T}^{*}\left(X^{T} ; \mathbf{Q}\right)$. Then the composition of the inclusion

$$
H_{G}^{*}(X ; \mathbf{Q}) \rightarrow H_{T}^{*}\left(X^{T} ; \mathbf{Q}\right) \cong \bigoplus_{F} H_{\mathrm{Stab}(\alpha(F)}^{*}(F ; \mathbf{Q})
$$

with the lift to $H_{G}^{j}(X ; \mathbf{Q})$ chosen above of the Thom-Gysin map

$$
H_{G}^{j-2 \lambda(\beta)}\left(S_{\beta} ; \mathbf{Q}\right) \rightarrow H_{G}^{j}\left(U_{\beta} ; \mathbf{Q}\right)
$$

is the composition of $\theta^{T}$ with the inclusion

$$
H_{G}^{*}(\widetilde{X} ; \mathbf{Q}) \rightarrow H_{T}^{*}\left(\tilde{X}^{T} ; \mathbf{Q}\right) \cong \bigoplus_{\tilde{F}} H_{\mathrm{Stab}(\alpha(\widetilde{F})}^{*}(\widetilde{F} ; \mathbf{Q})
$$

and the lift to $H_{G}^{j}(\tilde{X} ; \mathbf{Q})$ of the Thom-Gysin map $H_{G}^{j-2 \lambda(\beta)}\left(S_{\beta} ; \mathbf{Q}\right) \rightarrow$ $H_{G}^{j}\left(U_{\beta} ; \mathbf{Q}\right)$.

In this way it is possible to determine the kernel of the restriction map $H_{G}^{*}(X ; \mathbf{Q}) \rightarrow H_{G}^{*}\left(X^{\text {ss }} ; \mathbf{Q}\right)$ using Corollary 4 and Lemma 8 applied to suitable blow-ups of $X$. 
Examples. (i) Let $G=\operatorname{SL}(2 ; \mathbf{C})$ act on $X=\mathbf{P}_{n}$ via the $n$th symmetric power $G \rightarrow \mathrm{GL}(n+1 ; \mathrm{C})$ of the standard representation of $G$ on $\mathbf{C}^{2}$. Equivalently we can identify $\mathbf{P}_{n}$ with the space of unordered $n$-tuples of points in $\mathbf{P}_{1}=$ $\mathbf{C} \cup\{\infty\}$ acted on by Möbius transformations. The semistable $n$-tuples are those containing no point of $\mathbf{P}_{1}$ strictly more than $n / 2$ times; the stable ones are those containing no point of $\mathbf{P}_{1}$ at least $n / 2$ times (cf. $\left.[8, \S 16]\right)$. We shall assume that $n$ is odd, so that $X^{\text {ss }}=X^{\text {s }}$.

Let $T$ be the torus consisting of diagonal $2 \times 2$ matrices with entries $e^{i \theta}$, $e^{-i \theta}$. Then the fixed point set $X^{T}$ is $\left\{q_{0}, \ldots, q_{n}\right\}$ where $q_{\ell}$ is the unordered $n$-tuple consisting of points $\ell$ of which are $\infty$ and the rest 0 . Hence

$$
H_{T}^{*}\left(X^{T} ; \mathbf{Q}\right) \cong \bigoplus_{\ell=0}^{n} H^{*}(B T ; \mathbf{Q})
$$

where $H^{*}(B T ; \mathbf{Q})=\mathbf{Q}[\alpha]$ is a polynomial algebra over $\mathbf{Q}$ with one generator $\alpha$ of degree 2 . The Weyl group $W$ is cyclic of order 2 and its generator sends $\alpha$ to $-\alpha$, so

$$
H^{*}(B G ; \mathbf{Q})=\left[H^{*}(B T ; \mathbf{Q})\right]^{W}=\mathbf{Q}\left[\alpha^{2}\right] .
$$

$H^{*}\left(\mathbf{P}_{n} ; \mathbf{Q}\right)$ has one generator, $\xi$ say, of degree two, and we can regard $\alpha$ and $\xi$ as generators of $H_{G}^{*}\left(\mathbf{P}_{n} ; \mathbf{Q}\right)$, subject to the relation

$$
\prod_{\ell=0}^{n}\left(\xi-\alpha_{\ell}\right)=0
$$

where $\alpha_{0}, \ldots, \alpha_{n}$ are the weights of the representation $G \rightarrow \mathrm{GL}(n+1 ; \mathbf{C})$, identified in the usual way with elements of $H^{*}(B T ; \mathbf{Q})$. These weights are

$$
n \alpha, \ldots,(n-2 \ell) \alpha, \ldots,-n \alpha,
$$

so the relation is

$$
\prod_{\ell=0}^{[n / 2]}\left(\xi^{2}-(n-2 \ell)^{2} \alpha^{2}\right)=0 .
$$

The restriction map $H^{*}\left(\mathbf{P}_{n} ; \mathbf{Q}\right) \rightarrow H_{T}^{*}\left(X^{T} ; \mathbf{Q}\right)$ is given by

$$
\begin{gathered}
\alpha^{2} \mapsto\left(\alpha^{2}, \ldots, \alpha^{2}, \ldots, \alpha^{2}\right), \\
\xi \mapsto(n \alpha, \ldots,(n-2 \ell) \alpha, \ldots,-n \alpha) .
\end{gathered}
$$

The stratification $\left\{S_{\beta} \mid \beta \in \mathscr{B}\right\}$ is indexed by the set of weights of the representation $G \rightarrow \mathrm{GL}(n+1$; C) lying in the positive Weyl chamber or, equivalently, by integers $k$ satisfying $[n / 2]<k \leq n$. The stratum $S_{k}$ consists of all $n$ tuples containing some point of $\mathbf{P}_{1}$ precisely $k$ times. Its closure $\overline{S_{k}}$ consists of all $n$-tuples containing some point of $\mathbf{P}_{1}$ at least $k$ times and equals $G \overline{Y_{k}}$ where $\overline{Y_{k}}$ consists of all $n$-tuples containing the point at infinity in $\mathbf{P}_{1}$ at least $k$ times. This is a nonsingular subvariety of $\mathbf{P}_{n}$, which can be identified with $\mathbf{P}_{n-k}$. In fact, $\overline{S_{k}}=G \times{ }_{B} \overline{Y_{k}}$ where $B$ is the Borel subgroup of $G$ consisting of upper triangular matrices. 
The action of $T$ on the normal to $\overline{Y_{k}}$ at any point $q_{\ell}$ belonging to $\overline{Y_{k}}$ (i.e., such that $\ell \geq k$ ) has weights

$$
2 \ell \alpha, 2(\ell-1) \alpha, \ldots, 2(\ell-k+1) \alpha,
$$

so the $T$-equivariant Euler class of the normal bundle to $\overline{Y_{k}}$ restricted to the point $q_{\ell}$ is $\left(2^{k} \ell ! /(\ell-k) !\right) \alpha^{k}$. Since $\overline{S_{k}}=G \times_{B} \overline{Y_{k}}$ and the weight of the adjoint action of $T$ on $G / B$ is $2 \alpha$, it follows (for example from consideration of a resolution of the singularities of $\left.\overline{S_{\beta}}\right)$ that the restriction to $H_{T}^{*}\left(\left\{q_{\ell}\right\} ; \mathbf{Q}\right)$ of the equivariant cohomology class $\left[\overline{S_{\beta}}\right]$ in $H_{G}^{*}(X ; \mathbf{Q})$ is $\left(2^{k-1} \ell ! /(\ell-k) !\right) \alpha^{k-1}$. As

$$
H_{G}^{*}\left(S_{k} ; \mathbf{Q}\right) \cong H_{T}^{*}\left(Y_{k} ; \mathbf{Q}\right) \cong H^{*}(B T ; \mathbf{Q}),
$$

the image in $H_{T}^{*}\left(X^{T} ; \mathbf{Q}\right)$ of the kernel of the restriction map $H_{G}^{*}(X ; \mathbf{Q}) \rightarrow$ $H_{G}^{*}\left(X^{\text {ss }} ; \mathbf{Q}\right)$ is $\bigoplus_{[n / 2]<k \leq n} \operatorname{im}\left(\rho_{k}\right)$ where

$$
\rho_{k}: H^{*-2(k-1)}(B T ; \mathbf{Q}) \rightarrow \bigoplus_{0 \leq \ell \leq n} H^{*}(B T ; \mathbf{Q})
$$

has $\ell$ th component zero if $n-k<\ell<k$, multiplication by

$$
\left(2^{k-1} \ell ! /(\ell-k) !\right) \alpha^{k-1} \quad \text { if } \ell \geq k,
$$

and

$$
\left((-2)^{k-1}(n-\ell) ! /(n-\ell-k) !\right) \alpha^{k-1} \text { if } \ell \leq n-k .
$$

Note that a complete set of relations is given by the elements of $H_{G}^{*}(X ; \mathbf{Q})$ mapping to $\left\{\rho_{k}(1), \rho_{k}(\alpha) \mid[n / 2]<k \leq n\right\}$ since $\alpha^{2} \in H_{G}^{*}(X ; \mathbf{Q})$.

From this the kernel of the restriction map can be worked out explicitly. A general element of $H_{G}^{*}(X ; \mathbf{Q})$ can be expressed as a polynomial $p\left(\xi, \alpha^{2}\right)$ in $\xi$ and $\alpha^{2}$ with rational coefficients. This is mapped to the element of $H_{T}^{*}\left(X^{T} ; \mathbf{Q}\right) \cong \bigoplus_{0 \leq \ell \leq n} H^{*}(B T ; \mathbf{Q})$ with $\ell$ th coefficient $p\left((n-2 \ell) \alpha, \alpha^{2}\right)$. Suppose that $n / 2<k \leq n$ and consider the polynomial

$$
r(\xi, \alpha)=\frac{1}{2}\left(\prod_{n-k<i \leq n}(\xi+(n-2 i) \alpha)-\prod_{n-k<i \leq n}(\xi-(n-2 i) \alpha)\right) .
$$

It is homogeneous of degree $k$ in $\xi$ and $\alpha$ and it is odd with respect to $\alpha$, so we can write

$$
r(\xi, \alpha)=\alpha p\left(\xi, \alpha^{2}\right)
$$

for some polynomial $p\left(\xi, \alpha^{2}\right)$ in $\xi$ and $\alpha^{2}$. Since

$$
\prod_{n-k<i \leq n}(\xi-(n-2 i) \alpha)=\prod_{0 \leq i<k}(\xi+(n-2 j) \alpha),
$$

it follows that

$$
p\left((n-2 \ell) \alpha, \alpha^{2}\right)=0
$$


if $n-k<\ell<k$ and that if $\ell \geq k$ then

$p\left((n-2 \ell) \alpha, \alpha^{2}\right)=\frac{1}{2} \alpha^{k-1} \prod_{n-k<i \leq n}((n-2 \ell)-(n-2 i))=\left(2^{k-1} \ell ! /(\ell-k) !\right) \alpha^{k-1}$,

while if $\ell<n-k$ then

$$
p\left((n-2 \ell) \alpha, \alpha^{2}\right)=\left((-2)^{k-1}(n-\ell) ! /(n-\ell-k) !\right) \alpha^{k-1} .
$$

Therefore

$$
\left[\overline{S_{k}}\right]=\left(\prod_{n-k<i \leq n}(\xi+(n-2 i) \alpha)-\prod_{n-k<i \leq n}(\xi-(n-2 i) \alpha)\right) / 2 \alpha
$$

and by a similar argument the general element of the kernel of the restriction $\operatorname{map} H_{G}^{*}(X ; \mathbf{Q}) \rightarrow H_{G}^{*}\left(X^{\mathrm{ss}} ; \mathbf{Q}\right)$ is

$$
\left.q(\xi, \alpha) \prod_{n-k<i \leq n}(\xi+(n-2 i) \alpha)-q(\xi,-\alpha) \prod_{n-k<i \leq n}(\xi-(n-2 i) \alpha)\right) / \alpha
$$

for some $n / 2<k \leq n$ and polynomial $q$ in two variables with rational coefficients.

(ii) A closely related example is given by letting $G=\mathrm{SL}(2 ; \mathrm{C})$ act by Möbius transformations on the space $X=\left(\mathbf{P}_{1}\right)^{n}$ of ordered $n$-tuples of points of $\mathbf{P}_{1}$. Again the semistable $n$-tuples are those containing no point of $\mathbf{P}_{1}$ strictly more than $n / 2$ times; the stable ones are those containing no point of $\mathbf{P}_{1}$ at least $n / 2$ times (cf. $[8, \S 16]$ ). Again we shall assume that $n$ is odd, so that $X^{\text {ss }}=X^{\mathrm{s}}$.

As before let $T$ be the torus consisting of diagonal $2 \times 2$ matrices with entries $e^{i \theta}, e^{-i \theta}$. Then the fixed point set $X^{T}$ is $\left\{q_{R} \mid R \subseteq\{0, \ldots, n\}\right\}$ where the $j$ th component of $q_{R}$ is $\infty$ if $j \in R$ and 0 if $j \notin R$. Hence

$$
H_{T}^{*}\left(X^{T} ; \mathbf{Q}\right) \cong \bigoplus_{R \subseteq\{0, \ldots, n\}} H^{*}(B T ; \mathbf{Q})
$$

$H^{*}\left(\left(\mathbf{P}_{1}\right)^{n} ; \mathbf{Q}\right)$ has $n$ generators, $\xi_{1}, \ldots, \xi_{n}$ say, of degree two, and we can regard $\alpha$ and $\xi_{1}, \ldots, \xi_{n}$ as generators of $H_{G}^{*}\left(\mathbf{P}_{n} ; \mathbf{Q}\right)$, subject to the relations $\xi_{j}^{2}=\alpha^{2}$ for $1 \leq j \leq n$. The $R$ th component of the restriction map $H^{*}\left(\left(\mathbf{P}_{1}\right)^{n} ; \mathbf{Q}\right) \rightarrow H_{T}^{*}\left(X^{T} ; \mathbf{Q}\right)$ maps $\alpha^{2}$ to itself and $\xi_{j}$ to $\alpha$ if $j \in R$ and $-\alpha$ otherwise. The stratification $\left\{S_{\beta} \mid \beta \in \mathscr{B}\right\}$ corresponds exactly to the stratification of $\mathbf{P}_{n}$ described in the previous example under the identification of $\mathbf{P}_{n}$ with the quotient of $\left(\mathbf{P}_{1}\right)^{n}$ by the obvious action of the symmetric group $\Sigma_{n}$. It is indexed by the intersection with the positive Weyl chamber of the set of weights of the $n$th tensor power $G \rightarrow \operatorname{GL}\left(2^{n} ; \mathbf{C}\right)$ of the standard two-dimensional representation of $G$ or, equivalently, by integers $k$ satisfying $[n / 2]<k \leq n$. The connected components of the stratum $S_{k}$ are indexed by the subsets $Q$ of $\{1, \ldots, n\}$ containing precisely $k$ elements. The component $S_{Q}$ consists of all $\left(x_{1}, \ldots, x_{n}\right) \in\left(\mathbf{P}_{1}\right)^{n}$ for which there is some point $p$ of $\mathbf{P}_{1}$ such that $x_{j}=p$ if and only if $j \in Q$. Its closure $\overline{S_{Q}}$ consists of all 
$\left(x_{1}, \ldots, x_{n}\right) \in\left(\mathbf{P}_{1}\right)^{n}$ for which there is some point $p$ of $\mathbf{P}_{1}$ such that $x_{j}=p$ if $j \in Q$; it is nonsingular and can be identified with $\mathbf{P}_{1}^{n-k+1}$. However, the closure of the stratum $S_{k}$ itself is singular; its singularities can be resolved by successively blowing up the proper transforms of the subvarieties $\overline{S_{j}}$ with $j>k$ in decreasing order. The proper transform of $\overline{S_{k}}$ after these blow-ups is a disjoint union of $\left(\begin{array}{l}n \\ k\end{array}\right)$ copies of $\mathbf{P}_{1}^{n-k+1}$.

If $Q$ has $k>n / 2$ elements the action of $T$ on the normal to $\overline{S_{Q}}$ at any point $q_{R}$ belonging to $\overline{S_{Q}}$ (i.e., such that $R \supseteq Q$ or $\{1, \ldots, n\}-R \supseteq Q$ ) has the same weight repeated $k-1$ times, namely, $2 \alpha$ if $R \supseteq Q$ or $-2 \alpha$ if $\{1, \ldots, n\}-R \supseteq Q$. Therefore the $T$-equivariant Euler class of the normal bundle to $\overline{S_{Q}}$ restricted to the point $q_{R}$ is $( \pm 2)^{k-1} \alpha^{k-1}$. As $S_{Q}=G \times{ }_{B} Y_{Q}$ where $B$ is the Borel subgroup of $G$ consisting of upper triangular matrices and $Y_{Q}$ is the contractible subset of $X$ consisting of all $\left(x_{1}, \ldots, x_{n}\right) \in\left(\mathbf{P}_{1}\right)^{n}$ such that $x_{j}=\infty$ if and only if $j \in Q$, we have

$$
H_{G}^{*}\left(S_{Q} ; \mathbf{Q}\right) \cong H_{T}^{*}\left(Y_{Q} ; \mathbf{Q}\right) \cong H^{*}(B T ; \mathbf{Q})
$$

Therefore the image in $H_{T}^{*}\left(X^{T} ; \mathbf{Q}\right)$ of the kernel of the restriction map $H_{G}^{*}(X ; \mathbf{Q}) \rightarrow H_{G}^{*}\left(X^{\mathrm{ss}} ; \mathbf{Q}\right)$ is

$$
\bigoplus_{Q \subseteq\{1, \ldots, n\}, \operatorname{card} Q>[n / 2]} \operatorname{im}\left(\rho_{Q}\right)
$$

where if $Q$ has $k$ elements then

$$
\rho_{Q}: H^{*-2(k-1)}(B T ; \mathbf{Q}) \rightarrow \bigoplus_{R \subseteq\{1, \ldots, n\}} H^{*}(B T ; \mathbf{Q})
$$

has $R$ th component zero unless $R \supseteq Q$ or $\{1, \ldots, n\}-R \supseteq Q$ and multiplication by $( \pm 2)^{k-1} \alpha^{k-1}$ if $R \supseteq Q$ or $\{1, \ldots, n\}-R \supseteq Q$.

As before, from this the kernel of the restriction map

$$
H_{G}^{*}(X ; \mathbf{Q}) \rightarrow H_{G}^{*}\left(X^{\mathrm{ss}} ; \mathbf{Q}\right)
$$

can be worked out explicitly. It consists of all elements of $H_{G}^{*}(X ; \mathbf{Q})$ of the form

$$
\left(q(\xi, \alpha) \prod_{i \in Q}\left(\xi_{i}+\alpha\right)-q(\xi,-\alpha) \prod_{i \in Q}\left(\xi_{i}-\alpha\right)\right) / 2 \alpha
$$

for some $Q \subseteq\{1, \ldots, n\}$ and some polynomial $q$ in two variables with rational coefficients.

The results of the first example can be obtained from this one by noting that the rational cohomology of the quotient of a manifold $Y$ by the action of the symmetric group $\Sigma_{n}$ is the $\Sigma$-invariant part of $H^{*}(Y ; \mathbf{Q})$. 


\section{REFERENCES}

1. M. F. Atiyah, Convexity and commuting Hamiltonians, Bull. London Math. Soc. 14 (1982), $1-15$.

2. M. F. Atiyah and R. Bott, The Yang-Mills equations over Riemann surfaces, Philos. Trans. Roy. Soc. London Ser. A 308 (1982), 523-615.

3. R. Bott, Nondegenerate critical manifolds, Ann. of Math. (2) 60 (1954), 248-261.

4. D. Gieseker, $A$ degeneration of the moduli space of stable bundles, J. Differential Geom. 19 (1984), 173-206.

5. G. Harder and M. S. Narasimhan, On the cohomology groups of moduli spaces of vector bundles over curves, Math. Ann. 212 (1975), 215-248.

6. R. Hartshorne, Algebraic geometry, Graduate Texts in Math., vol. 52, Springer-Verlag, New York-Heidelberg-Berlin, 1977.

7. H. Hironaka, Idealistic exponents of singularity, Algebraic Geometry, J. J. Sylvester Symposium, Johns Hopkins University, Baltimore, 1976, Johns Hopkins Univ. Press, Baltimore, 1977, pp. 52-125.

8. F. Kirwan, Cohomology of quotients in algebraic and symplectic geometry, Math. Notes, vol. 31, Princeton Univ. Press, Princeton, NJ, 1985.

9. __ Partial desingularisations of quotients of nonsingular varieties and their Betti numbers, Ann. of Math. (2) 122 (1985), 41-85.

10. __ Rational intersection cohomology of quotient varieties, Invent. Math. 86 (1986), 471505.

11. On spaces of maps from Riemann surfaces to Grassmannians and the cohomology of moduli spaces of vector bundles, Ark. Mat. 24 (1986), 221-275.

12. __ On the homology of compactifications of moduli spaces of vector bundles over a Riemann surface, Proc. London Math. Soc. (3) 53 (1986), 237-266.

13. J. W. Milnor and J. D. Stasheff, Characteristic classes, Ann. of Math. Stud., vol. 76, Princeton University Press, Princeton, NJ, 1974.

14. D. Mumford and J. Fogarty, Geometric invariant theory, Springer, Berlin-Heidelberg-New York, 1982.

15. A. Neeman, The topology of quotient varieties, Ann. of Math. (2) 122 (1985), 419-459.

16. P. E. Newstead, Characteristic classes of stable bundles over an algebraic curve, Trans. Amer. Math. Soc. 169 (1972), 337-345.

17. S. S. Shatz, The decomposition and specialization of algebraic families of vector bundles, Compositio Math. 35 (1977), 163-187.

18. D. Sundararaman, Moduli, deformations and classification of compact complex manifolds, Research Notes in Math, vol. 45, Pitman, Boston-London-Melbourne, 1980.

19. M. Thaddeus, Conformal field theory and the cohomology of the moduli space of stable bundles, J. Differential Geom. (to appear).

Mathematical Institute, 24-29 St. Giles, Oxford OX1 3LB, England

E-mail address: fkirwan@uk.ac.ox.vax 